\title{
Light Scattering Studies of Organic Field Effect
}

\author{
Transistors
}

A Dissertation presented to

The Faculty of the Graduate School

At the University of Missouri-Columbia

In Partial Fulfillment

of the Requirement for the Degree

Doctor of Philosophy

by

DANISH ADIL

Dr. Suchi Guha, Dissertation Supervisor

December 2013 
The undersigned, appointed by the dean of the Graduate School, have examined the dissertation entitled

\section{LIGHT SCATTERING STUDIES OF ORGANIC FIELD EFFECT TRANSISTORS}

presented by Danish Adil,

a candidate for the degree of doctor of philosophy,

and hereby certify that, in their opinion, it is worthy of acceptance.

Professor Suchi Guha

Professor Gregory Triplett

Professor Meera Chandrasekhar

Professor Gavin King 
Dedicated to my parents 


\section{ACKNOLEDGEMENTS}

I want to express my sincere gratitude to my research supervisor, Dr. Suchi Guha. I would not have been able to accomplish what I have without her exceptional support, guidance, and patience. I am indebted to her for all she has done for me and am tremendously grateful.

I would also like to thank my entire committee: Dr. Gregory Triplett, Dr. Gavin King, Dr. Meera Chandrasekhar, and Dr. Gionvanni Vignale. Their suggestions and advice were very helpful in completing this work. I also want to thank Dr. Phillip Fraundorf at the University of Missouri - St. Louis. His mentoring and guidance is deeply appreciated

I further want to thank my collaborators. Dr. Kartik Ghosh and Dr. Ram Gupta at Missouri State University made my first project possible. Dr. Satish Patil and his students were also critically important in me completing my work. I want to especially thank my colleague Ndubiusi Ukah, whose advice and company made my time here enjoyable.

I appreciate the National Science Foundation, whose generous funding made this project possible.

Lastly, I want to thank my wife, Nida, and my parents for their loving, unconditional support and encouragement. 


\section{Table of Contents}

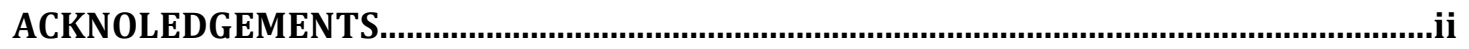

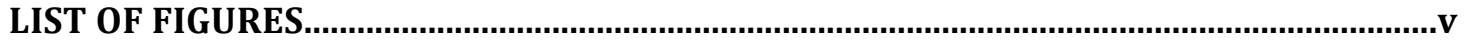

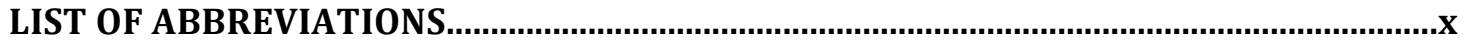

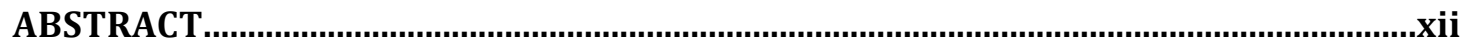

1 INTRODUCTION AND RESEARCH OBJECTIVES

2 INTRODUCTION OF ORGANIC FIELD EFFECT TRANSISTORS ..................................... 3

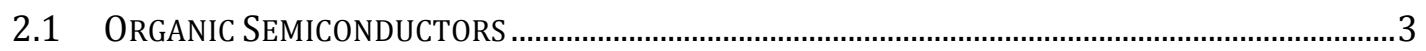

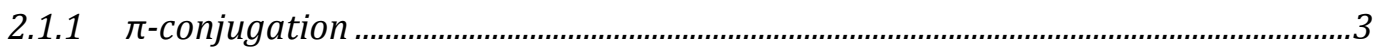

2.1.2 Organic Semiconductor Materials Used...............................................................

2.2 FIELD EFFECT TRANSISTOR THEORY ………........................................................................

3 INTRODUCTION TO INELASTIC LIGHT SCATTERING AND DENSITY FUNCTIONAL

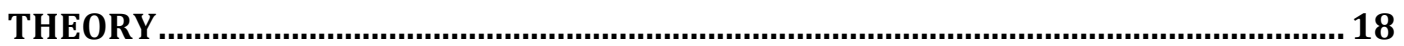

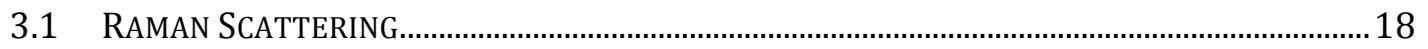

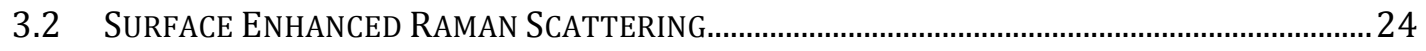

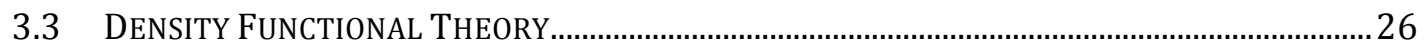

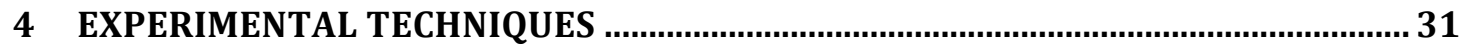

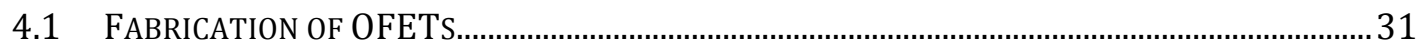

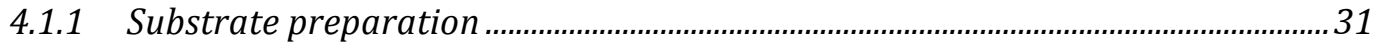

4.1.2 Film growth and Contact deposition ..........................................................................

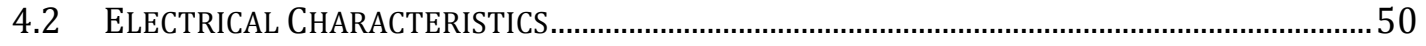




\section{PULSED LASER DEPOSITED FILMS FOR ORGANIC FIELD EFFECT}

TRANSISTORS

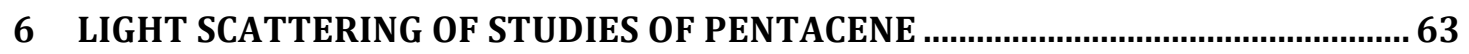

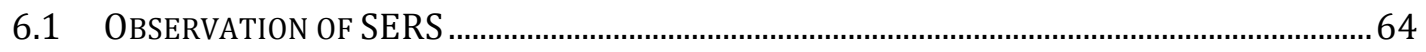

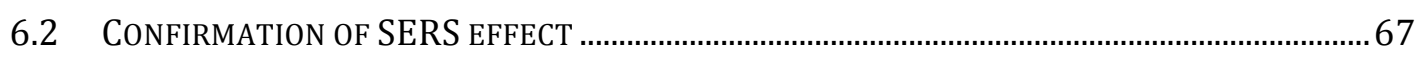

6.3 Theoretical Calculations of the Raman Spectrum of Pentacene............................... 74

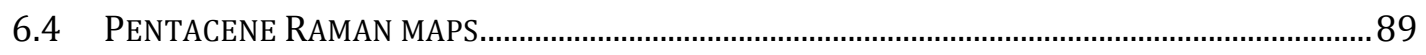

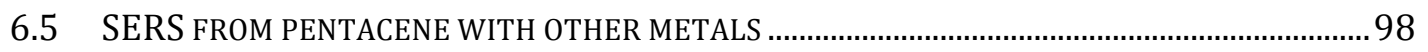

7 OFETS BASED ON SOLUTION PROCESSABLE POLYMERS ......................................

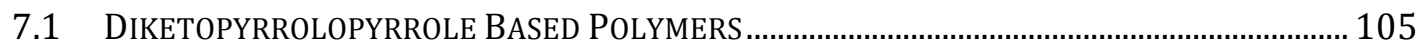

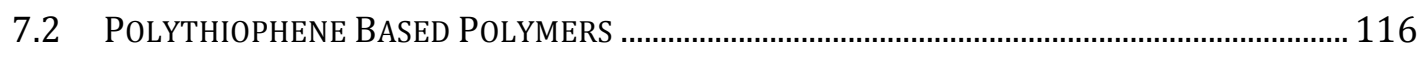

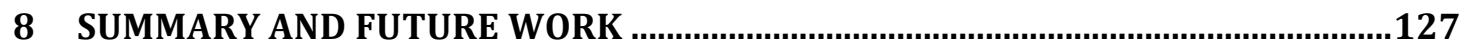

APPENDIX A: EFFECT OF OXYGEN ON TH RAMAN SPECTRUM OF PENTACENE......131 APPENDIX B: DFT CALCULATIONS OF PENTACENE HERRINGBONE

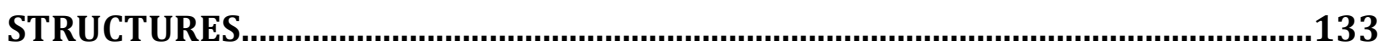

APPENDIX C: APPLYING SERS BASED DIAGNOSTIC TOOL TO PQT OFETS..................135

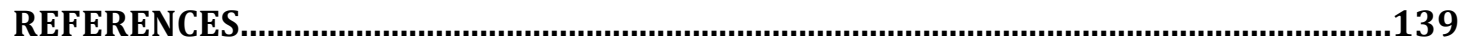

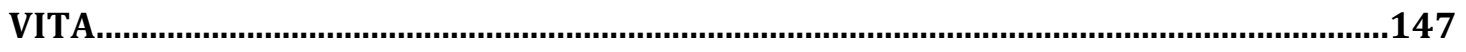




\section{LIST OF FIGURES}

2.1. The molecular structure of benzene. (a) and (b) show two ways of depicting the structure. (c) shows an alternate way that emphasizes the delocalization of electrons over the entire ring. 5

2.2. Atomic orbitals for an $\mathrm{sp}^{2}$ hybridized carbon atom shown from (a) above and (b) the side.

2.3. (a) The in phase combination of two $2 p_{z}$ orbitals form a $\pi$ orbital. This orbital, when filled, comprises a double bond. (b) the out-of-phase combination of two $2 p_{z}$ orbitals to form a $\pi^{*}$ orbital. The color depicts the phase of the orbital.............................. 7

2.4. (a) chemical structure of pentacene. (b) crystal structure of pentacene. Ref[] ........... 9

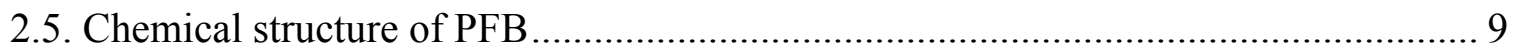

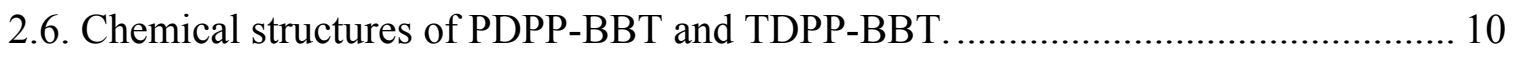

2.7. Diagram of different OFET geometries. (a) bottom-gate, top-contact; (b) bottomgate, bottom-contact; (c) top-gate, bottom-contact.......................................... 11

2.8. Diagram of a bottom gate, top contact organic field effect transistor ...................... 12

2.9. The operating regimes of an OFET. (a) linear regime. (b) pinch-off. (c) saturation regime.

2.10. Sample (a) output and (b) transfer characteristics for an OFET. In (a), six output curves corresponding to six gate voltages are shown. The saturation of $\mathrm{I}_{\mathrm{DS}}$ is clearly seen in each of the curves. In (b) both the $\mathrm{I}_{\mathrm{DS}}$ and the square root of $\mathrm{I}_{\mathrm{DS}}$ are plotted. $\mathrm{I}_{\mathrm{DS}}$ is plotted on a logarithm scale to better show the switching behavior of the OFET. The linear behavior of the squareroot of $\mathrm{I}_{\mathrm{DS}}$ is also clearly seen.

3.1. Diagram of Rayleigh and Raman scattering processes. Here $\omega_{1}$ is the angular frequency of the incident radiation and $\omega_{\text {vib }}$ is the angular frequency of the molecular vibration. 20

3.2. Two vibrations of the carbon dioxide moelecule. (a) a symmetric vibration where the dipole moment of the molecules does not change but the polarizability anisotropically. This mode is only Raman active. (b) a vibration where the dipole moment changes but the polarizability does not change anisotropically. This mode is only infrared active. 23

3.3. The SERS effect. Here $I\left(v_{L}\right)$ and $I_{\text {SERS }}\left(v_{L}\right)$ are the incident and scattered intensities, respectively. $\sigma_{\text {free }}^{R}$ and $\sigma^{R}{ }_{\text {ads. }}$ are interaction cross sections of the free and the adsorbed (via a charge transfer complex) molecules, respectively. $A\left(v_{L}\right)$ and $A\left(v_{S}\right)$ 
are the field enhancement factors are the incident and scattered frequencies,

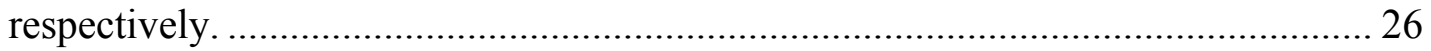

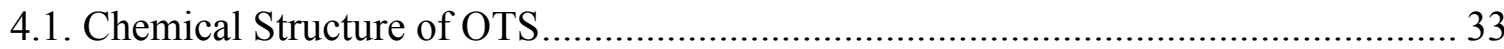

4.2 A diagram of two OTS molecules forming a portion of the OTS SAM on $\mathrm{SiO}_{2}$. Note how a water molecule is released as the OTS molecules polymerize. ...................... 34

4.3. Flow chart for $\mathrm{Si}^{++} / \mathrm{SiO}_{2}$ substrate cleaning and surface modification procedure..... 38

4.4. Schematic of the MBraun glovebox system. ........................................................ 40

4.5. Images of spun-cast semiconductor films: (a) a well formed film covering most of the substate; (b) a poorly formed film unsuitable for further use; (c) a magnified image of a heavily nucleated film................................................................. 43

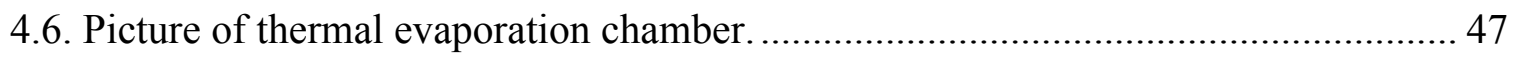

4.7. Sample mask design used for patterning top contacts for OFETs. The listed to the side of each row give the width and length of the OFET channel. The mask is $1 \mathrm{X} 1$ inch in total size. 48

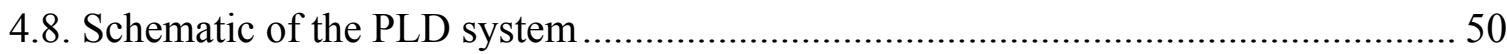

4.9. Schematic of electrical characteristics testing station......................................... 51

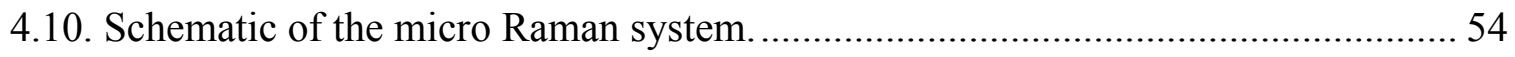

5.1. Transfer characteristics of MAPLE grown PFB-OFET on a non-OTS substrate at a drain-source voltage of $-30 \mathrm{~V}$. The inset shows the output characteristics.

5.2 Transfer characteristics of spun-cast PFB-OFET on a non-OTS substrate at a drainsource voltage of $-30 \mathrm{~V}$. The inset shows the output characteristics. 58

5.3. Transfer characteristics of MAPLE deposited PFB-OFET on an OTS treated substrate at a drain-source voltage of $-20 \mathrm{~V}$. The inset shows the output characteristics

5.4. Transfer characteristics of spun-cast PFB-OFET on an OTS treated substrate at a drain-source voltage of $-30 \mathrm{~V}$. The inset shows the output characteristics 60

5.5. Frequency dependence of the $\mathrm{C}-\mathrm{V}$ curves for (a) spun-cast $\mathrm{p}^{++}-\mathrm{Si} / \mathrm{SiO}_{2} / \mathrm{PFB}$ and (b) MAPLE grown $\mathrm{p}^{++}-\mathrm{Si} / \mathrm{SiO}_{2} / \mathrm{PFB}$ diodes. The insets show the hysteresis at $2 \mathrm{kHz}$. The arrows denote the direction of the sweep cycles.

6.1. Optical image of the channel/contact region of a pentacene-based OFET. The Raman spectrum of pentacene in the $1120-1600 \mathrm{~cm}^{-1}$ is shown on top through the Au pad (left) and from the channel region (right). 65 
6.2. The Raman spectra of pentacene films covered with Au films of three different thicknesses.

6.3. a) Schematic of the Raman setup in a backscattering geometry. (b) The Raman spectra from two regions in the sample - through a $20 \mathrm{~nm}$ thick Au layer and without any Au layer with the $785 \mathrm{~nm}$ excitation source. (c) The Raman spectra from the same regions as in (b) but measured with the $514 \mathrm{~nm}$ laser line. 69

6.4. Raman spectra of graphite through a thin Au film using the $785 \mathrm{~nm}$ and $514 \mathrm{~nm}$ excitation sources. The inset shows the Raman spectrum measured with the $785 \mathrm{~nm}$ line without any $\mathrm{Au}$. 70

6.5. The absorbance of a very thin film of Au. The absorbance signature of Au surface plasmons centered at $\sim 550 \mathrm{~nm}$ is clearly seen. 71

6.6. SEM image of the top surface of a $2 \mathrm{~nm}$ Au layer grown on top of a pentacene film. Nano-features consistent with the appearance of an SERS effect are clearly seen. An arrow is drawn at the upper left to highlight the characteristic size of the nanofeatures. The piece of dust in the lower right corner should be disregarded. 73

6.7. (a) Chemical structure of a pentacene molecule and pentacene with disordered $\mathrm{sp}^{2}$ rings. (b) Calculated Raman spectra of a pristine molecule and disordered molecule with an $\mathrm{sp}^{3} \mathrm{C}$ atom at the edge. (c) Experimental Raman spectrum of pentacene through a Au layer. The calculated spectrum is the average of the three spectra corresponding to the different schemes in (a). The dashed line correlates the experimental Raman peaks in the $1170 \mathrm{~cm}^{-1}$ region to the calculated spectrum...... 75

6.8. Optimized structures of (a) a pentacene molecule, (b) two pentacene molecules with a Au atom bonded to one of the pentacene molecules at the edge (Edge Au), and (c) $\mathrm{Au}$ atom bonded to two pentacene molecules at the center (Center $\mathrm{Au}$ ).................. 78

6.9. The HOMO (left) and LUMO (right) molecular orbitals of the Au-pentacene systems for (a) the edge Au and (b) center Au conformation ..... 80

6.10. Calculated Raman spectrum of pentacene molecules with (a) center Au and (b) edge $\mathrm{Au}$. 84

6.11. Experimental SERS spectrum of pentacene from a Au/pentacene/organic dielectric/Al OFET structure (probed through the $\mathrm{Au}$ contact). The calculated Raman spectrum is shown in red by averaging the two Au-pentacene configurations and the pristine pentacene, which is weighted as explained in the text.

6.12. The transfer plots of a pentacene OFET before and after biasing for 20 minutes at $\mathrm{V}_{\mathrm{DS}}=-30 \mathrm{~V}$ and $\mathrm{V}_{\mathrm{G}}=-40 \mathrm{~V}$. 92

6.13. The transfer plots of a PVP based pentacene OFET before and after biasing for 20 minutes at $\mathrm{V}_{\mathrm{DS}}=-3 \mathrm{~V}$ and $\mathrm{V}_{\mathrm{G}}=-3 \mathrm{~V}$. 93 
6.14. Raman linescan maps of $\mathrm{SiO}_{2}$ pentacene OFET before after bias stress. Data set 1 corresponds to the Raman spectrum measured in the channel region and data set 13 corresponds to the Raman spectrum well within the Au pad of one of the contacts 95

6.15. Raman linescan maps of a PVP based pentacene OFET before after bias stress. Data set 1 corresponds to the Raman spectrum measured in the channel region and data set 13 corresponds to the Raman spectrum well within the $\mathrm{Au}$ pad of one of the contacts

6.16. (a) The SERS spectrum of pentacene as a function of Au contact thickenss. (b) The ratio of the Raman intensity of the $1160 \mathrm{~cm}^{-1}$ peak to the $1178 \mathrm{~cm}^{-1}$ peak (measured with the $785 \mathrm{~nm}$ laser line) as a function of the thickness of the Au layer on top of the pentacene film. (c) The ratio of the Raman intensity of the $1380 \mathrm{~cm}^{-1}$ peak to the $1371 \mathrm{~cm}^{-1}$ peak (measured with the $785 \mathrm{~nm}$ laser line) as a function of the thickness of the Au layer on top of the pentacene film. 100

6.17. The SERS spectra from pentacene films with thin Ag layers evaporated on top.. 102

6.18. The Raman spectrum of a PMMA film taken both in its pristine form (No Au) and through a $\mathrm{Au}$ top contact $(\mathrm{Au})$. The SERS spectrum is clearly seen in the latter case. 104

7.1. The (a) DDP and (b) BBT subunits. Note the DPP unit here has two thiophene attached to it. 106

7.2. The experimental and theoretical absorbances of (a) PDPP and (b) TDPP 108

7.3. (a) Output and (b) transfer characteristics of PDPP-BBT OFET 110

7.4. (a) Output and (b) transfer characteristics of TDPP-BBT (sample A) OFET. 110

7.5. Transfer characteristics of TDPP-BBT (sample B) OFET. The inset shows the output characteristics from the same device

7.6. (a) Optical image of the channel/contact region in PDBB-BBT OFET. (b) Intensity profile of the $1200 \mathrm{~cm}^{-1}$ Raman peak, corresponding to the color image in (a).(c) Raman spectrum of PDPP-BBT. 114

7.7. Raman maps of PDPP-BBT along the channel/contact region before and after biasing the OFET. 115

7.8. The structures of P3HT and PQT 117

7.9. The transfer characteristics of an PQT OFET 120

7.10. The output characteristics of an PQT OFET 121 
7.11. The Raman spectrum of PQT as measure from the channel (PQT no Au) and contact (PQT Au) regions of a PQT OFET. It is apparent that the spectrum from the contact region exhibits the characteristics of SERS

7.12. The calculated Raman spectrum of pristine PQT ............................................... 123

7.13. Structure used to simulate the Raman spectrum of PQT. This structure is generic for all polythiophenes.

7.14. The structures of Au-Polythiophene disordered complexes. (a) a polythiophene subunit with a $\mathrm{Au}$ atom attached at its end (edge $\mathrm{Au}$ ) and (b) a polythiophene subunit with a $\mathrm{Au}$ atom placed at its center (center $\mathrm{Au}$ ). In both cases, the $\mathrm{Au}$ atom is $\sim 2 \AA$ from the polythiophene molecule....................................................... 125

7.15. The Raman spectra of the PQT-Au structures given in Figure 7.14 ................... 125 


\section{LIST OF ABREVIATIONS}

\begin{tabular}{|c|c|}
\hline B3LYP & Becke's three parameter hybrid \\
\hline CCD & charge coupled device \\
\hline DFT & density functional theory \\
\hline DMSO & dimethyl sulfoxide \\
\hline DPP & diketopyrrolopyrrole \\
\hline $\mathrm{ECP}$ & effective core potential \\
\hline GGA & generalized gradient approximation \\
\hline HOMO & highest occupied molecular orbital \\
\hline HOPG & highly oriented pyrolytic graphite \\
\hline LANL2DZ & Los Alamos National Laboratory 2 Double-Zeta \\
\hline LDA & local density approximation \\
\hline LUMO & lowest unoccupied molecular orbital \\
\hline$\mu$ & charge carrier mobility \\
\hline MAPLE & matrix assisted pulsed laser deposition \\
\hline OFET & organic field effect transistor \\
\hline OLED & organic light emitting diode \\
\hline OPV & organic photovoltaic \\
\hline OTS & octadecyltrichrolosilane \\
\hline P3HT & poly(3-hexylthiophene) \\
\hline PDPP-BBT & poly $\{2,6 '-4,8$-dihexyloxybenzo[1,2-b;3,4-b]dithiophene-alt-5- \\
\hline
\end{tabular}


PFB

PQT

PVP

SAM

SEM

SERS

TDPP-BBT

$\mathrm{V}_{\mathrm{Th}}$
9,9dioctylflourene-co-bis-N,N-(4-butylphenyl)-bis-N,N-phenyl-

1,4phenylenediamine)

poly-(3,3-dialkyl-quaterthiophene)

poly (4-vinyl phenol)

self-assembled monolayer

scanning Electron Microscopy

surface Enhanced Raman Spectroscopy

poly $\{2,6$ '-4,8-dihexy-loxybenzo[1,2-b;3,4-b]dithiophene-alt-5-

dihexyl-3,6-bis(5-thiophen-2-yl)pyrrolo[3,4-c]pyrrole-1,4-dione

threshold voltage 


\begin{abstract}
Organic semiconductors hold a great promise of enabling new technology based on low cost and flexible electronic devices. While much work has been done in the field of organic semiconductors, the field is still quite immature when compared to that of traditional inorganic based devices. More work is required before the full potential of organic field effect transistors (OFETs), organic light emitting diodes (OLEDs), and organic photovoltaics (OPVs) is realized. Among such work, a further development of diagnostic tools that characterize charge transport and device robustness more efficiently is required. Charge transport in organic semiconductors is limited by the nature of the metal-semiconductor interfaces where charge is injected into the semiconductor film and the semiconductor-dielectric interface where the charge is accumulated and transported. This, combined with that fact that organic semiconductors are especially susceptible to having structural defects induced via oxidation, charge transport induced damage, and metallization results in a situation where a semiconductor film's ability to conduct charge can degrade over time. This degradation manifests itself in the electrical device characteristics of organic based electronic devices. OFETs, for example, may display changes in threshold voltage, lowering of charge carrier mobilities, or a decrease in the On/Off ratio. All these effects sum together to result in degradation in device performance.
\end{abstract}

The work begins with a study where matrix assisted pulsed laser deposition (MAPLE), an alternative organic semiconductor thin film deposition method, is used to fabricate OFETs with improved semiconductor-dielectric interfaces. MAPLE allows for the 
controlled layer-by-layer growth of the semiconductor film. Devices fabricated using this technique are shown to exhibit desirable characteristics that are otherwise only achievable with additional surface treatments. MAPLE is shown to be viable alternative to other fabrication methods.

The work continues with a combined electro-optical study of the metal-semiconductor interface in OFETs. It is highly desirable that a method that can be used to understand the mechanisms of device performance degradation be developed. We demonstrate that the surface enhanced Raman (SERS) effect (at the metal-semiconductor interface) can serve as such a method. We first show how the Raman spectrum of a pristine pentacene (a common organic semiconductor) film is dramatically different from the spectrum collected when the film is probed through a metal contact. The spectrum collected from the contact region exhibits a change in peak intensities, peak positions, and an overall enhancement of signal intensity, all of which are direct evidence of the SERS effect. The SERS spectrum is then modeled by first principles density functional theory (DFT). The DFT calculations demonstrate that the SERS effect shows an extreme sensitivity to disorder in these semiconductor films.

We further show how the SERS spectrum evolves after the device has been subjected to a bias-stress (i.e. applying both gate and drain voltages for an extended period of time). Devices that exhibit a strong degradation in performance also feature a concurrent change of the SERS spectrum. On the other hand, we see no change in the SERS spectrum of devices that exhibit stable operating characteristics. Thus, we confirm that the SERS spectrum can be used as a diagnostic tool for correlating transport properties to structural changes, if any, in organic semiconductor films. In conclusion, we develop a non- 
invasive opto-electronic visualization tool that can be used as an in-situ probe to characterize charge transport in organic semiconductor devices. 


\section{Introduction and Research Objectives}

Since the first report of a highly conducting organic polymer, polyacetylene, in 1977 by A.J. Heeger, H. Shirakawa, and Alan G. MacDiamid (Nobel Prize in Chemistry in $2000)^{1}$, the field of organic electronics has grown significantly. Organic electronics are electronic devices built using organic semiconductors. These organic semiconductors are typically heavily conjugated small molecules or polymers. The heavy conjugation leads to a delocalization of $\pi$-orbitals, which then serve as the path for electrical conduction. The physics of charge carrier conduction for these organic semiconductors is quite different than traditional inorganic semiconductors. Nonetheless, parallels can be drawn and thus, the device physics, in many ways, for organic devices correlate well with the device physics for traditional devices.

Organic semiconductors hold a great promise of enabling new technology based on low cost and flexible electronic devices. While much work has been done in the field organic semiconductors, the field is still quite immature when compared to that of traditional inorganic based devices. Further work is required before the full potential of organic field effect transistor (OFETs), organic light emitting diodes (OLEDs), and organic photovoltaics (OPVs) is realized. Among such work, a further development of diagnostic tools that characterize charge transport and device robustness more efficiently is required.

This report starts with an overview of the experimental and theoretical methods used in the work. Chapter 2 reviews the theoretical background of organic semiconductors and OFETs. Chapter 3 discusses the experimental methods used in this work. The dissertation 
continues in chapter 4 with an investigation of an alternative (to commonly used methods) method of semiconductor film deposition and its implications to the dielectricsemiconductor interface. Chapter 6 details a study in which Raman scattering is used to develop a new method of diagnosing disorder at the metal-semiconductor interface in OFET fabricated using a vacuum evaporated conjugated molecule. The method is also shown to serve as tool to understand the origin of device performance degradation over time. In chapter 7 , the method developed in the previous chapter is used on OFETs fabricated with solution processable polymers, including first OFETs fabricated from a series of new diketopyrrolopyrrole based organic semiconductors. These materials are shown to be particularly robust and to resist the degradation mechanisms seen in chapter 6. Finally, chapter 8 contains a brief summary and suggestions for future work. 


\section{Introduction of Organic Field Effect Transistors}

\subsection{Organic Semiconductors}

Organic semiconductors are essentially plastics that can be made to conduct. Being plastics, these materials generally from soft, flexible, and transparent films. Organic semiconductors are typically carbon-based materials that feature a backbone formed by the joining of $\pi$-conjugated, unsaturated moieties. ${ }^{2}$ This results in materials that feature highly conjugated and delocalized $\pi$ electrons. These materials may be small molecules or polymers, and further may form amorphous or semi-crystalline films. ${ }^{3}$ However, in either case, the film should consist of well-packed molecules thus ensuring 2D electron transport within molecular layers. ${ }^{4}$

Charge transport in organic semiconductors is fundamentally different than charge transport in inorganic semiconductors. Unlike the band transport that is seen in inorganic semiconductors, charge transport in organic semiconductors proceeds via a thermally assisted hopping mechanism via a Gaussian distribution of electronic states. ${ }^{5}$ Evidence of this is seen in the temperature dependence of charge carrier mobility. ${ }^{6}$ Thus, unlike inorganic semiconductors where conductivity is determined by crystal structure, charge transport in organic semiconductors is critically dependent on the packing of chains and the density of structural defects. ${ }^{3}$

\subsection{1 $\pi$-conjugation}

Most organic compounds are insulators. Conjugated $\pi$-bonds are what give organic semiconductors the unique ability to conduct charge. These materials have alternating carbon-carbon double and single bonds. Due to their conjugation, it is generally possible 
to draw the molecular structure of these compounds in more than one way. Specifically, the double bonds and single bonds can be interchanged. This phenomenon, known as resonance, shows that the electrons comprising the double bonds (the $\pi$-electrons) are not localized to specific bonds, but are, in fact, delocalized and may be assigned to more than one constituent atom. Thus, these $\pi$-electrons constitute a continuous electron density in the periodic potential of the carbon nuclei. Now, one may naively think of this material as a one-dimensional metal, as the $\pi$-electrons (one per carbon atom) partially fill the highest occupied band. However, such a one-dimensional metal is unstable, and undergoes a transition to a dimerized state. ${ }^{7}$ This opens a bandgap, giving the material its semiconducting properties.

A special case of $\pi$-conjugation occurs in planar ring systems where the delocalization is especially pronounced. These systems are said to be aromatic. ${ }^{8}$ Such aromatic rings form the backbones of most commonly used organic semiconducting materials. Figure 2.1 gives the molecular diagrams of the simplest aromatic ring, benzene. Figure 2.1 (c) demonstrates an alternative means of depicting the delocalized $\pi$ electrons. This emphasizes how they are delocalized over the entire ring. 


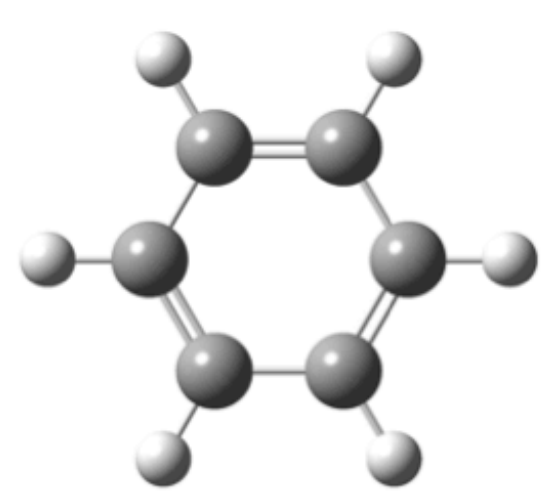

(a)

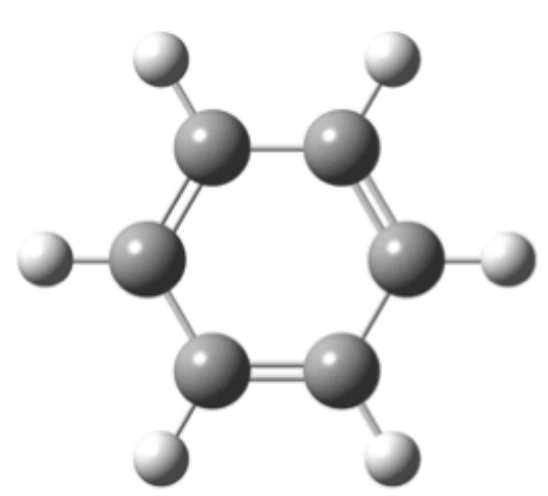

(b)

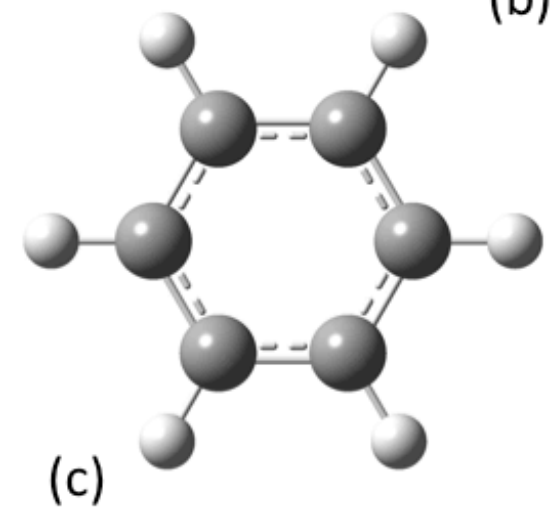

Figure 2.1. The molecular structure of benzene. (a) and (b) show two ways of depicting the structure. (c) shows an alternate way that emphasizes the delocalization of electrons over the entire ring.

The carbon atoms in conjugated systems are said to be $\mathrm{sp}^{2}$ hybridized. That is to say, the $2 \mathrm{~s}$ orbital has linearly combined with the $2 \mathrm{p}_{\mathrm{y}}$ and the $2 \mathrm{p}_{\mathrm{z}}$ orbitals to create three degenerate $\mathrm{sp}^{2}$ orbitals. The last $2 \mathrm{p}_{\mathrm{z}}$ orbital remains unaffected. Figure 2.2 shows the shape of the atomic orbitals for a carbon atom from the (a) top and (b) side. The $\mathrm{sp}^{2}$ orbitals, shown in red, are separated by $120^{\circ}$. The $2 \mathrm{p}_{z}$ orbitals, shown in green, are offset $90^{0}$ from the plane formed by the $\mathrm{sp}^{2}$ orbitals. 


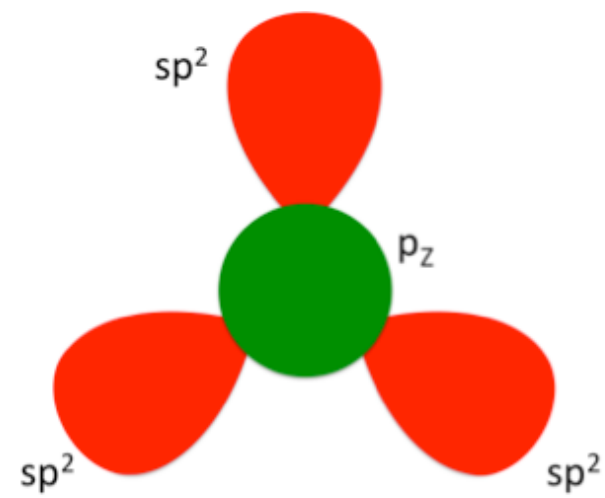

(a) Top View

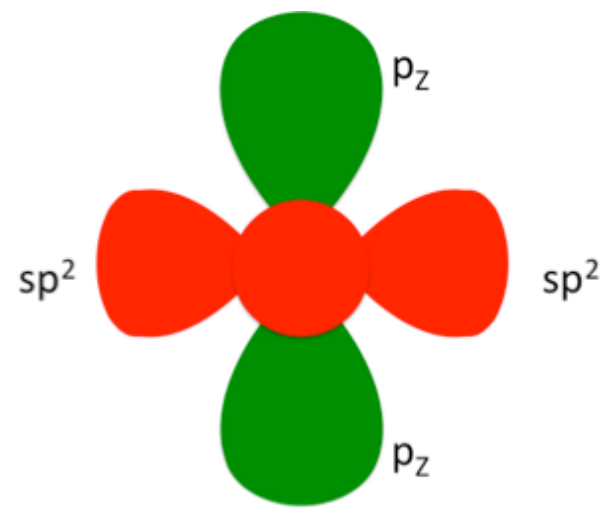

(b) Side View

Figure 2.2. Atomic orbitals for an $\mathrm{sp}^{2}$ hybridized carbon atom shown from (a) above and (b) the side.

The three $\mathrm{sp}^{2}$ orbitals in an $\mathrm{sp}^{2}$ hybridized atom form the single bonds and $2 \mathrm{p}_{\mathrm{z}}$ orbital forms the double bonds. Thus, a carbon atom in a conjugated system will typically be bonded to three other atoms, which form a plane and are separated by $120^{\circ}$. In addition, it will be double bonded to one of these other atoms. An example of two $\mathrm{sp}^{2}$ hybridized carbon atoms forming a double bond in an ethylene molecule is shown in Figure 2.3. Figure 2.3 (a) shows how the $2 \mathrm{p}_{\mathrm{z}}$ orbitals combine in phase to form the $\pi$ orbital. Each carbon atom contributes one electron to the $\pi$ orbital. The two electrons that comprise the orbital are then shared equally between the two atoms. It is important to note that the $\pi$ orbital is the highest lying occupied molecular orbital in this system. The next highest orbital (the first unoccupied orbital) is formed by the out of phase combination of the $2 \mathrm{p}_{z}$ orbitals. It is referred to as the $\pi^{*}$ anti-bonding orbital. It is shown in Figure 2.3 (b). If an electron is added to molecule, it will reside in this orbital. Thus, the hybridization of individual $2 \mathrm{p}_{\mathrm{z}}$ atomic orbitals yield $\pi$ and $\pi *$ molecular orbitals 


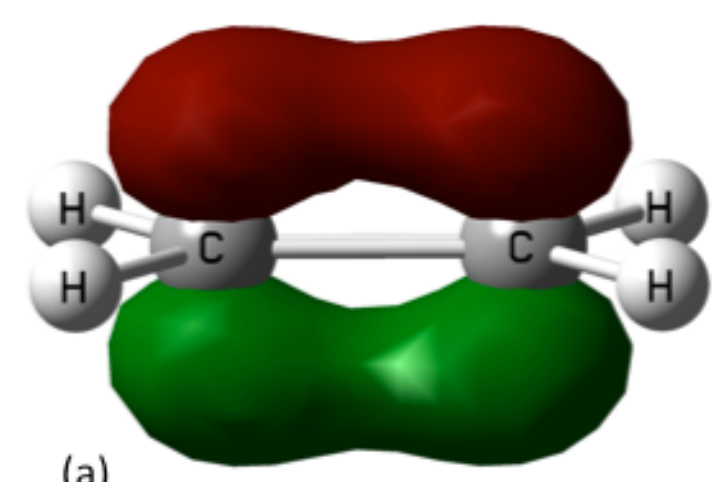

(a)

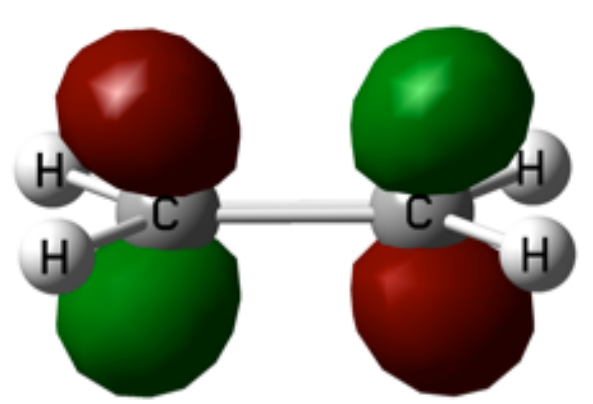

(b)

Figure 2.3. (a) The in phase combination of two $2 p_{z}$ orbitals form a $\pi$ orbital. This orbital, when filled, comprises a double bond. (b) the out-of-phase combination of two $2 p_{z}$ orbitals to form a $\pi^{*}$ orbital. The color depicts the phase of the orbital.

In the case of large, highly conjugated molecules and polymers, the $\pi$ and $\pi^{*}$ orbitals are delocalized over the entire backbone of the molecule. These orbitals are labeled as the highest occupied molecular orbital (HOMO) and the lowest unoccupied molecular orbital (LUMO), respectively. These orbitals may be separated by only a few electron volts. These two orbitals act in a similar manner to the valence and conduction bands in traditional inorganic semiconductors. Thus, for the purposes of understanding devices such as OFETs, one can start with the well-studied and understood inorganic device theory.

\subsubsection{Organic Semiconductor Materials Used}

The work described in this thesis primarily makes use of the three different semiconducting materials shown in Figure 2.4-Figure 2.6. Figure 2.4 shows the chemical structure of pentacene, a commonly used small molecule. Being a small molecule, it is typically thermally evaporated. It forms polycrystalline films in the herringbone structure 
as seen in Figure 2.4 (b). ${ }^{9}$ Grazing incidence x-ray spectroscopy has been used to show that the film is formed via the growth of single crystal islands on the substrate surface. ${ }^{10}$ The islands may be dendritic or faceted, depending on the substrate used, with faceted islands giving higher mobilities. ${ }^{11}$ The average crystal size within the film is also dependent on substrate temperature during deposition with elevated temperatures resulting in larger crystals. ${ }^{12}$ Thus, it follows that elevated substrate temperatures result in higher mobilities. $^{13}$

Figure 2.5 shows the chemical structure of the fluorene co-polymer (9,9dioctylflourene-co-bis-N,N-(4-butylphenyl)-bis-N,N-phenyl-1,4phenylenediamine)

(PFB). PFB is solution processable and serves as a good test system for other polymer semiconductors. Finally, the structure of two diketopyrrolopyrrole based polymers: Poly \{2,6'-4,8-dihexyloxybenzo[1,2-b;3,4-b]dithiophene-alt-5-dihexyl-3,6-bis-(4phenyl)pyrrolo[3,4-c]pyrrole-1,4-dione $\}$ (PDPP-BBT) and Poly $\{2,6$ '-4,8-dihexyloxybenzo[1,2-b;3,4-b]dithiophene-alt-5-dihexyl-3,6-bis(5-thiophen-2-yl)pyrrolo[3,4c]pyrrole-1,4-dione\} (TDPP-BBT) are given in Figure 2.6. Diketopyrrolopyrrole (DPP) based low bandgap materials are not just useful for device applications but are designed to achieve environmentally stable organic semiconductors. The strong intermolecular interaction leads to molecular packing with a large electronic bandwidth, thus improving the charge- transport properties. Specifically, the interaction of hydrogen atoms from the aromatic rings and oxygen atoms from the carbonyl groups cause an alignment of the polymer backbones. Of the two materials, TDPP is more planar than PDPP. The increased torsion angle in PDPP is likely due to a greater steric interaction of the phenyl 
hydrogen atoms with the carbonyl oxygen atom. The hydrogen atoms on the smaller thiophene rings in TDPP have less of a steric interaction.

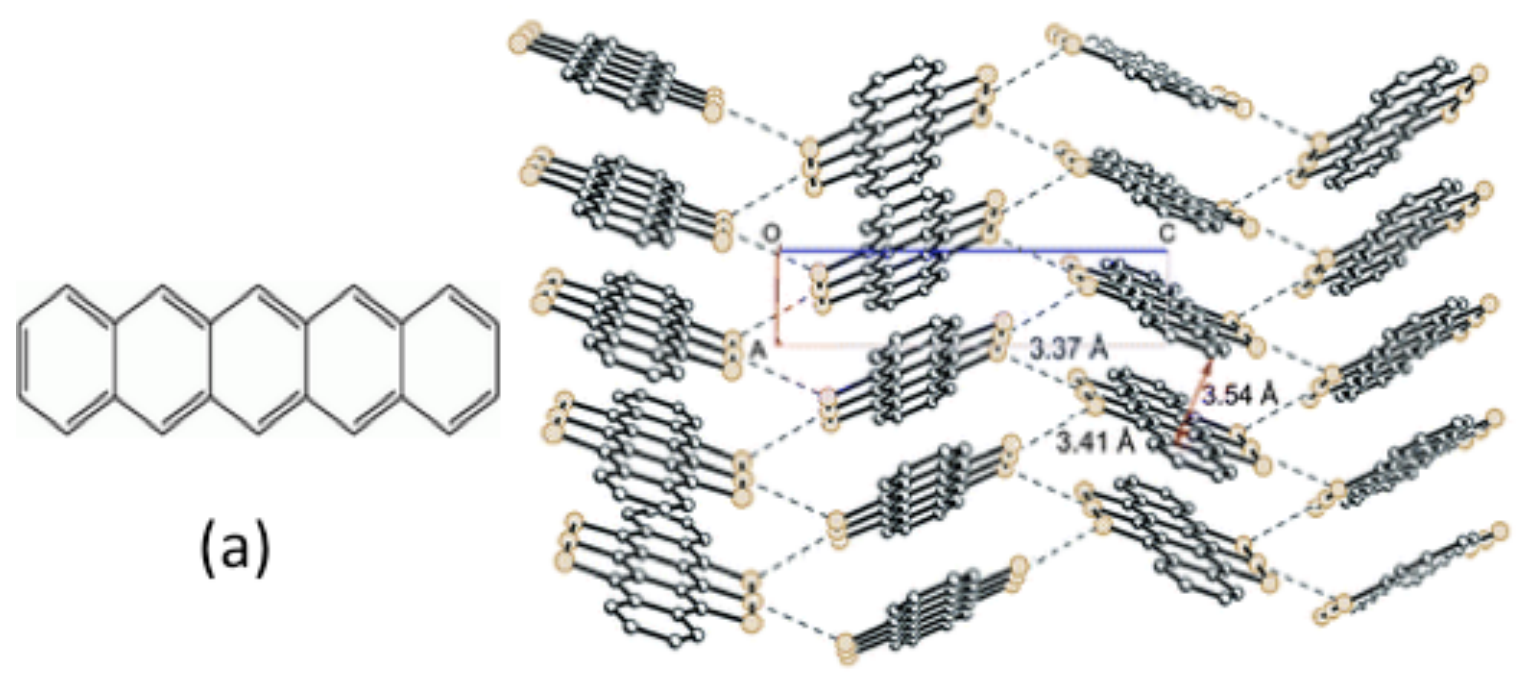

(b)

Figure 2.4. (a) chemical structure of pentacene. (b) crystal structure of pentacene. $\operatorname{Ref[14]}$

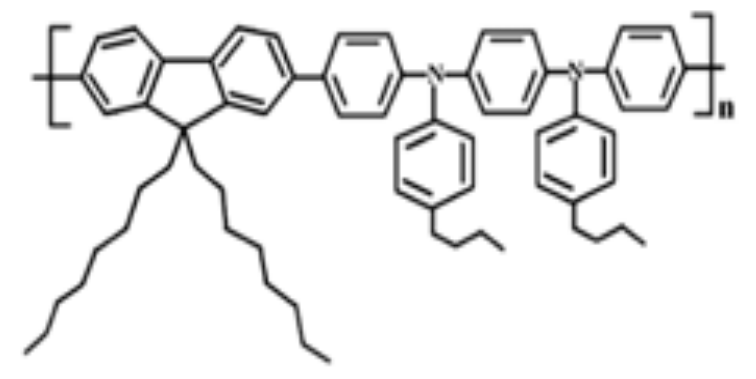

Figure 2.5. Chemical structure of PFB 


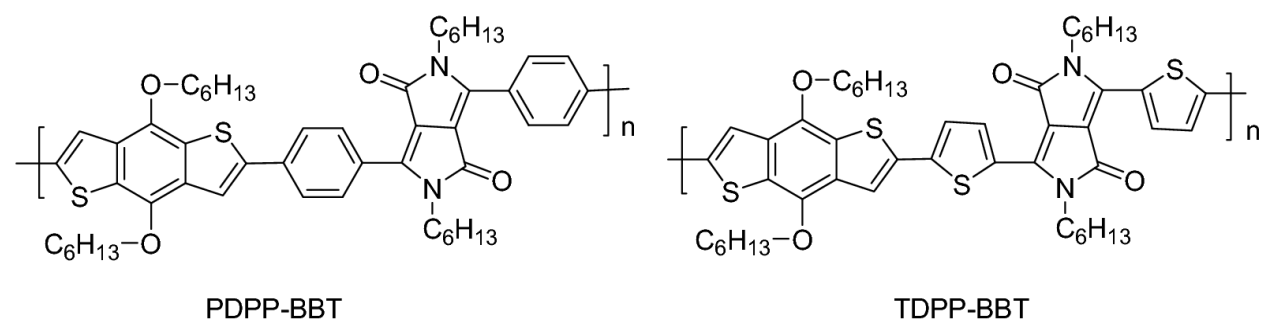

Figure 2.6. Chemical structures of PDPP-BBT and TDPP-BBT.

\subsection{Field Effect Transistor Theory}

A transistor is a three terminal device where a potential applied to one terminal results in the formation of a conductive channel between the other two. Thus, conduction between the latter two terminals is gated by the first terminal, resulting in a situation where the ideal transistor can be in one of two states: on, where the transistor is able to conduct current; and off, where the transistor does not conduct current. In a field effect transistor, a potential applied to the gating terminal induces, via an electric field through a dielectric film, the accumulation of charge in a semiconducting layer. This accumulated, mobile charge then acts as the conductive channel between the former two electrodes. In an organic field effect transistor (OFET), the semiconducting layer is comprised of a thin film of an organic semiconductor.

In field effect transistors, the gating electrode is referred to as the gate, while the other two electrodes are referred as the source and drain. The gate electrode must be separated from the semiconducting layer by a dielectric. The source and drain electrodes must be in direct contact with the semiconductor, as they will have to inject and extract charge carriers, respectively. The gate electrode (along with its dielectric) may be placed above or below the semiconductor film. Based on the placement of the gate, the device may be 
classified as either top-gate or bottom-gate. Likewise, devices may also be classified as top-contact or bottom contact, depending on the location of the source and drain electrodes relative to the semiconductor film. A diagram of the various types of geometries is show in Figure 2.7 All devices described in this work were of the bottomgate, top-contact variety. Regardless of the geometry used, the metal-semiconductor interface, where charge is injected into the semiconductor, and the semiconductordielectric interface, along which charge is accumulated, both are critically important to device performance.

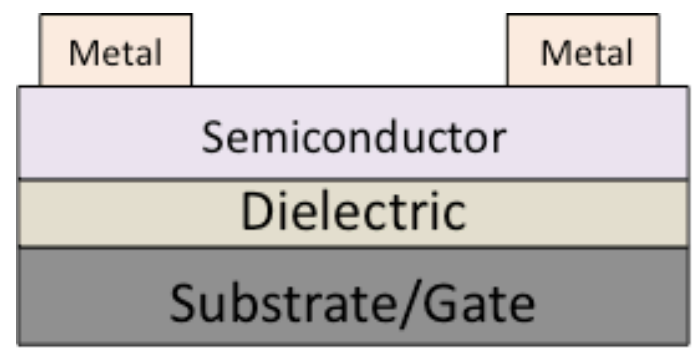

(a)

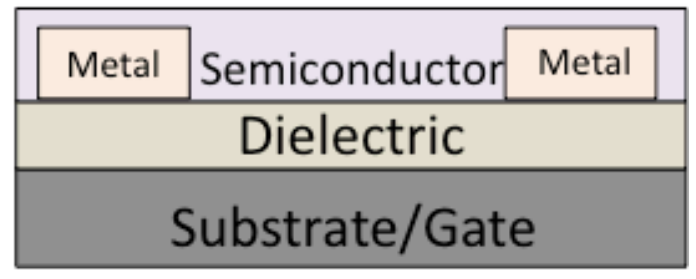

(b)

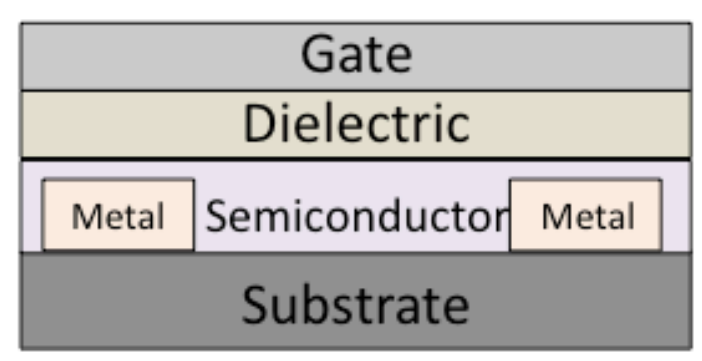

\section{(c)}

Figure 2.7. Diagram of different OFET geometries. (a) bottom-gate, top-contact; (b) bottom-gate, bottom-contact; (c) top-gate, bottom-contact. 
A schematic of a typical bottom gate, top gate OFET used in this work is shown in Figure 2.8. Here, voltage applied to the gate terminal $\left(\mathrm{V}_{\mathrm{G}}\right)$ gates conductivity between the other two terminals (source and drain) via an electric field through the dielectric layer. The source and drain contacts have a width $\mathrm{W}$ and are separated by a distance L. Once the device has been switched on via $\mathrm{V}_{\mathrm{G}}$, voltage applied to the drain terminal $\left(\mathrm{V}_{\mathrm{DS}}\right)$ creates a drain current IDS. The source contact is typically grounded.

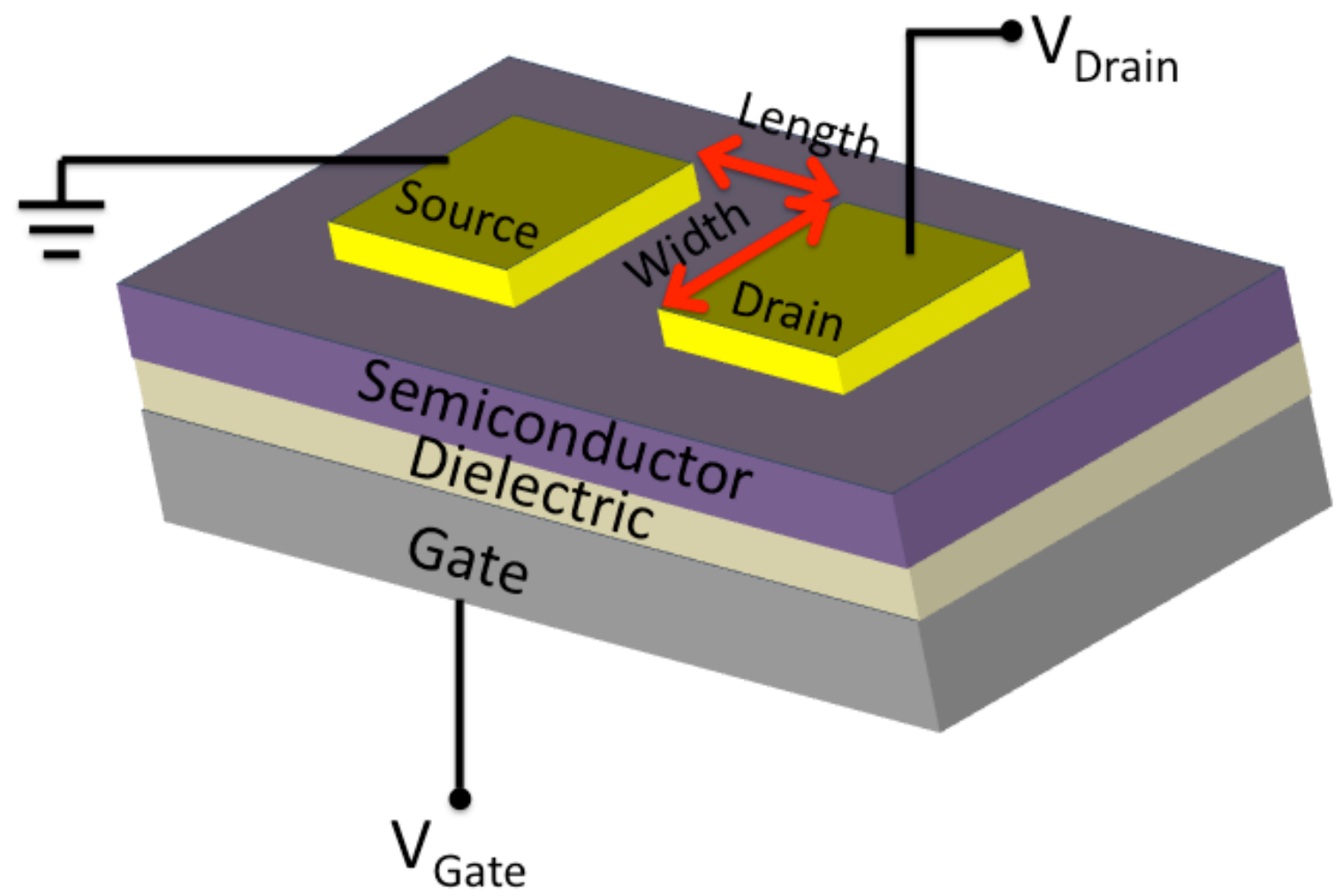

Figure 2.8. Diagram of a bottom gate, top contact organic field effect transistor

We use the long channel approximation for the OFETs where the horizontal electric field between the drain and the source is assumed to be small compared to the vertical electric field induced by the gate. When a gate voltage is applied, a charge proportional to the capacitance of the dielectric is induced at the dielectric-semiconductor 
interface. Not all the charge is mobile though, as traps within the semiconducting layer must first be filled before mobile charge can begin accumulating. The gate voltage at which charge begins accumulating is referred to as the threshold voltage $\left(\mathrm{V}_{\mathrm{Th}}\right)$. The amount of charge along the channel varies linearly as function of position due to the drain-source bias:

$$
Q_{i}(x, y)=C_{0}\left[V_{G}-V_{T h}-V(x)\right],
$$

where $\mathrm{x}$ and $\mathrm{y}$ are taken to be along the length and width of the channel respectively. Here, $\mathrm{V}(\mathrm{x})$ varies linearly from the source $(\mathrm{x}=0, \mathrm{~V}(\mathrm{x})=0)$ to the drain $(\mathrm{x}=\mathrm{L}, \mathrm{V}(\mathrm{x})=$ $\mathrm{V}_{\mathrm{DS}}$ ). Initially, increasing $\mathrm{V}_{\mathrm{DS}}$ results in a greater amount of charge being accumulated and thus results in an increase in $\mathrm{I}_{\mathrm{DS}}$. The device is said to be in the linear regime. If $\mathrm{V}_{\mathrm{DS}}$ is further increased such that $V_{D S}=V_{G}-V_{T h}$, the channel is "pinched off" and a further increase in $V_{D S}$ will not result in an increase in $I_{D S}$. In this case, the vertical electric field in the channel close to the drain is insufficient to cause the accumulation of charge. IDS is said to be saturated in this case. Figure 2.9 shows the operating regimes of an OFET. ${ }^{15}$ 

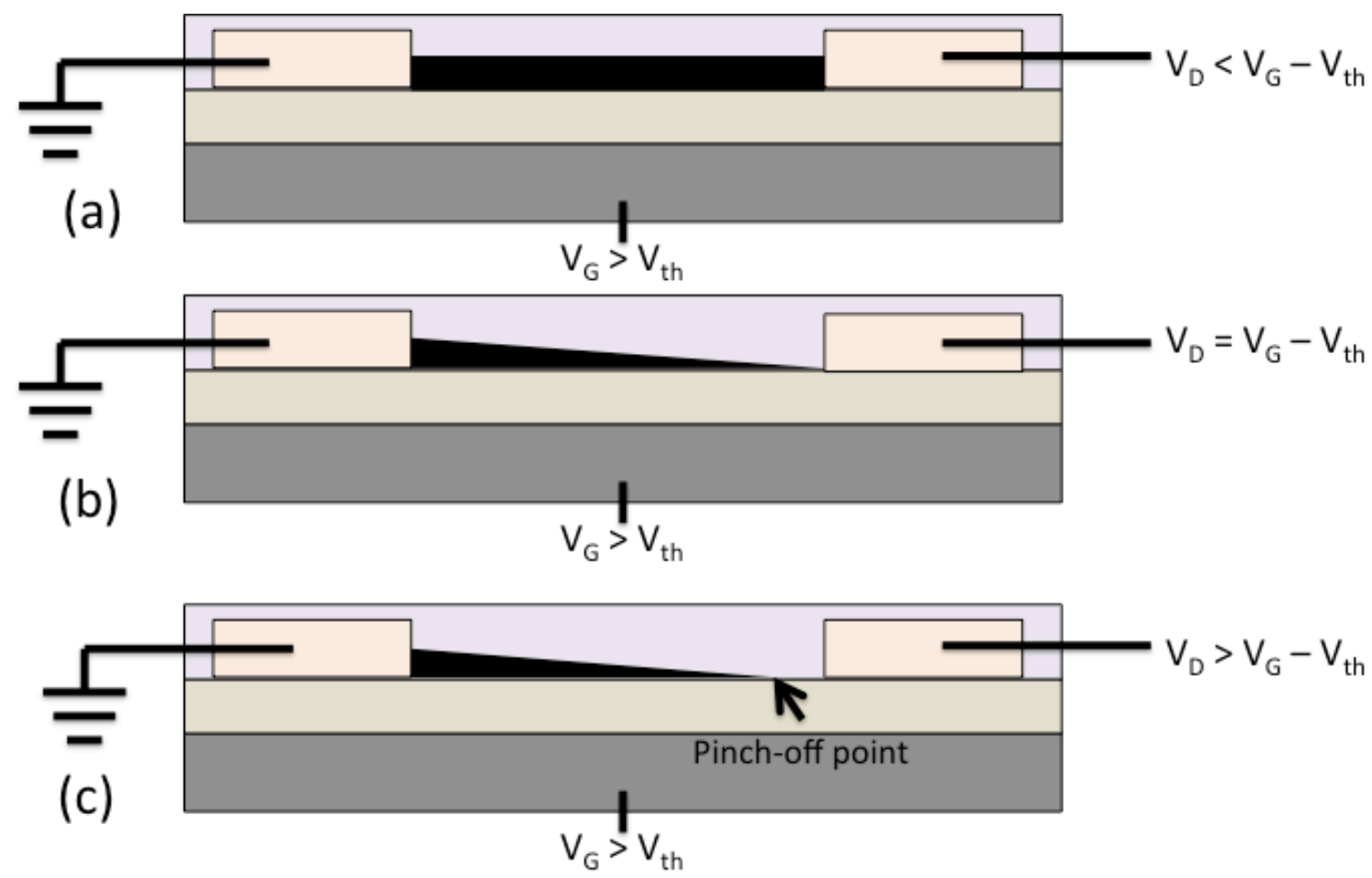

Figure 2.9. The operating regimes of an OFET. (a) linear regime. (b) pinch-off. (c) saturation regime.

Inserting the charge distribution given in equation 2.1 into the well known drift current equation gives:

$$
i(x, y)=Q_{i}(x, y) \mu \frac{d V}{d x}
$$

where $i(x, y)$ is the linear current density $(\mathrm{Amp} / \mathrm{cm})$ in the channel. Since the current is completely uniform along the width of the channel, one may quickly integrate over the $y$ coordinate to get

$$
I_{D S}(x)=W Q_{i}(x, y) \mu \frac{d V}{d x}
$$

where $I_{D S}(x)$ is the current (Amps) in the channel as a function of the position along the length of the channel. Equation 2.3 can be rearranged to give 


$$
\int_{0}^{L} I_{D S}(x) d x=\mu W \int_{0}^{V_{D S}} Q_{i}(x) d V .
$$

Here, the integration is taken to be over the channel length L. A change of variables is used to express the right hand side as a function of voltage $\left(\mathrm{x}=0 \rightarrow \mathrm{V}=0, \mathrm{x}=L \rightarrow \mathrm{V}=V_{D S}\right)$. Inserting the equation 2.1 into equation 2.4 gives

$$
\int_{0}^{L} I_{D S}(x) d x=\mu W \int_{0}^{V_{D S}} C\left[V_{G}-V_{T h}-V(x)\right] d V .
$$

Finally, one can integrate to get the expression for total current in the OFET:

$$
I_{D S}=\frac{\mu W}{L} C\left[\left(V_{G}-V_{T h}\right) V_{D S}-\frac{V_{D S}^{2}}{2}\right] .
$$

In the saturation regime, where $V_{D S}=V_{G}-V_{T h}$ and $V_{G} \gg V_{T h}$, The total current takes the form

$$
I_{D S}=\frac{\mu W C_{0}}{2 L}\left(V_{G}-V_{T h}\right)^{2}
$$

Equation 2.7 can be rearranged to give an expression for the saturation mobility, $\mu$ :

$$
\mu=\frac{2 L}{W C_{0}}\left(\frac{\partial \sqrt{I_{D S}}}{\partial V_{G}}\right)^{2}
$$

Thus, if the electrical characteristics of an OFET can be measured, then they can be fit to the above relations, allowing for the extraction of device parameters such as $\mu$ and $\mathrm{V}_{\mathrm{Th}}$. To properly characterize the OFET, we must keep in mind that there are two voltages, $V_{G}$ and $V_{D S}$, that may be sourced, thus giving two currents, $I_{G S}$ and $I_{D S}$, that may be measured. The current $\mathrm{I}_{\mathrm{GS}}$ is the leakage current though the gate dielectric. Ideally, it would be zero, but as long as it is much, much smaller than the transistor current $\mathrm{I}_{\mathrm{DS}}$, it may be disregarded. This leaves us the current $\mathrm{I}_{\mathrm{DS}}$, which we would like to characterize as a function of the input voltages $\mathrm{V}_{\mathrm{G}}$ and $\mathrm{V}_{\mathrm{DS}}$. 
The output characteristics of an OFET are behavior of $I_{D S}$ as a function of $V_{D S}$ at various gate voltages. As described above, for a given gate voltage, the $I_{D S}$ will increase linearly with $V_{D s}$ until it becomes saturated. As $V_{G}$ in incremented to a higher (in magnitude) value, the saturation point will move to a higher value and the saturation current will increase as well. A sample set of output curves is show in Figure 2.10 (a)

The transfer characteristics of an OFET is the behavior of $\mathrm{I}_{\mathrm{DS}}$ as a function of $\mathrm{V}_{\mathrm{G}}$. The transfer curve will be measured for a chosen value of $V_{D S}$ known as the operating voltage. A sample transfer curve is shown in Figure 2.10 (b). Here, the switching behavior of the transistor is clearly seen. $\mathrm{I}_{\mathrm{DS}}$ increases quadratically with $\mathrm{V}_{\mathrm{G}}$, quickly taking the transistor from an off state to an on state. It is seen from equation 2.2 that the square root of $I_{D S}$ should be linear with $V_{G}$ in the saturation regime. To confirm this, the square root of $I_{D S}$ is also often plotted when plotting transfer characteristics, as it is in Figure 2.10 (b). A linear fit to this region allows for the calculation of $\mu$ by using equation 2.3. $\mathrm{V}_{\mathrm{Th}}$ is then computed by extrapolating the linear fit to the $\mathrm{x}$-axis. Lastly, the On/Off ratio can also be easily computed from the transfer data. The On/Off ratio is the ratio of the on current, defined to be $\mathrm{I}_{\mathrm{DS}}$ when $\mathrm{V}_{\mathrm{G}}$ is equal to the device operating voltage, to the off current, defined either as the minimum value Ids takes or alternatively the value of $I_{D S}$ when $V_{G}$ is set to its "off" value. 

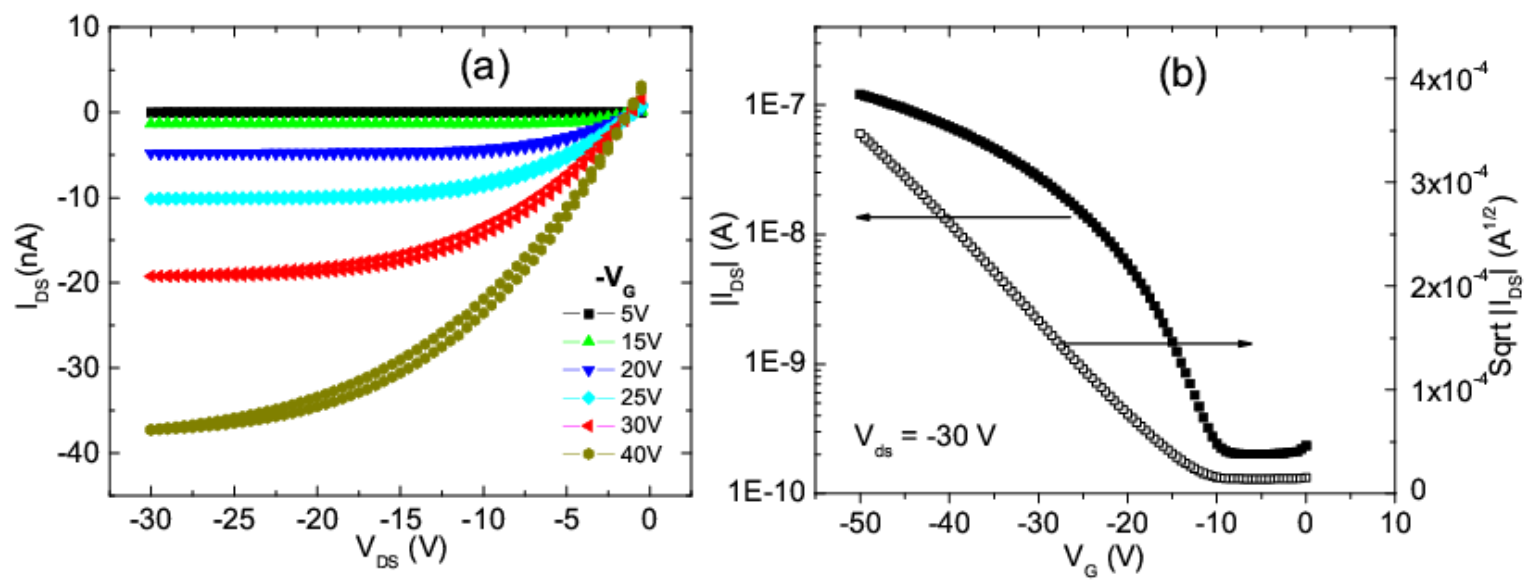

Figure 2.10. Sample (a) output and (b) transfer characteristics for an OFET. In (a), six output curves corresponding to six gate voltages are shown. The saturation of $\mathrm{I}_{\mathrm{DS}}$ is clearly seen in each of the curves. In (b) both the $\mathrm{I}_{\mathrm{DS}}$ and the square root of $\mathrm{I}_{\mathrm{DS}}$ are plotted. $\mathrm{I}_{\mathrm{DS}}$ is plotted on a logarithm scale to better show the switching behavior of the OFET. The linear behavior of the squareroot of $\mathrm{I}_{\mathrm{DS}}$ is also clearly seen. 


\section{Introduction to Inelastic Light Scattering and Density Functional Theory}

\subsection{Raman Scattering}

When light is incident on a material, it may be absorbed, transmitted, or scattered. While the absorbance of a material clearly serves as a source for essential information about its electronic structure, the scattered light may also contain fundamental information about the material. The vast majority of the scattered light is scattered elastically, following the well-known $\omega^{4}$ function first derived by Lord Rayleigh, as described in [16]. In 1928, it was discovered by Sir C. V. Raman, however, that not all of the incident light is scattered elastically. ${ }^{17}$ Raman irradiated liquid samples by sunlight passed through a violet filter. The scattered light was subsequently passed through a green filter. While the understanding at that time would suggest that all scattered light would be the same color as the incident light, Raman showed that, in fact, some of the scattered light had changed color. This inelastic scattering phenomenon was named the Raman effect. Raman won the Nobel Prize in physics in 1930 for his discovery.

In the Raman effect, the incident photon loses or gains energy from a quasiexcitation in the analyte. These excitations may be discrete vibrations, phonons, or plasmons. ${ }^{18}$ The change in the photon energy is equal to the energy of the excitation with which the photon interacted. Thus, the spectrum of the inelastically scattered light will contain information about the energies of the molecular vibrations of the analyte, for example. 
The Raman effect is very weak, as only about 1 in $10^{7}$ photons is inelastically scattered, so isolating this spectrum from the Rayleigh scattered light is generally non-trivial.

When a photon is incident on a molecule, it can perturb the molecule to an excited virtual state via its electric field. When the molecule relaxes back (re-emitting a photon) to its ground electronic state, it may fall to a different vibrational state. A photon that induces a transition where the net result is a transition from the ground vibrational state to a higher vibrational state is said to have undergone a Stokes shift. Whereas a photon that induces a transition where the net result is a transition from a higher vibrational state to the ground state is said to have anti-Stokes shifted. A diagram of the above scattering processes is given in Figure 3.1. It should be noted that while the Stokes and anti-Stokes shifted photons will be shifted by energies of equal magnitude (the Stokes shifted photons will have lost this amount of energy while the anti-Stokes will have gained energy, of course), the relative intensities of the two peak in the Raman spectrum will not be of equal magnitude. The Stokes shifted peak will generally be much stronger than the anti-Stokes peak on account of the higher thermal occupation number of the ground vibrational state over the first excited state. 


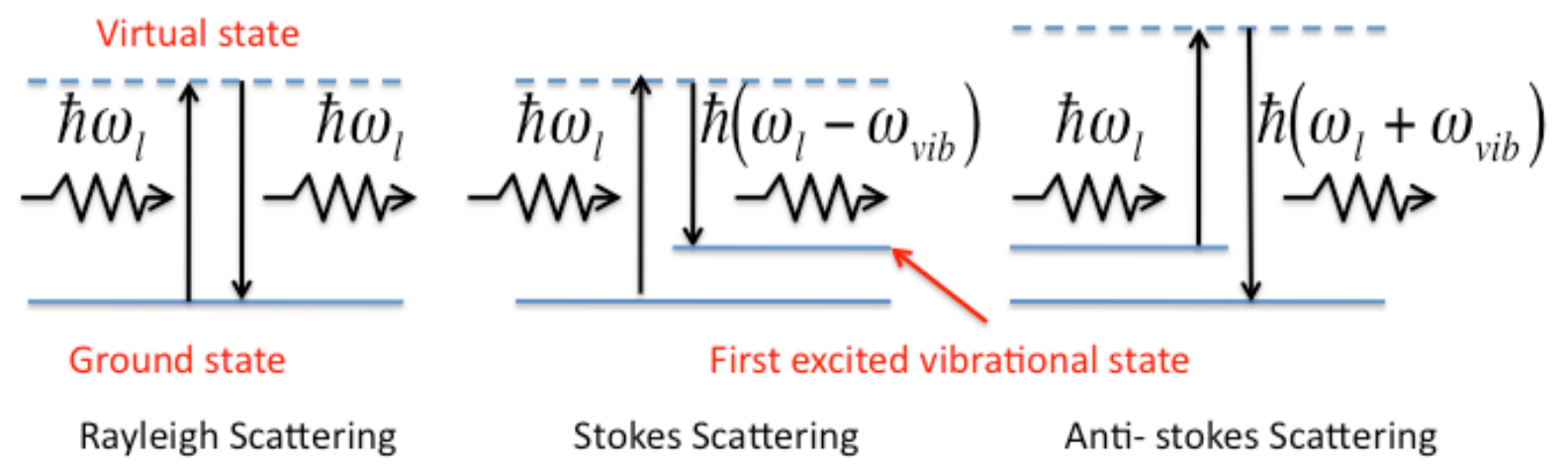

Figure 3.1. Diagram of Rayleigh and Raman scattering processes. Here $\omega_{1}$ is the angular frequency of the incident radiation and $\omega_{\text {vib }}$ is the angular frequency of the molecular vibration.

It is important to note that the Raman effect consists the interaction of an incident photon with a change in polarizability of the molecule. Of course, light may also interact with molecular vibrations via the absorption by resonant dipole oscillations, as is used in infrared spectroscopy. However, while Raman spectroscopy similarly probes vibrational modes, individual vibrational modes are often either Raman or infrared active. To show this ${ }^{18}$, we start by noting that the dipole moment of an irradiated molecule is expressed as

$$
P=\alpha E
$$

where $\alpha$ is the polarizability. The electric field of the incident radiation can be taken to be

$$
E=E_{0} \cos \left(\omega_{l} t\right)
$$

$\alpha$ is a function of each normal vibrational mode as given by

$$
\alpha=\alpha_{0}+\left(\frac{\partial \alpha}{\partial q}\right)_{0} q,
$$


where $\alpha_{0}$ is the permanent polarizability and $\mathrm{q}$ is the normal coordinate. For simplicity, we only keep the first tow terms of the Taylor expansion here. For small vibrations, q can be taken to oscillate harmonically as

$$
q=q_{0} \cos \left(\omega_{v i b} t\right)
$$

Combining equations $3.1-3.4$, one reaches the following expression for the dipole moment of a molecule with its $n^{\text {th }}$ normal mode excited:

$$
P=\mu_{0}+\alpha_{0} E_{0} \cos \left(\omega_{l} t\right)+\left(\frac{\partial \alpha}{\partial q}\right)_{0} E_{0} q_{0} \cos \left(\omega_{l} t\right) \cos \left(\omega_{v i b} t\right)
$$

Here, the second term corresponds to Rayleigh scattering and the third term corresponds to Raman scattering. The last term can be rewritten as

$$
\frac{1}{2}\left(\frac{\partial \alpha}{\partial q}\right)_{0} E_{0} q_{0} \cos \left(\left(\omega_{l}-\omega_{v i b}\right) t\right) \cos \left(\left(\omega_{l}+\omega_{v i b}\right) t\right)
$$

using the common trigonometric identity. This clearly shows how the molecule will reradiate at energies shifted by the vibrational energy. It should be noted that the Raman spectrum may be obtained via an excitation from an arbitrary wavelength (unlike the IR spectrum). However, typically a laser in the near UV to near infrared range is used. In these cases, the energy of the incident photon is much, much greater than the energy of the vibration. Thus, the Raman shift will result in a small change in the energy of the scattered photons.

One should pay special attention to the criteria for determining if the $\mathrm{n}^{\text {th }}$ normal mode will be Raman or IR active. From equation 3.5, it is clearly seen that in order for a vibration to be Raman active, the term $\left(\frac{\partial \alpha}{\partial q}\right)_{0}$ must be non-zero. That is to say the polarizability of the molecule must change as a function of the vibration in such a way that the polarizability derivative is non-zero at the center point. On the other hand, a 
vibration will be IR active only if there is a change in the dipole moment of the molecule as a function of the vibration. For molecules with a symmetry center, these two distinct criteria result in selection rules that require a vibration be either Raman active or IR active. Particularly, symmetric vibrations will be IR active and anti-symmetric vibrations will be Raman active. These selection rues are demonstrated in Figure 3.2 where two vibrational modes of the $\mathrm{CO}_{2}$ molecule are shown. Here, the symmetric vibration does not change the dipole moment of the molecule (it stays zero throughout), but does change the polarizability. On the other hand, the second vibration does feature a changing dipole moment. While the polarizability of the molecules does change during the second vibration, it changes identically on both sides of the minimum, giving a polarizability derivative of zero. Clearly, it is seen that disrupting or changing the symmetry of molecules can have drastic results on the Raman spectrum by means of making particular vibrational modes either Raman active or silent. 


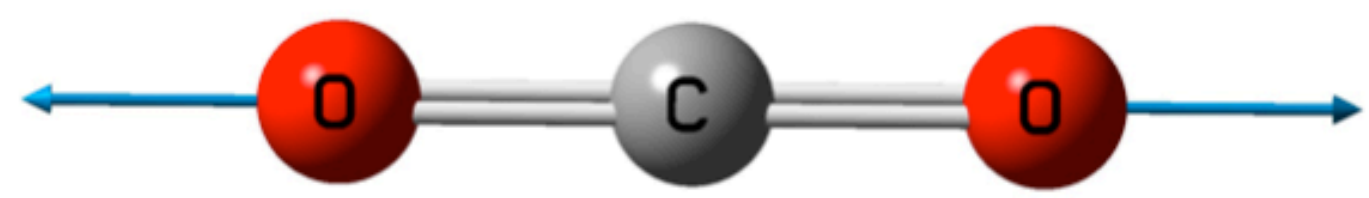

(a)

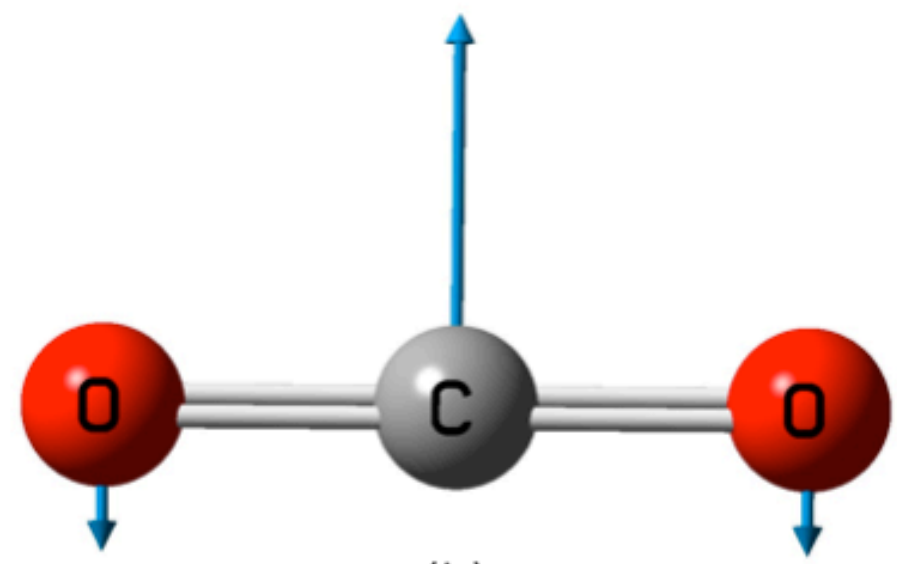

(b)

Figure 3.2. Two vibrations of the carbon dioxide moelecule. (a) a symmetric vibration where the dipole moment of the molecules does not change but the polarizability anisotropically. This mode is only Raman active. (b) a vibration where the dipole moment changes but the polarizability does not change anisotropically. This mode is only infrared active.

Since the Raman effect is dependent on the polarizability derivative, it follows that the intensities of the Raman peaks are theoretically obtained from the first derivatives of the polarizability with respect to the nuclear coordinates. The Raman scattering cross section is given by ${ }^{19}$ : 


$$
\frac{d \sigma}{d \Omega}=\frac{\omega_{l} \omega_{s}^{3}}{c^{4}} \frac{\hbar}{2 \omega_{f}}\left[\left\langle n\left(\omega_{f}\right)\right\rangle+1\right]\left|P_{f}\right|^{2} L
$$

where $<n\left(\omega_{f}\right)>$ is the mean occupation number:

$$
\left\langle n\left(\omega_{f}\right)\right\rangle=\left[\exp \left(\beta \hbar \omega_{f}\right)-1\right]^{-1}
$$

here: $\omega_{f}$ is the Raman shift frequency (in $\mathrm{rad} / \mathrm{s}$ ), $\omega_{l}$ is the incident excitation frequency (in $\mathrm{rad} / \mathrm{s}$ ), $\omega_{s}$ is the scattered frequency (in $\mathrm{rad} / \mathrm{s}$ ), $\left|P_{\mathrm{f}}\right|^{2}$ is the Raman activity (in $\mathrm{cm}^{4} / \mathrm{g}$ ), and $\mathrm{L}$ is the local field correction. The Raman activity (differential scattering cross section) is usually written in terms of the isotropic and the anisotropic polarizability derivatives, which is given by $\frac{d \sigma_{\text {total }}}{d \Omega}=\frac{\omega^{4}}{c^{4}}\left[\alpha^{2}+\frac{7}{45} \gamma^{2}\right] ; \alpha$ being the mean polarizability derivative and $\gamma$ is the anisotropic polarizability derivative.

\subsection{Surface Enhanced Raman Scattering}

Surface enhanced Raman spectroscopy (SERS) is a specific type of Raman spectroscopy in which metallic nanoparticles are used to enhance the Raman signal by many orders of magnitude via the effect of their surface plasmons. The electromagnetic field enhancement due to the local field of surface plasmons excited by incident light is a major contribution to the SERS effect. ${ }^{20,21}$. For example, gold nanoparticles emit in the near-infrared portion due to their surface plasmons. Other coinage metals such as copper and silver emit at higher energies. ${ }^{22}$ If near-infrared light is incident on a sample containing gold nanoparticles, both the local incident and scattered fields (since $\omega_{\mathrm{i}} \approx \omega_{\mathrm{s}}$ ) at the point of scattering will be greatly enhanced resulting in a much larger Raman signal. A direct chemical interaction via a charge transfer complex can also lead to the

SERS effect. ${ }^{23}$ The total SERS effect, including enhancement by both local fields and 
charge transfer complexes, is pictorially shown in Figure 3.3. Here, the incident and scattered fields are shown by perpendicular arrows. $A\left(v_{L}\right)$ and $A\left(v_{S}\right)$ represent the field enhancement factors due to the excitation of the surface plasmons on the metallic nanoparticles. The enhancement due to the chemical coupling of the metallic nanoparticles with the analyte, on the other hand, is expressed as an increased Raman scattering cross section $\sigma_{a d s}^{R}$. One can see how the effects, one electromagnetic and one chemical, can independently contribute to the SERS effect.

While SERS spectra may clearly be obtained by dispersing metal nanoparticles through the analyte, a similar effect may be obtained by using nanostructured metal surfaces as well. As long as the structure, be it ridges, wells, or protrusions, is on the order of tens of nanometers, it will support surface plasmons capable of producing the SERS effect. Thus, one can imagine how the interface between a rough metal film and a soft organic film may lead to an SERS enhancement of the Raman spectrum of the organic film. 

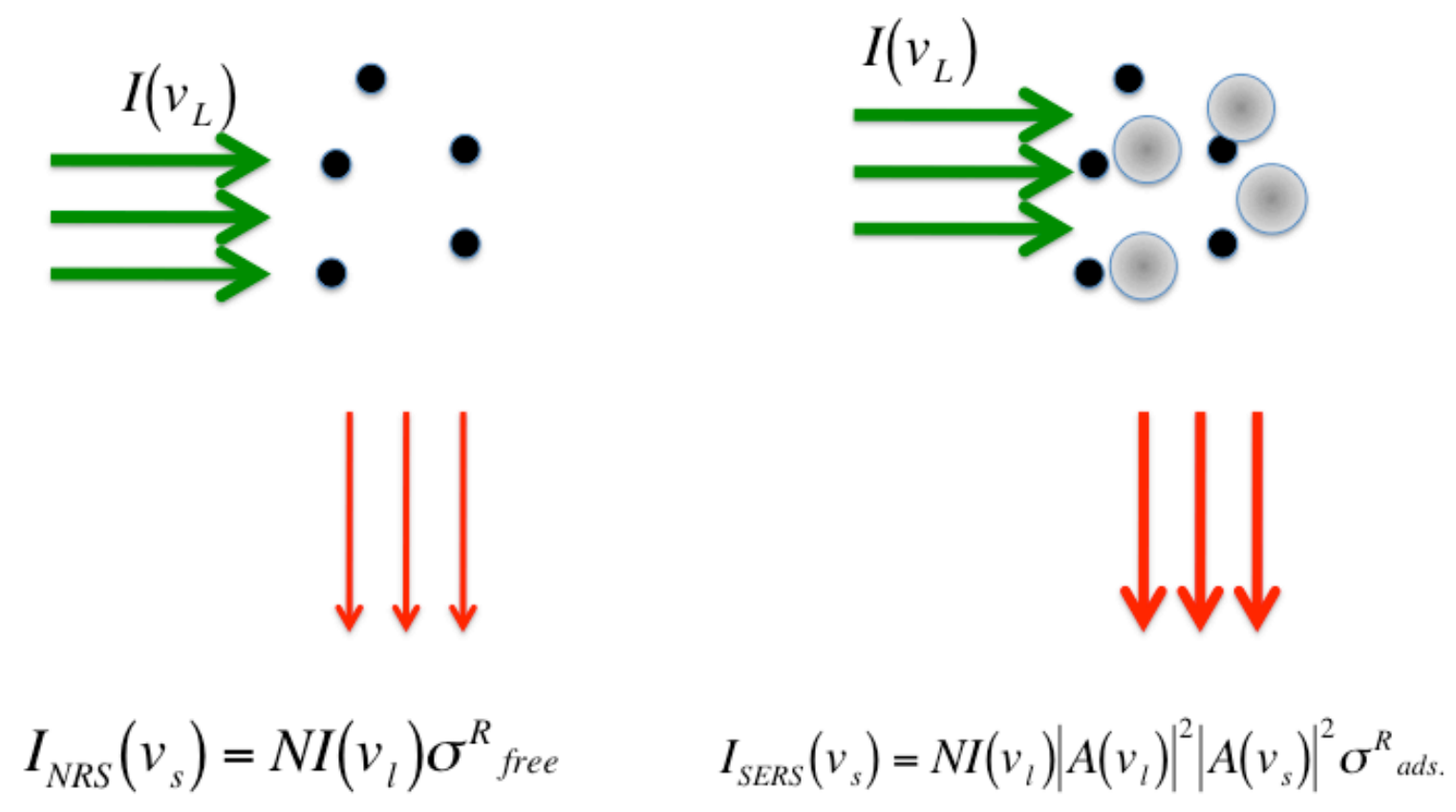

Figure 3.3. The SERS effect. Here $I\left(v_{L}\right)$ and $I_{S E R S}\left(v_{L}\right)$ are the incident and scattered intensities, respectively. $\sigma^{R}$ free and $\sigma^{R}$ ads. are interaction cross sections of the free and the adsorbed (via a charge transfer complex) molecules, respectively. $A\left(v_{L}\right)$ and $A\left(v_{S}\right)$ are the field enhancement factors are the incident and scattered frequencies, respectively.

\subsection{Density Functional Theory}

Density functional theory (DFT) is a powerful theoretical method for electronic structure calculation in solid state physics and chemistry. It allows for the calculation of electronic structure at much lower computational cost than many other methods. DFT achieves this by mapping the full, interacting problem to a non-interacting problem that is easier to solve. In addition to the electronic structure, DFT allows one to theoretically predict properties, such as polarizabilities and vibrational frequencies, of any arbitrary molecule. 
The difficulty in solving the Schrodinger equation for the interacting system arises from the electron-electron interaction. In fact, as any first year graduate student in physics will testify to, solving for the electronic structure of even the simplest (save hydrogen) atoms becomes tedious and time consuming. One can imagine how the computational requirements of such calculations grow as systems become more complex. However, theoretically one can use numerical methods to solve any such problem and obtain a wavefunction (and thus a particle density) for the ground state of the system. DFT is based on the realization that from this density one can extract all vital parameters of the system and, that if one had an alternate means of obtaining this density, one could perhaps obtain these parameters without the computational pain required in solving the Schrodinger equation. In 1965, Kohn and Hohenberg stated the following theorem: (1) the ground state density uniquely determines the potential and all properties of the system. (2) there exists a universal functional of density (independent of external potential) that gives the minimal value of energy, which is the ground state energy. ${ }^{24}$ Thus, the groundwork was laid for a method that yielded an alternate path to calculating a system's ground state density.

Rather than solving for the exact wavefunction, DFT solves for what is known as the Kohn-Sham orbitals. ${ }^{25}$ The Kohn-Sham wavefunction is determined by imagining that there are non-interacting electrons whose density is exactly identical to the electron density of the real physical system. The calculation begins by providing a simple guess for the ground state density in the form of a basis set. Simple approximations of the dependence of energy on the density, known as functionals, can then be applied to determine the potential required to create this non-interacting electron density. Solving 
the Kohn-Sham Hamiltonian for this potential provides a refined density. Once this iterative approach has converged to a final density, the problem has been solved. Since the computed (non-interacting) electron density is identical to the true (interacting) electron density, the relatively simple calculation can return electronic structure information that would otherwise be computationally prohibitive. One must note that since the problem is being solved with non-interacting electrons, the energy functionals must include an exchange correlation term with corrects for the exchange correlation energy.

The Kohn-Sham energy functional takes the following form:

$$
E[\rho]=T_{s}[\rho]+\int v_{e x t}(\vec{r}) \rho(\vec{r}) d \vec{r}+V_{C}[\rho]+E_{X C}[\rho]
$$

where $T_{s}[\rho]$ is the kinetic energy, $v_{\text {ext }}$ is the external potential (from the nuclei, for example), $\mathrm{V}_{\mathrm{C}}$ is the Coulomb energy term, and $\mathrm{E}_{\mathrm{XC}}$ is the exchange-correlation energy. The exchange energy is essentially a manifestation of the Pauli exclusion principle. That is to say, when when two wavefuncitons of like spin overlap, the spin repulsion must be accounted for in addition to the Columbic repulsion. The correlation energy, on the other hand, is defined as the remaining unknown portion of the energy. ${ }^{26}$ The exchangecorrelation energy is usually formed using either the localized density approximation (LDA) or the generalized gradient approximation (GGA), where the exchange-correlation energy is assumed to depend exclusively on the local electronic density or both the local electronic density and the gradient of the density, respectively.

We use the Gaussian software package ${ }^{27}$ for all of our DFT calculations. We use the $3-21 \mathrm{G}^{*}$ basis set and the B3LYP (Becke's three parameter hybrid) ${ }^{28}$ functionals to perform geometry optimization and calculations of force constants, dipole moments, and 
polarizability derivatives. $3-21 \mathrm{G}^{*}$ is a split double zeta polarized basis set. ${ }^{29}$ That is, each core orbital is comprised of 3 Gaussian functions and each valence orbital is comprised of two functions, consisting of two and one Gaussian functions, respectively. B3LYP is a hybrid functional which includes the exchange correlation correction. The B3LYP functional takes the following form ${ }^{28}$ :

$$
E_{X C}^{B 3 L Y P}=E_{X C}^{L D A}+a_{0}\left(E_{X}^{H F}-E_{X}^{L D A}\right)+a_{x}\left(E_{X}^{G G A}-E_{X}^{L D A}\right)+a_{c}\left(E_{C}^{G G A}-E_{C}^{L D A}\right)
$$

where $\mathrm{a}_{0}=.20, \mathrm{a}_{\mathrm{x}}=.72$, and $\mathrm{a}_{\mathrm{c}}=.81$. Here $\mathrm{E}^{\mathrm{HF}}$ refers to the Hartree-Fock exact exchange functional.

While the $3-21 \mathrm{G}^{*}$ basis set provides an ideal combination of speed and accuracy for computing the electronic structure of the organic semiconductor molecules discussed in this work, it is not usable for transition metals. For those cases where the electronic structure of a metal-semiconductor complex was needed, the LANL2DZ (Los Alamos National Laboratory 2 Double-Zeta) basis set was used. The LANL2DZ is an effective core potential (ECP) basis set, used for atoms heavier than Ne. ${ }^{30}$ In an ECP basis set, the core electrons are replaced by an effective core potential, which incorporates relativistic effects. This allows for valence electron calculations to be carried out in a fraction of the computational time that would otherwise be required. ${ }^{31}$ We used the LANL2DZ basis set for $\mathrm{Au}$ atoms and the $3-21 \mathrm{G}^{*}$ basis set for $\mathrm{C}$ and $\mathrm{H}$ atoms. Using a combination of $\mathrm{ECP}$ basis set for transition metal elements and an all electron basis set for all other elements is increasingly popular. ${ }^{32}$

The Raman spectrum (frequencies and intensities) calculations were performed in two steps; first the force constant matrix was evaluated and then the eigenvalue equation was solved to obtain the eigenvalues and eigenvectors. The Gaussian output is in terms of 
the Raman activity, which is essentially the square of the polarizability tensor. In order to compare the theoretical spectrum with experiment, one needs to compute the Raman scattering cross section. This can be done using equation 3.7. It should be noted that Gaussian returns Raman activity in $\AA^{4} / \mathrm{amu}$ and Raman shift in wavenumbers. Furthermore, the excitation source is usually given in nanometers. It is important that the above values must all be converted to the correct units before inserting them into the equation for the Raman cross section. 


\section{Experimental Techniques}

\subsection{Fabrication of OFETs}

\subsubsection{Substrate preparation}

Device performance in OFETs is dominated by two interfaces: the semiconductordielectric interface and the metal-semiconductor interface. While the latter interface plays an important role in charge injection, the quality of the semiconductor-dielectric interface is absolutely critical to device performance. In bottom gate OFETs, where the semiconductor is deposited immediately on top of a previously formed dielectric layer, the nature of the dielectric surface is crucial in determining how well ordered the semiconductor film will be and what density of charge carrier traps there will be. In fact, it has been said that the choice of the gate dielectric is as important to device performance as the choice of the semiconductor itself. ${ }^{33}$ Much work has been done in using surface treatments to improve the dielectric surface prior to semiconductor deposition. This section contains a review of some of this work, followed by the substrate preparation recipe that was developed for all subsequent OFET fabrication.

Pentacene OFETs fabricated using a $\mathrm{SiO}_{2}$ dielectric have been shown to be limited in performance due to trap states at the pentacene/ $\mathrm{SiO}_{2}$ interface. ${ }^{34}$ Specifically, while pentacene hole mobilities were found to approach that of amorphous Silicon, the organic devices suffer from high threshold voltages (in the tens of volts). Cleaning the substrates via oxygen plasma helps reduce but not eliminate the effects of these traps. Since thorough cleaning cannot eliminate these effects, one expects that the trap states are an inherent limitation of the hydrophilic $\mathrm{SiO}_{2}$ dielectric. It is worth noting that in all these 
systems, the semiconducting materials preform better when deposited on hydrophobic surfaces. This implies that the origin of the trap states is related to the hydrophilicity of the $\mathrm{SiO}_{2}$ surface.

Since the $\mathrm{SiO}_{2}$ surface is hydrophilic because of surface silanol ( $\left.\mathrm{Si}-\mathrm{O}-\mathrm{H}\right)$ groups, one may ask if these groups are responsible for trapping mobile charge carriers. Indeed, it has been shown that hole mobility is inversely related to silanol density in OFETs fabricated on $\mathrm{SiO}_{2}$ dielectrics. ${ }^{35}$ Additionally, hydroxyl groups in organic dielectrics were found to have a similar, if weaker effect. Furthermore, silanol groups trap electrons even more strongly, completely inhibiting n-type transport for many semiconductors. ${ }^{36}$ Clearly, the adverse effect of the surface silanol groups must be lessened in order to fabricate optimal $\mathrm{SiO}_{2}$ based devices. Additionally, since organic semiconductors, being based on conjugated hydrocarbon backbones, are mainly hydrophobic, it is reasonable to expect the quality of the semiconductor-dielectric interface is optimized when they are grown on hydrophobic, rather than hydrophilic dielectric surfaces.

Converting the hydrophilic $\mathrm{SiO}_{2}$ surface to a hydrophobic one can be done by covering the surface with a thin, hydrophobic surface treatment. A suitable treatment should consist of a molecule that has a hydrophilic head that can be anchored to the $\mathrm{SiO}_{2}$ surface and a hydrophobic tail that will provide a new surface for the organic semiconductor to be deposited on. Ideally, the surface treatment should form as a selfassembled monolayer (SAM) on the $\mathrm{SiO}_{2}$ as well, thus ensuring it is uniform and thin. A commonly used surface treatment that meets these requirements is Octadecyltrichlorosilane (OTS). The molecular structure of OTS is shown in Figure 4.1. Here, the trichlorosilane head of the molecule serves as the hydrophilic anchor and the 
18-carbon aliphatic chain serves as the hydrophobic tail. The OTS surface treatment has been shown to decrease the surface energy of the dielectric (as one would expect for a hydrophobic treatment), resulting in greater crystallinity of subsequently deposited organic semiconductor films. ${ }^{37}$ The more order films then allow for increased charge carrier mobilities.

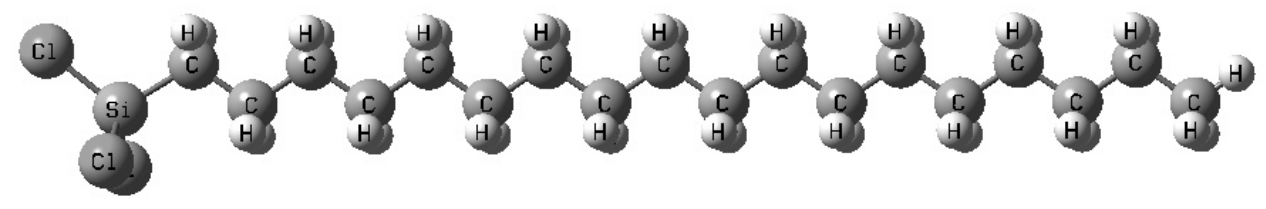

Figure 4.1. Chemical Structure of OTS

The OTS SAM is most commonly formed by dipping the substrates in an OTS solution. The OTS SAM is formed via the following two steps ${ }^{38,39}$ : (1) small amounts of ambient water lead to the hydrolysis of the chloro- moieties of the silane, resulting in trisilanol species; (2) the trisilanol species polymerize onto the dielectric surface. A diagram of the final SAM is shown in Figure 4.2. Note how the crosslinking of both OTS molecule to OTS molecule and OTS molecule to the $\mathrm{SiO}_{2}$ substrate ensures a monolayer is formed. Since the SAM formation occurs via water induced hydrolysis and polymerization steps, it is logical to expect that water concentration plays a vital role in SAM formation. Indeed, it is found that a high quality OTS SAM is only formed on hydrated $\mathrm{SiO}_{2}$ surfaces ${ }^{40}$, where excess water extracted from the surface into the solvent is free to participate in the hydrolysis of OTS.$^{41}$ Catalytic water may also be introduced into the system via dissolved water in the solvent. SAM coverage is found to be a 
function of solvent water concentration, with water-saturated solvent giving the best results. $^{42}$

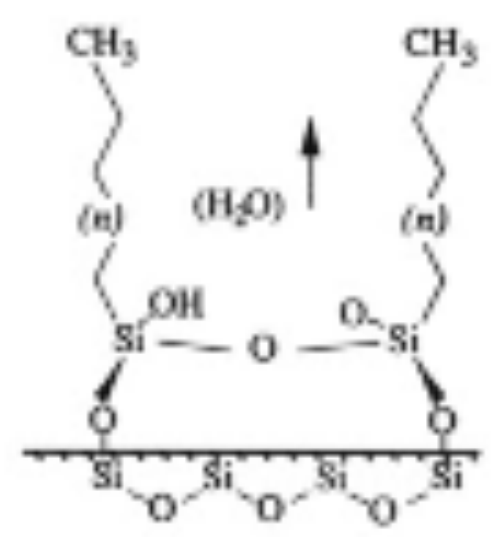

Figure 4.2 A diagram of two OTS molecules forming a portion of the OTS SAM on $\mathrm{SiO}_{2}$. Note how a water molecule is released as the OTS molecules polymerize. ${ }^{43}$

Clearly, since the assembly of the OTS monolayer proceeds via a water catalyzed pathway, careful consideration must be paid to variables such as hydration level of the substrate, water concentration in the solvent, age of the OTS solution, and deposition time when designing a recipe for OTS SAM deposition. The protocol for substrate cleaning and OTS deposition presented in the next section was developed by carefully varying these parameters until optimal OTS coverage was obtained. Even slight alterations to the protocol can have drastic effects on the quality of the resultant substrates.

$\mathrm{Si}^{++} / \mathrm{SiO}_{2}$ substrates were first cleaned via an organic cleaning in order to remove soluble organic impurities. Typically, no more than 4 pieces were prepared at one time. 
Immediately after being cleaved into 1 in. $\mathrm{x} 1 \mathrm{in}$. pieces, the substrates were quickly rinsed in isoproyl alcohol to wash away any loose Silicon dust left by the diamond scribe. Substrates were then immediately submerged in $\sim 50 \mathrm{ml}$ of acetone and ultrasonicated for 20 minutes. Exposure to ultrasonic $(>20 \mathrm{kHz})$ waves causes small bubbles in the acetone to form and collapse on the substrate surface, thereby knocking loose and dissolving organic contaminants. After ultrasonication, the substrates were removed from the acetone bath and quickly rinsed with copious amounts of acetone followed by isopropyl alcohol. All traces of the organic solvents were then removed from the substrates by rinsing with deionized water, in preparation for the subsequent acid cleaning step.

While the previous organic cleaning step effectively removes soluble organic impurities, it cannot remove ionic contaminants. Furthermore, heavier organic residues may be insoluble in acetone and isopropyl alcohol, even during ultrasonication. To clean the substrates of these impurities, a mixture of $7: 3$ sulfuric acid $\left(\mathrm{H}_{2} \mathrm{SO}_{4}\right)$ to hydrogen peroxide $\left(\mathrm{H}_{2} \mathrm{O}_{2}\right)$ was used. This solution, commonly known as Piranha, is a highly acidic, strongly oxidizing agent used to clean organic and ionic impurities off substrates. When sulfuric acid and hydrogen peroxide are mixed, the acid forces the dissociation of the peroxide via protonation:

$$
\mathrm{H}_{2} \mathrm{SO}_{4}+\mathrm{H}_{2} \mathrm{O}_{2} \rightarrow \mathrm{HSO}_{4}^{-}+\mathrm{H}_{3} \mathrm{O}^{+}+\mathrm{O}
$$

The resultant atomic oxygen is a highly reactive species capable of aggressively oxidizing carbon-bearing residues. The piranhna, in effect, aggressively eats away organic residues from the surface of the substrates. It should be noted that this reaction is very exothermic. Thus the piranha solution is a highly acidic oxidizing agent capable of heating itself to temperatures well above the boiling point of water. It must be treated 
with extreme care. It should go without saying that the piranha is absolutely incompatible with all flammable organic solvents and will react explosively with them. Thus, for all subsequent cleaning steps, all organic solvent bottles were removed from the work area to prevent accidental mixing.

Immediately after the organic cleaning and water rinse, the substrates were submerged in a heated piranha solution for 5 minutes. The piranha solution was made by adding $9 \mathrm{ml}$ of $\mathrm{H}_{2} \mathrm{O}_{2}$ to $21 \mathrm{ml}$ of $\mathrm{H}_{2} \mathrm{SO}_{4}$. To ensure the solution was ready when needed, the solution was prepared during the organic cleaning step. The prepared solution was then heated on a hot plate to $125^{\circ} \mathrm{C}$. The elevated temperature drives the reaction given in eq. 4.1 to the right, ensuring an adequate amount of the reactive oxygen species is present in the solution. After five minutes in the heated solution, the substrates were removed using plastic forceps and thoroughly washed with deionized water. Compressed air was then used to dry the substrates. The highly hydrophilic nature of the substrate surface, which was a direct result of the strongly protonating piranha solution, was evident in the way water sheeted across the substrate surface. The substrates were placed in boxes and moved to a glovebox for storage.

As discussed previously, the hydrophilic, silanol-bearing $\mathrm{SiO}_{2}$ is far from ideal for growing organic semiconductor films. Therefore, an OTS SAM was formed on the substrates as per the following protocol. The OTS SAM was deposited 24 hours after the cleaning procedure. As the substrates emerge from the cleaning procedure in a very hydrated state, it is vital to wait one day before beginning the OTS surface modification step. If OTS SAM deposition is attempted immediately after cleaning, the excess water on the $\mathrm{SiO}_{2}$ surface will cause a runaway polymerization reaction in the OTS solution 
resulting in poor monolayer formation. Attempts were made to adjust the OTS recipe to allow for immediate deposition, but it was found waiting 24 hours gave more reliable results.

The OTS solution was prepared in freshly cleaned and dried glassware. Glassware was cleaned using Liquinox Critical Cleaning Detergent (Alconox Inc.) and dried at $120^{\circ} \mathrm{C}$. The glassware was then allowed to come back to room temperature before use. OTS ( $>90 \%$, Sigma Aldrich) was dissolved in HPLC grade toluene ( $>99.9 \%$, Sigma Aldrich) at a concentration of $.25 \mathrm{~g} / 10 \mathrm{ml}$. Typically, $\sim .75 \mathrm{~g}$ of OTS and $\sim 30 \mathrm{ml}$ of toluene was used in a small petri dish. The solution was allowed to age 2-3 minutes, and then each substrate was submerged in the solution for 30 seconds. The substrate was removed and rinsed with copious amounts of HPLC grade toluene. The substrate was then submerged in fresh HPLC grade toluene while the remaining substrates were treated in the OTS solution. It should be noted that the insertion of each substrate degrades the OTS solution to the point that it can be only be used for 3-4 substrates. After all the substrates had been treated, they were ultrasonicated in toluene for 30 seconds. Finally the completed substrates were rinsed with isopropyl alcohol and dried with compressed air.

Upon completion of the OTS procedure, the $\mathrm{SiO}_{2}$ surfaces should be clean without any cloudy residue. Such a residue indicates the substrate was exposed to a runaway OTS polymerization reaction, likely caused by the introduction of excess water into the reaction solution. A substrate thus exposed may not be usable for device fabrication. Furthermore, a successful OTS monolayer formation may be confirmed by placing a drop of water on the surface and examining the contact angle the drop forms with the surface. It has been shown that the contact angle should be $\sim 80^{\circ} .{ }^{37} \mathrm{~A}$ contact angle less than this is 
indicative of incomplete OTS coverage. If the above described protocol ever results in incompletely covered substrates during a particular a session, it may be modified by dipping the substrates for a longer time (up to 60 seconds).

The complete cleaning and OTS surface modification procedure is outlined in Figure 4.3. Upon completion, the substrates were ready for device fabrication.

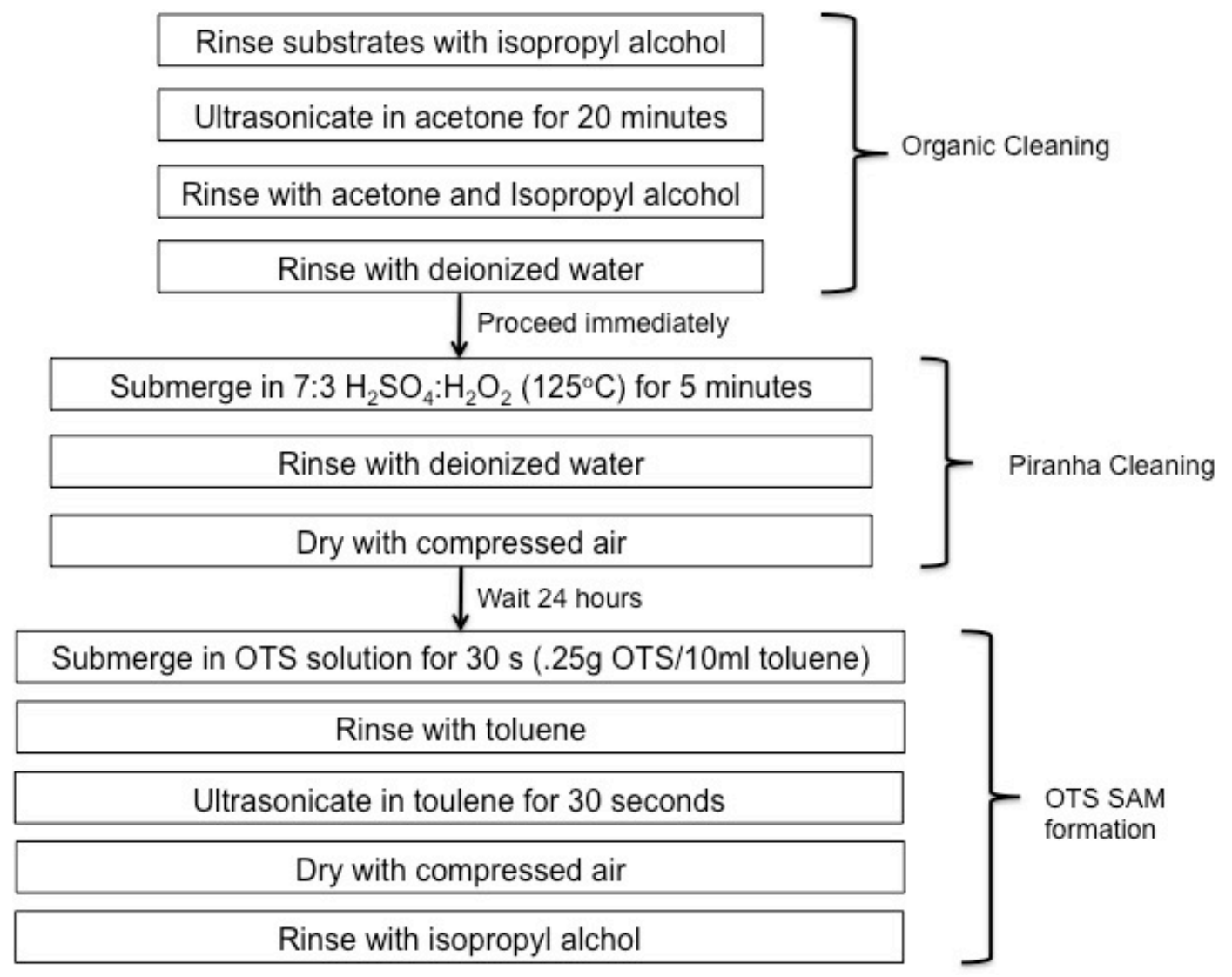

Figure 4.3. Flow chart for $\mathrm{Si}^{++} / \mathrm{SiO}_{2}$ substrate cleaning and surface modification procedure. 


\subsubsection{Film growth and Contact deposition}

Once the $\mathrm{SiO}_{2} / \mathrm{Si}^{++}$substrates have been prepared, the organic semiconductor must be deposited so as to form a thin film on top of the $\mathrm{SiO}_{2}$ dielectric. There are various methods of forming a suitable film. At the very least, the chosen method should give a uniform, well ordered film where the constituent semiconductor molecules are left undamaged. Ideally, the method should also be quick and easily customizable such that it can be applied to many different semiconductor and dielectric systems. Three such methods are described in this chapter: spin-coating; thermal evaporation; and matrixassisted pulsed laser deposition (MAPLE). Generally speaking, each of these methods has its restrictions on which materials it may be used with. For example, it may be possible to deposit a film of a certain materials using thermal evaporation, while it is not possible to spin-cast a film of that material.

After the semiconductor film has been formed, metal contacts must be deposited on top of the film. All devices discussed in this work have their top metal contact deposited via thermal evaporation. Thus, the section describing thermal evaporation is applicable to both semiconductor materials and metals.

In this work, an MBraun glovebox containing a thermal evaporation system was used for most device fabrication. The glovebox contains an inert atmosphere of dry nitrogen with concentrations of $\mathrm{O}_{2}$ and $\mathrm{H}_{2} \mathrm{O}$ held to $\sim 2$ ppm. Figure 4.4 shows a schematic of the glovebox. Briefly, the glovebox system consists of two boxes: box A contains a Direct 1000 spin-coater, an oven capable of $>300^{\circ} \mathrm{C}$, and an activated carbon-based solvent vapor removal column; box B contains the thermal evaporation system. The two boxes are connected via a common gas circulation and purification system. Three antechambers 
are used to insert and remove samples from the two boxes as well as transfer them from one box to the other. The purification system consists of a copper catalyst bed. The copper is able to sequester oxygen and sulfur as $\mathrm{CuO}_{\mathrm{x}}$ and $\mathrm{CuSO}_{4}$, respectively, thus maintaining the inert atmosphere. The copper bed must periodically be regenerated by passing hydrogen gas over it, extracting the sequestered impurities as $\mathrm{H}_{2} \mathrm{O}$ and $\mathrm{H}_{2} \mathrm{~S}$.

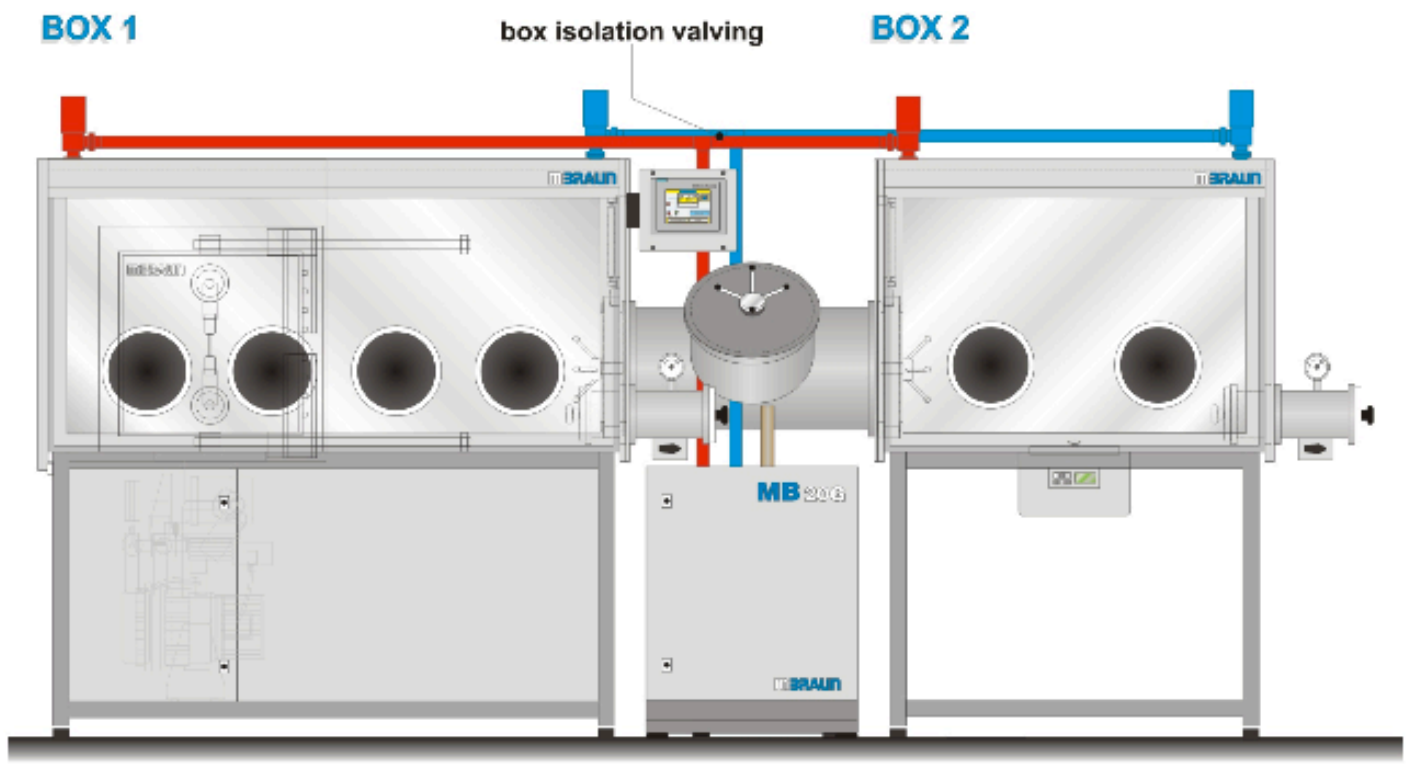

Figure 4.4. Schematic of the MBraun glovebox system.

\subsubsection{Spin-Coating}

Spin-coating is a typical thin-film deposition method for polymeric, soluble semiconductors and dielectrics. Spin-coating allows for the quick formation of uniform films by taking advantage of the solubility of such materials. Therefore, this method is restricted to those organic semiconductors and dielectrics that are appreciably soluble in a solvent. While many semiconducting polymers can be made soluble by the addition of solubizing side chains, small molecule organic semiconductors are completely insoluble in common solvent and thus cannot be spun-cast. It also may only be used in cases where 
the chosen solvent does not interact with or degrade the substrate on which the film is being formed. Conveniently, the $\mathrm{SiO}_{2}$ surface is completely inert to and unaffected by the organic solvents commonly used to dissolve organic semiconductors.

The spin-coating process may be summarized as follows: (1) the organic semiconductor is dissolved in a solvent to a chosen concentration; (2) a predetermined volume of the solution is dripped onto the selected substrate (which may be heated) either as it begins to rotate, or before it has began rotating; (3) the substrate rotates at a speed of a few thousand revolutions per minute (rpm); (4) the solution washes out over the surface of the substrate, leaving behind a film as it evaporates. Typically, the substrate, along with the newly formed film, will then be baked at an elevated temperature (often under vacuum) to drive off any remaining solvent left in the film. The spin-coating process, as described above, leaves ample opportunity for customization for different polymeric materials, solvents, and substrates. In fact, small changes in any of the steps may have drastic implications on film thickness, coverage, or uniformity. For example, the film may be made thicker by using a more concentrated solution, using a larger volume of solution, using a lower boiling point solvent, or by spinning at a lower speed. For some polymeric materials, there may be restrictions on usable solvents and maximum obtainable concentration (via the materials solubility). Furthermore, on some highly hydrophobic substrates, there may be a limitation to the lowest usable spin-speed, as at low speeds the solution may simply form a bead and be flung off the substrate without leaving a film. Clearly, an individual recipe must be developed for each semiconductor/dielectric and substrate system by carefully optimizing each of the above 
described steps. This is, in general, a non-trivial process. Details on the spin-coating conditions for the systems discussed in the work are found in their respective chapters.

Figure 4.5 shows optical images representative of common outcomes when spincoating films. Figure 4.5 (a) shows a properly formed film. One sees a uniform, evenly colored film covering the vast majority of the substrate. Figure 4.5 (b), on the other hand, shows the result of a failed spin-coating attempt. The poor coverage of and radial features in the film are indicative of very poor wetting of the semiconductor solution on the hydrophobic substrate. The spin speed in this case was not sufficient for spin-coating on such a substrate. The polymer used to make the films seen in Figure 4.5 (a) and (b) is a polyflourenes based compound, as discussed in chapter 5. Figure 4.5 (c) shows a magnified image of a heavily nucleated semiconductor film. The polymer used to make this film was only marginally soluble in the chosen solvent, resulting in pre-mature precipitation of the solute. Further filtering of the solution prior to spin-coating, may have helped in this case. This film is not suitable for device fabrication. The material used to make this film is also a polyflourenes based compound. 


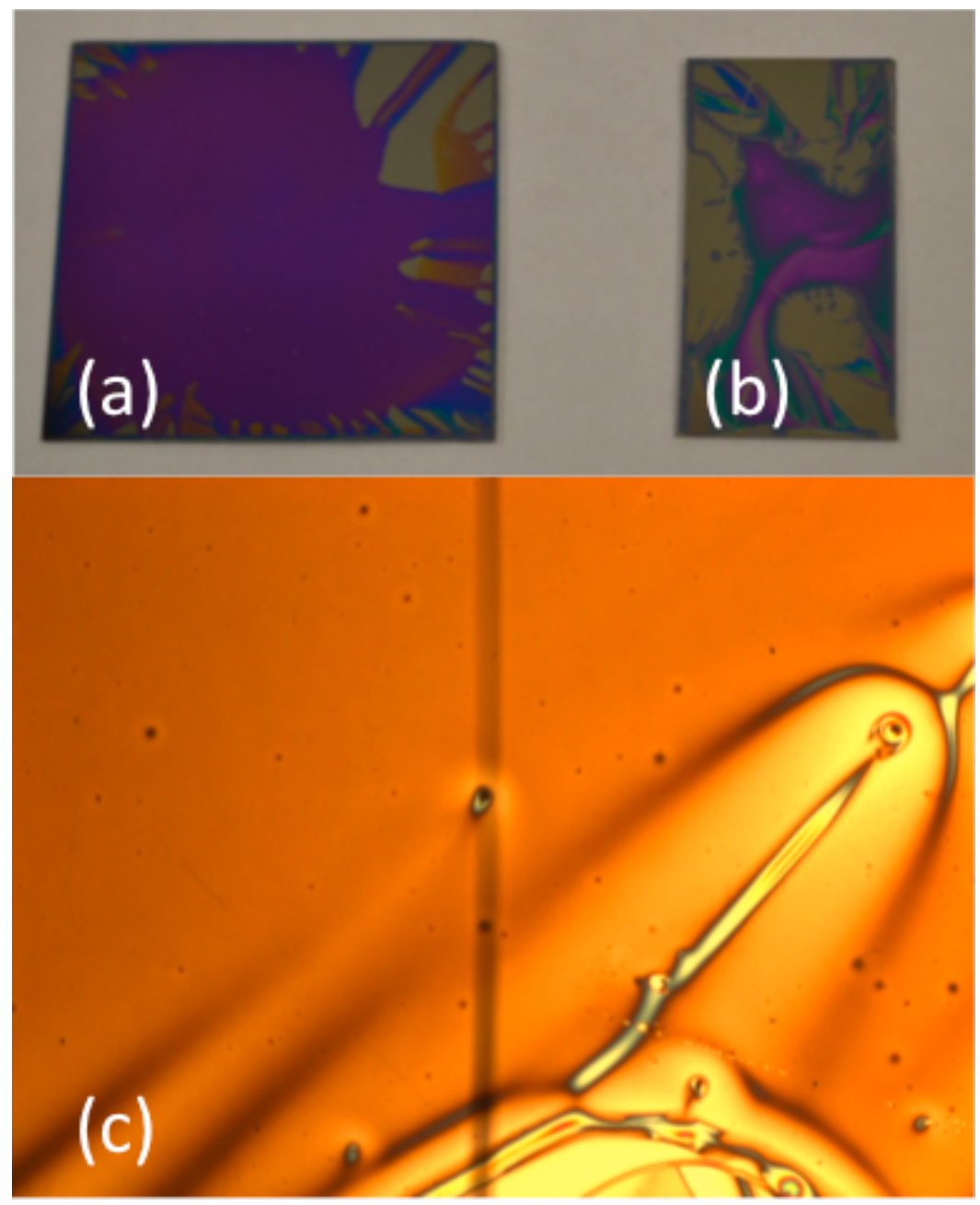

Figure 4.5. Images of spun-cast semiconductor films: (a) a well formed film covering most of the substate; (b) a poorly formed film unsuitable for further use; (c) a magnified image of a heavily nucleated film.

\subsubsection{Thermal evaporation}

Thermal evaporation is a form of physical vapor deposition where a solid source is heated to the point where it vaporizes, creating a vapor which then condenses on the target substrate. Clearly, this process is only usable for those materials that have a 
melting or sublimation temperate much less than their decomposition temperature. Thus, the process is suitable for many metals and small organic molecules, while it is not usable for many heavier organic polymers, which often will decompose long before they sublimate. The process may be summarized as such: (1) The solid is heated by placing it on a boat (made of a high melting point metal) or in a crucible (made of a non-reactive inorganic material) until individual molecules or atoms have enough thermal energy to escape from the solid; (2) the escaped molecules/atoms travel from the source to the target; (3) the molecules/atoms deposit on the substrate, creating a film. Clearly, the evaporation process must be done in a vacuum, so that the evaporated particles can travel from the source to the target without colliding with ambient molecules. It should be noted that both organic semiconductors and metals have the potential to be highly reactive with species such as $\mathrm{O}_{2}$ and $\mathrm{H}_{2} \mathrm{O}$, so such impurities in the deposition chamber may destroy the evaporant, rather than merely scatter it. It is evident that the mean free path of a particle in the deposition chamber be much greater than the distance from the source to the substrate. From [44], the mean free path of an atom in a gas is

$$
\lambda=\frac{1}{\sqrt{2} \rho \pi R^{2}}
$$

where $\rho$ is the density of the gas and $\mathrm{R}$ is the van der Vaals radius of the gas atoms (typically $\sim 1.5 \AA$ ). By applying the ideal gas law, one can quickly rearrange to arrive at:

$$
\lambda=\frac{K_{B} T}{\sqrt{2} P \pi R^{2}}
$$

where $\mathrm{K}_{\mathrm{B}}$ is Boltzman's constant, $\mathrm{T}$ is the temperature, and $\mathrm{P}$ is the pressure. It is seen that the mean free path of a atom in the gas is inversely proportional to the pressure of the gas. Table 3.1 lists the approximate mean free paths of atoms at select pressures. 


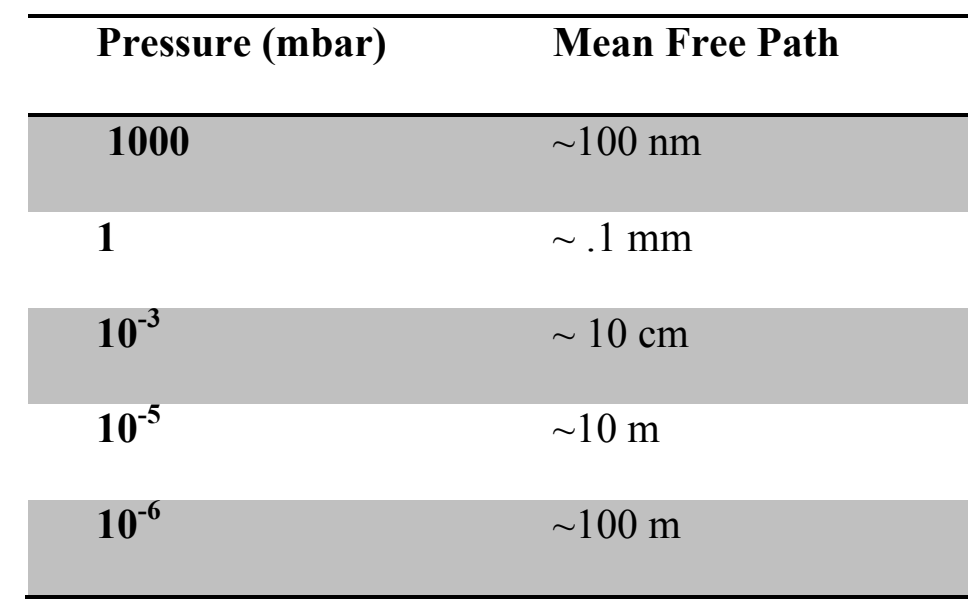

Table 4.1. The mean free path of gas molecules at different pressures.

In a typical deposition chamber the substrate may be many tens of $\mathrm{cm}$ away from the source. Thus, in order to ensure unimpeded travel of the evaporant to the substrate, pressures of $<10^{-5}$ mbar are desired.

In the MBraun thermal evaporation system used in this work, a vacuum of $\sim 10^{-6}$ mbar is obtained via Pfeiffer Vacuum turbomolecular pump (Pfeiffer Vacuum) backed by a rotary vane pump (Edwards), henceforth referred to as the roughing pump. The turbomolecular pump works essentially as a very high-speed turbine selectively imparting momentum to gas molecules driving them towards the exhaust. For proper operation, gas molecules must have a free path in between being impacted by a rotor and being redirected by the subsequent stator. Thus the mean free path of the gas molecules should be greater than the distance of separation of blades in the turbine. Since machining limitations require that the blades be separated by at least a few millimeters, it can be inferred from table 4.1 that the turbomolecular pump will only operate when exhaust pressures are $\sim 10^{-1}$ mbar. The roughing pump is thus required to back the turbomolecular pump by maintaining the suitable exhaust pressure. Evacuation was begun in the MBraun 
system by using the roughing pump to pump the chamber down to $\sim 200$ mbar at which point the turbomolecular pump was started. The turbomolecular pump did not accelerate appreciably until the roughing pump had pumped the chamber down to $10^{-1} \mathrm{mbar}$. At this point the turbomolecular pump quickly accelerated to its operating speed, evacuating the chamber to its final pressure.

The evaporation chamber itself contains restively heated sources, a substrate holder, and a quartz microbalance thickness monitor. A picture of the chamber is shown in Figure 4.6. There are four positions in which sources can be placed. The source may be a tungsten boat (used for the evaporation of aluminum and gold), a molybdenum boat (used for the evaporation of silver), or a quartz crucible (used for the evaporation of organic semiconductors) nestled by a metal bracket. An SQC-223 co-deposition controller is used to control the power delivered to the source. The controller compares the observed deposition rate (as measured by the quartz thickness monitor) against the desired deposition rate and adjusts supplied power accordingly. The controller can also be made to account for the rate of change in the deposition rate in order to temper adjustments to supplied power. This can be used to minimize oscillations in the deposition rate. Typically, metals such as gold and aluminum (Kurt J. Leskar, 99.99\% purity) were evaporated at a rate $\sim 3 \AA / \mathrm{s}$ to a thickness of $30 \mathrm{~nm}$ for top contacts or a thickness of 80 $\mathrm{nm}$ for gate electrodes. Total time for evacuation and evaporation for metal deposition was $\sim 20$ minutes. Organic semiconductors were evaporated at a gentler rate of $.3 \AA / \mathrm{s}$ to a total thickness of $50 \mathrm{~nm}$. Total time in these cases was $\sim 50$ minutes. 


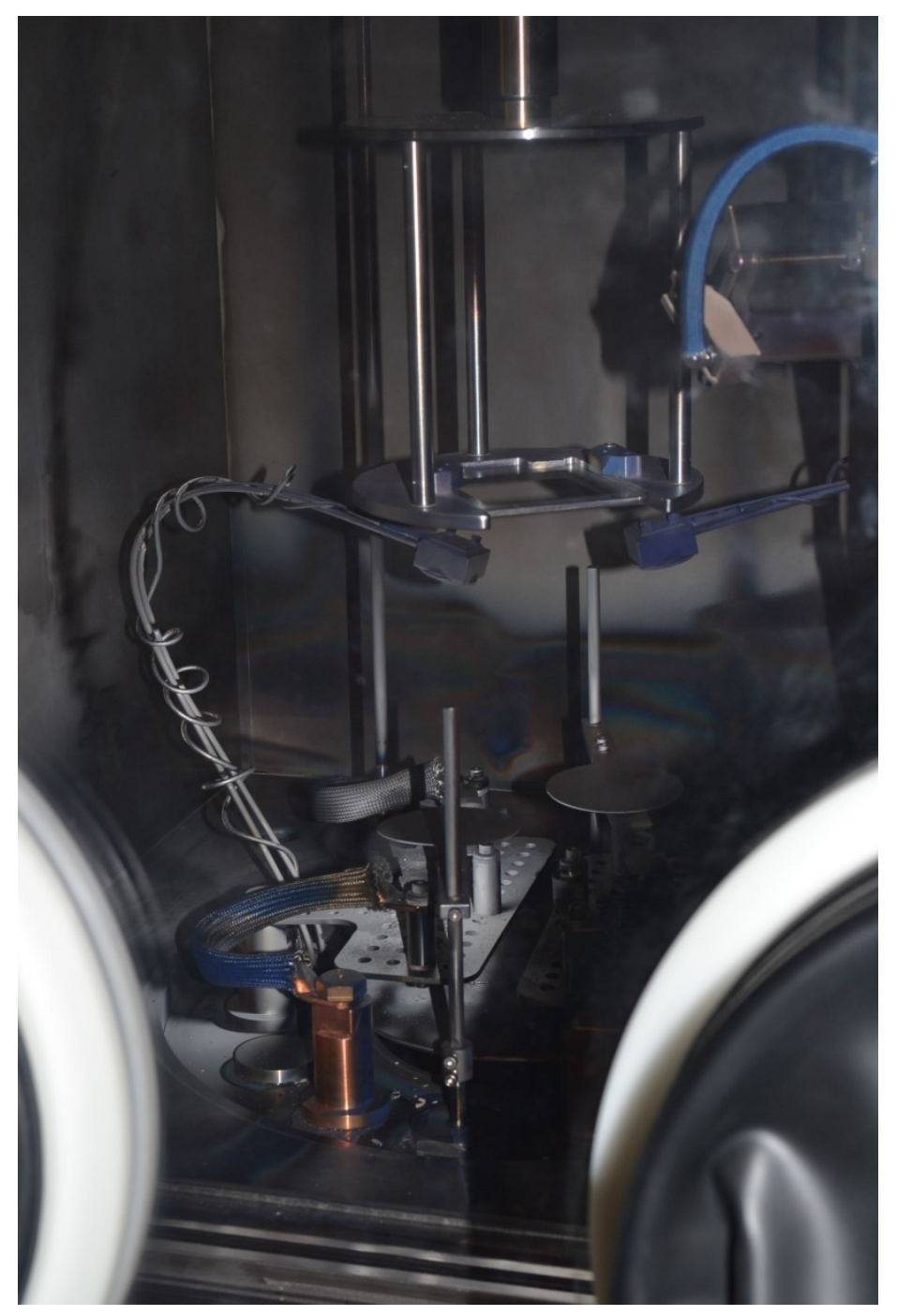

Figure 4.6. Picture of thermal evaporation chamber.

In order to form isolated devices, the metal for the top contacts has to be patterned. The pattern should feature two closely spaced metal contacts separated by a narrow, rectangular region, thus defining the channel. This can be accomplished by evaporating the metal through a shadow mask that has been cut such that it is the negative of the final desired metal pattern. The shadow mask is firmly clamped to the substrate before evaporation to ensure an accurate reproduction of the pattern. Masks made of thin molybdenum were manufactured by JVIC (Missouri State University, Springfield, MO) 
using designs made by the author in the AutoCad software package. A sample mask design is shown in Figure 4.7. The mask resulted in devices with channel lengths of $50-$ $250 \mu \mathrm{m}$ and widths of $.5-2.5 \mathrm{~mm}$, giving $\mathrm{W} / \mathrm{L}$ ratios of 10 to 40 . It may be noted that molybdenum is used for the mask because it has the lowest coefficient of thermal expansion of all common metals ${ }^{45}$ and thus can be cut by laser with minimal distortion of fine features.

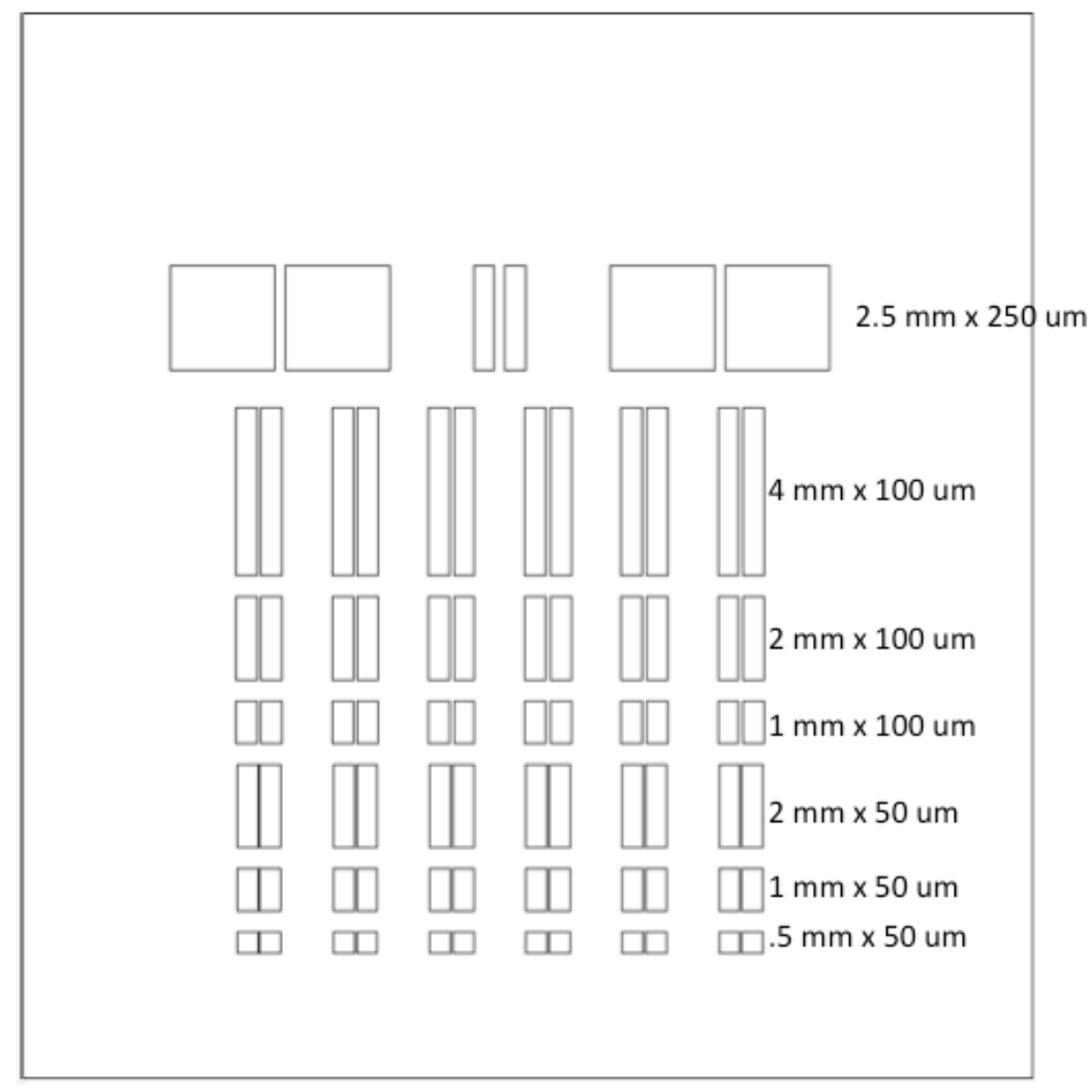

Figure 4.7. Sample mask design used for patterning top contacts for OFETs. The listed to the side of each row give the width and length of the OFET channel. The mask is $1 \mathrm{X} 1$ inch in total size. 


\subsubsection{MAPLE}

MAPLE is a form of pulsed laser deposition (PLD) that allows for the vapor phase deposition of polymeric organic semiconductors. This is in stark contrast to thermal evaporation, which while allowing for the vapor phase deposition of small molecule semiconductors is incompatible with many heavier polymeric semiconductors. PLD itself is a technique whereby a pulsed laser, directed at a solid target, ablates target material into a plume. Atoms or molecules from the plume can then gently deposit onto a substrate. As with thermal evaporation, the deposition must be done in an evacuated environment where vaporized particles have unimpeded travel from the target to the substrate. MAPLE is different from ordinary PLD in that the target is an originally liquid solution that has been made solid by freezing. Thus, in MAPLE the energy incident from the laser may primary be absorbed by the solvent whereas in PLD the bulk material itself must absorb the energy. Thus MAPLE is ideal for materials that may decompose before vaporizing, as is the case for many polymeric semiconductors. Clearly, the laser wavelength must be carefully chosen to ensure that it matches the absorbance of the solvent and is safely separated from the absorbance of the solute.

A diagram of the PLD system used to fabricate devices in this work is shown in Figure 4.8. The entire chamber is evacuated to $10^{-5}$ mbar using a pumping system similar to the one descried in the previous section. The laser is directed though a quartz window to the target, which is mounted on a rotating stage. The target is held in contact with a liquid nitrogen cooled stage, thus ensuring the solvent remains frozen. The substrate is suspended $\sim 4 \mathrm{~cm}$ from the target. 


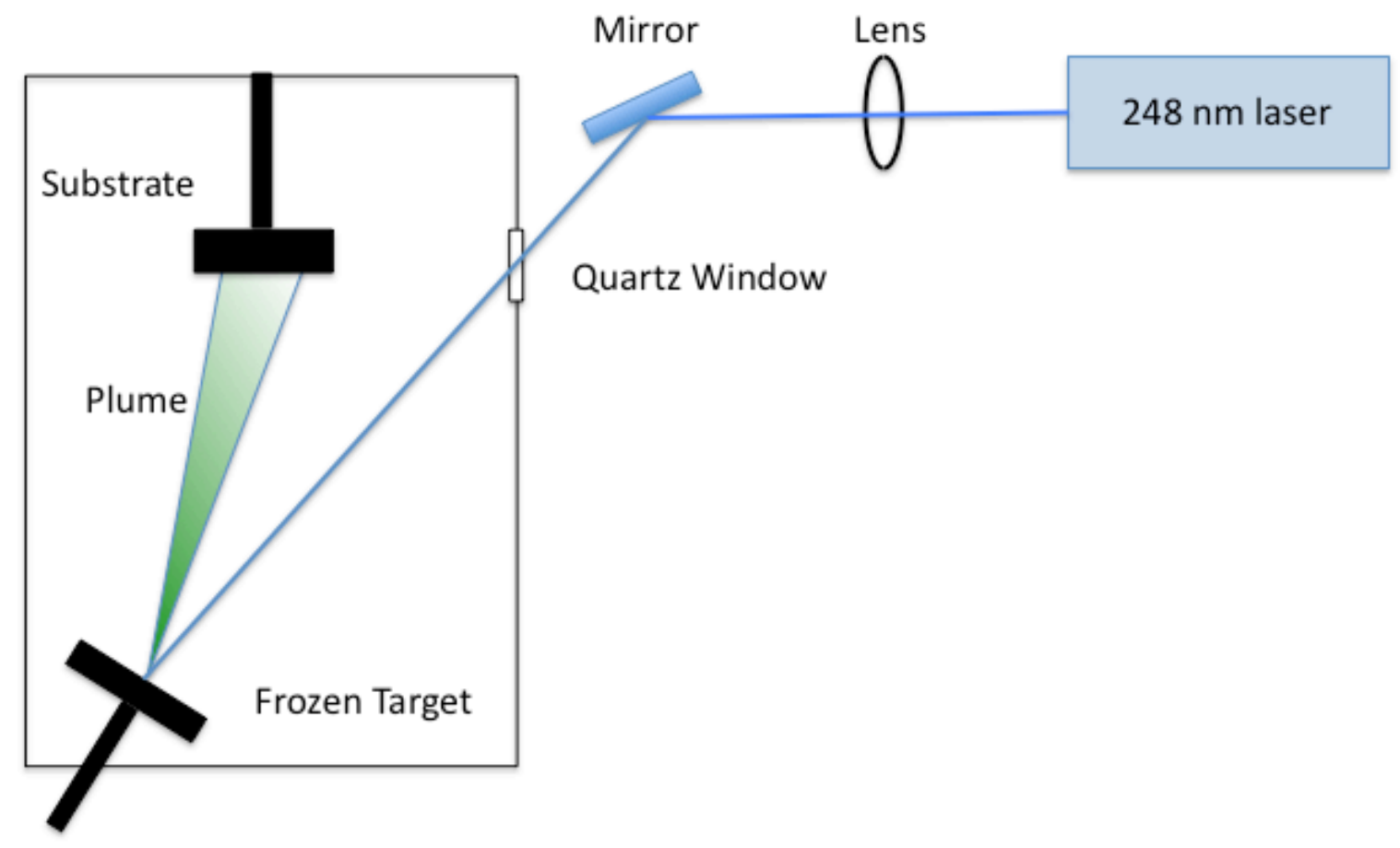

Figure 4.8. Schematic of the PLD system

\subsection{Electrical Characteristics}

After fabrication was completed, it was necessary to ascertain the quality of the OFETs by characterizing their electrical performance. The current-voltage (I-V) characteristics of the devices were measured by using a Keithley 236 Source Measure unit and a Keithley 2440 SourceMeter. As described in section 2.2, a proper characterization will require the sourcing of two voltages (i.e. drain voltage and gate voltage) and the measurement of the respective two currents, so the two units were required to work in tandem. The 2400 has a resolution of $50 \mathrm{pA}$ while the 236 has a resolution of $10 \mathrm{fA}$. The 236 was chosen to measure the drain-source current due to its 
better resolution it offers allowing for the reliable measurement of currents down to $10^{-11}$ A. A schematic of the setup is shown in Figure 4.9.

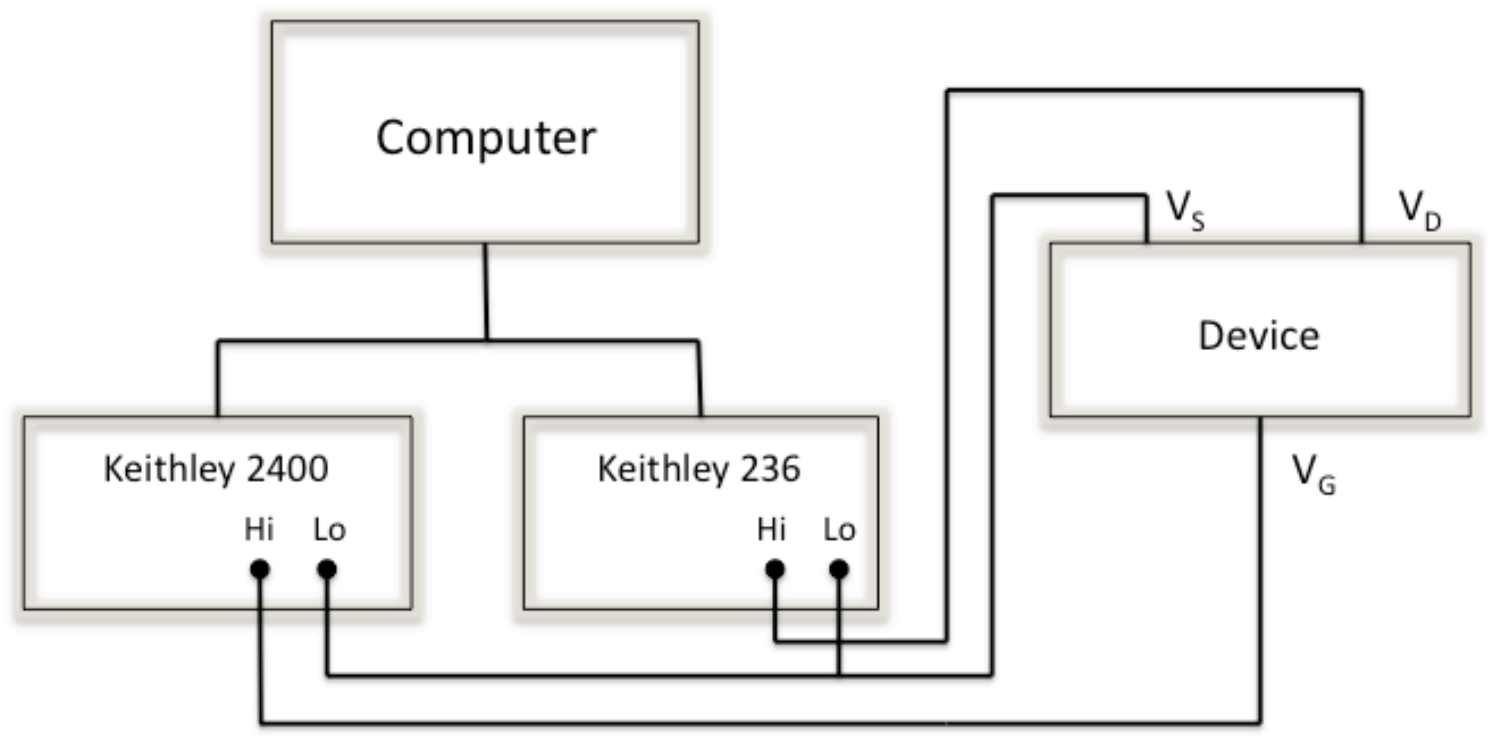

Figure 4.9. Schematic of electrical characteristics testing station

Output characteristics were recorded by writing a LabView (National Instruments) program which set the 2400 to source the constant $V_{G}$ while using a Keithley supplied program to sweep the 236 through the desired $\mathrm{V}_{\mathrm{DS}}$ range as it recorded the current. The program took the desired start and end drain voltage values along with the number of $\mathrm{V}_{\mathrm{DS}}$ points to record and took start and end $\mathrm{V}_{\mathrm{G}}$ points along with number of gate voltage points, and thus allowed for a fully automated way to record a full set of output characteristics.

Transfer characteristics were recorded using a similar program as described above. The 2400 was set to source the $\mathrm{V}_{\mathrm{G}}$ and record gate current while the 236 , once again, sourced $V_{D S}$ and recorded $I_{D S}$. The program was written such that the two units would communicate with the other using triggers such that the 236 would take a drain current 
measurement for each $\mathrm{V}_{\mathrm{G}}$ point sourced by the 2400. Like the output characteristics program, this program allowed for a fully automatic collection of transfer characteristics once the relevant parameters such as start and end voltages and number of points were selected. The program was later modified to automatically extract simple parameters such as charge carrier mobility and threshold voltage from the freshly collected transfer characteristics.

\subsection{Raman scattering measurements}

As discussed in chapter 3, Raman spectroscopy is essentially a very weak inelastic scattering effect. As such, a Raman spectroscopy system must consist of the following parts: (1) an intense monochromatic light source; (2) optics to focus the light source onto the specimen; (3) optics to collect the scattered light; (4) a filter to remove the Rayleigh scattered light; (5) a monchromater to split the Raman scattered light into a continuous spectrum; and (5) a detector to collect the scattered light. It should be noted that, in general, a filter to block the Rayleigh scattered light is not absolutely necessary as a monochromater may suitably split the Raman scattered light from the Rayleigh scattered light. However, the use of such filters can greatly reduce the total space needed for the Raman system. Likewise, while not absolutely necessary, lasers are generally used as the excitation source as they provide the needed intensity and monchromaticity. Furthermore, if the scattered light is collected in a backscattered geometry, a single set of optics may serve to focus and collect the incident and scattered photons, respectively.

All Raman measurements discussed in this work were collected using a Rennishaw InVia Raman Microscope. The system consists of two enclosures, the first of which 
houses the filter, monochromater, and charge coupled device (CCD) detector, and a second, which houses a microscope with a 50X lens that focuses the laser onto the sample and collects the scattered light. A schematic of the system is shown in Figure 4.10. The system has two excitation sources: a $400 \mathrm{~mW} 785 \mathrm{~nm}$ diode laser and a $50 \mathrm{~mW} 514.5$ Argon ion laser. The laser beam enters the enclosure where it is directed to an optical edge filter. The edge filter is chosen with a cutoff wavelength $\lambda$ equal to that of the laser. It completely reflects all wavelengths $\leq \lambda$ and is completely transparent to wavelengths $>$ $\lambda$. Thus, the laser is reflected by the filter and directed through the microscope down onto the sample. The backscattered light is then collected by the microscope and re-redirected onto the filter. Stokes scattered light passes through the filter while the elastically scattered light is blocked. Thus, the filter separates the Raman scattered light from the much more intense laser line. The Raman scattered photons are then passed through a monochromater and focused on a CCD.

The stage on which the sample is placed has automated $\mathrm{x}, \mathrm{y}, \mathrm{z}$ controls, allowing for the point-by-point mapping of the Raman spectrum as a function of position on the sample. Thus, one may place an OFET on to the stage and generate a map of the Raman spectrum as a function of position along the channel and contact regions. The microscope focuses the laser to point $\sim 7 \mu \mathrm{m}$ in diameter so the points may be separated by as little as a few micrometer. The maximum total incident power on sample is $\sim 30 \mathrm{~mW}$ for the $785 \mathrm{~nm}$ laser and $\sim 3 \mathrm{~mW}$ for the $514 \mathrm{~nm}$ laser. 


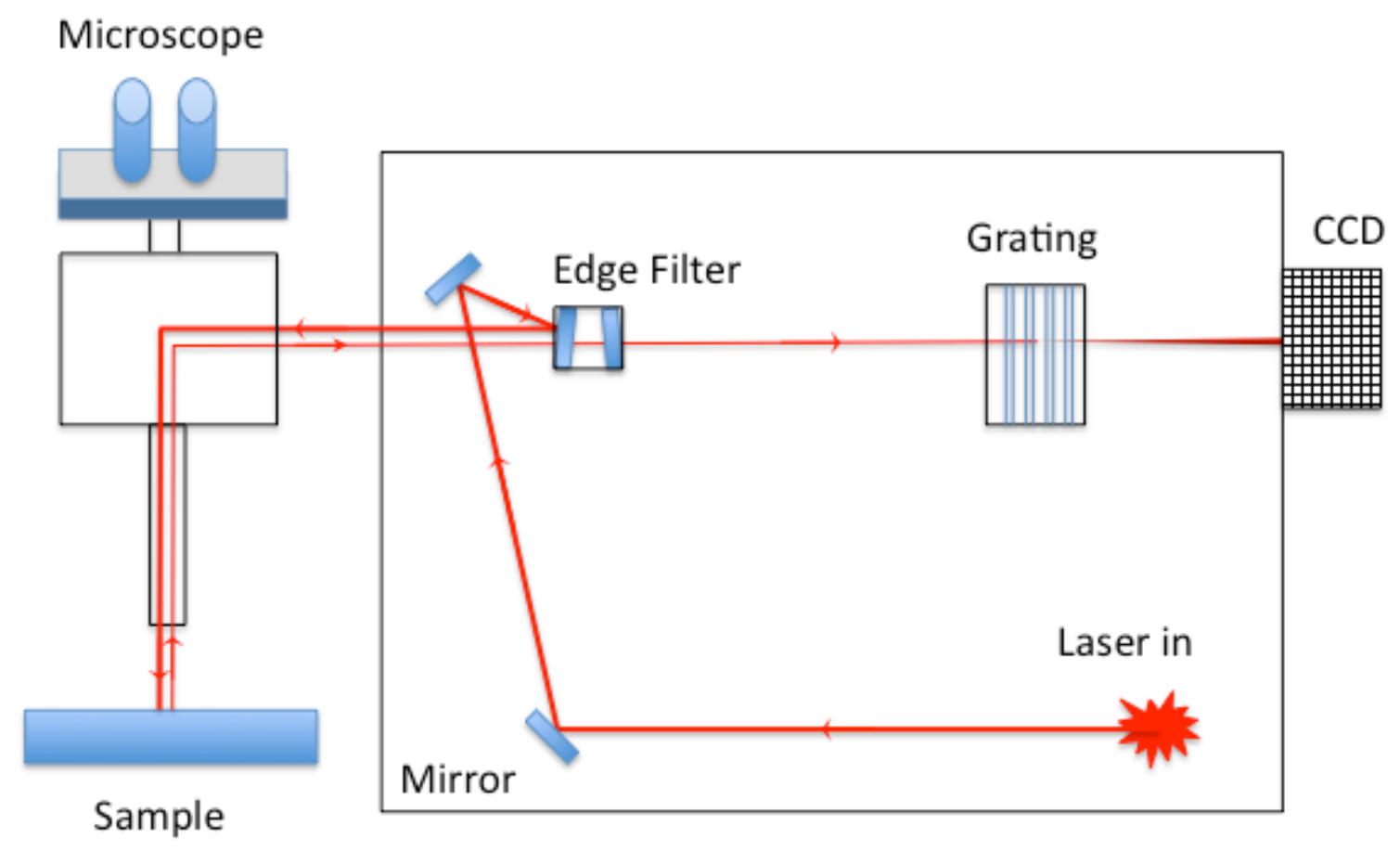

Figure 4.10. Schematic of the micro Raman system. 


\section{Pulsed laser deposited Films for Organic Field Effect}

\section{Transistors}

As mentioned in chapter 2, the performance of OFETs is heavily dependent on the interfaces in the device. The quality of the polymer-dielectric interface, in particular, is determined in large part by the method of forming the polymer film. Spin-coating and dip-coating are typical techniques that are often used to form the film. These methods, however, do not allow for a precise control of the film thickness. ${ }^{46}$ Furthermore, as discussed in chapter 4, if an inorganic dielectric is used, the dielectric surface must be modified by the self-assembly of OTS or other monolayers in order to enhance charge carrier mobilities and device performance.

It would be highly desirable to have an alternate method of polymer film deposition that allows for better film formation without the need for SAM modification of the dielectric. One such method is MAPLE, as discussed in chapter 4. MAPLE allows for the layer-by-layer growth of a homogenous polymer film without any laser-induced damage. ${ }^{47}$ It further allows for the deposition of organic polymer films on solution processable dielectrics. ${ }^{48}$ In general, it is often not possible to deposit such films via spincoating as the dielectric may be susceptible to solvent induced damage. The MAPLE technique has been used to deposit polyfluorene-based, ${ }^{49,50}$ as well as $\mathrm{P} \mathrm{HT}^{51}$ polymer films. The optical and structural properties are similar to that of spin-coated films.

In this work, OFETs were fabricated using a fluorene co-polymer (9,9dioctylfloureneco-bis-N,N-(4-butylphenyl)-bis-N,N-phenyl-1,4phenylenediamine) (PFB), thin film 
grown both by MAPLE and standard spin-coating techniques. Solutions of PFB were prepared for both MAPLE and spin-coating by dissolving the polymer in toluene at concentrations of $2 \mathrm{mg} / \mathrm{ml}$ and $5 \mathrm{mg} / \mathrm{ml}$, respectively, followed by filtering through at $.22 \mu$, PTFE filter. For the MAPLE deposited films, a KrF eximer laser was used as the irradiation source. Toluene is known to have its maximum absorbance at $\sim 260 \mathrm{~nm}$, while the $\mathrm{KrF}$ eximer laser emits at $248 \mathrm{~nm}$. Since the maximum absorbance of PFB occurs at $\sim 350 \mathrm{~nm},{ }^{49}$ energy imparted from the laser is expected to be absorbed by the solvent, and not by the PFB. Furthermore, for polyflourenes films, toluene has been shown to give better films than other Chlorine-bearing solvents. ${ }^{50}$ The laser (Physik COMPex, pulse duration of $20 \mathrm{~ns}$ ), at a pulse rate with an energy density of $0.9 \mathrm{~mJ} / \mathrm{cm}^{2}$, was directed at an angle of incidence of $45^{\circ}$ at the cold target, which had been placed in an evacuated chamber. The target was constantly rotated to avoid heating induced decomposition at a single spot. The growth rate was $.6 \mathrm{~nm} / \mathrm{min}$ and the final film thickness was $\sim 20 \mathrm{~nm}$.

Spun-cast films were deposited by spin-coating (2000 rpm) .3ml of PFB solution on both untreated $\mathrm{SiO}_{2}$ and OTS treated $\mathrm{SiO}_{2}$ substrates. The final thickness of the spun-cast films was $\sim 50 \mathrm{~nm}$. Devices using both the spun-cast and PLD films were completed by depositing the top $\mathrm{Au}$ contacts $(\sim 30 \mathrm{~nm})$ at a rate of $3 \AA / \mathrm{s}$ using thermal evaporation. A shadow mask was used to pattern OFETs with typical channel lengths of $.05-.1 \mathrm{~mm}$ and widths of .5-1mm. MIS structures were likewise patterned to give device areas of $2.2 \mathrm{X}$ $10^{-3} \mathrm{~cm}^{2}$.

Here, we compare the FET and MIS characteristics of the devices made from the spun-cast and MAPLE grown films. We use I-V measurements from the OFETs and capacitance-voltage (C-V) and conductance-voltage (G-V) measurements from the MIS 
structures to construct a detailed analysis of the polymer-dielectric interface The electrical characteristics of all the devices are compared to show that without any OTS treatment of the $\mathrm{SiO}_{2}$ surface, MAPLE grown OFETs show better transistor performance than the spin-coated deposited OFETs.

Both MAPLE grown and spin-coated films were deposited on OTS and non-OTS treated $\mathrm{SiO}_{2}$. Figures 5.1 and 5.2 show the transfer characteristics from MAPLE-grown and spin-coated PFB-based OFETs on non-OTS treated $\mathrm{SiO}_{2}$, respectively. The insets show the output characteristics. The spin-coated sample shows a higher leakage current and a lower modulation of the drain-source current by the gate voltage. Device parameters such as charge carrier mobility, On/Off current ratio, and threshold voltage were estimated using the standard saturation regime current-voltage characteristics of field-effect transistors (eq. 2.7).

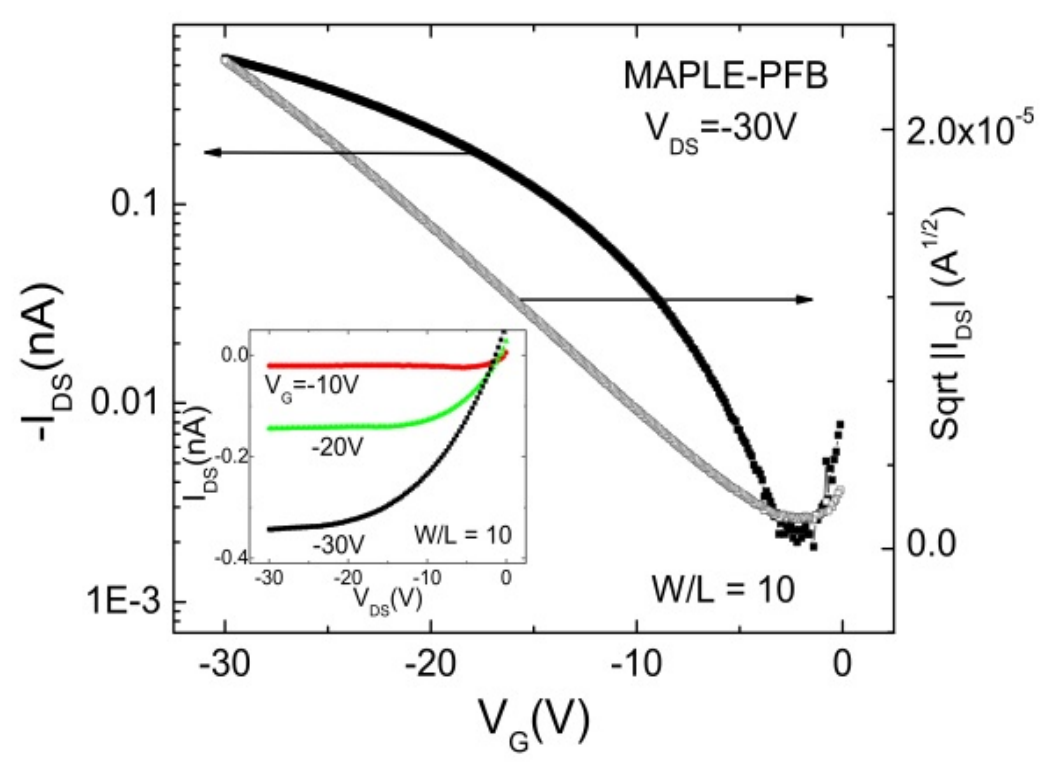

Figure 5.1. Transfer characteristics of MAPLE grown PFB-OFET on a non-OTS substrate at a drain-source voltage of $-30 \mathrm{~V}$. The inset shows the output characteristics. 


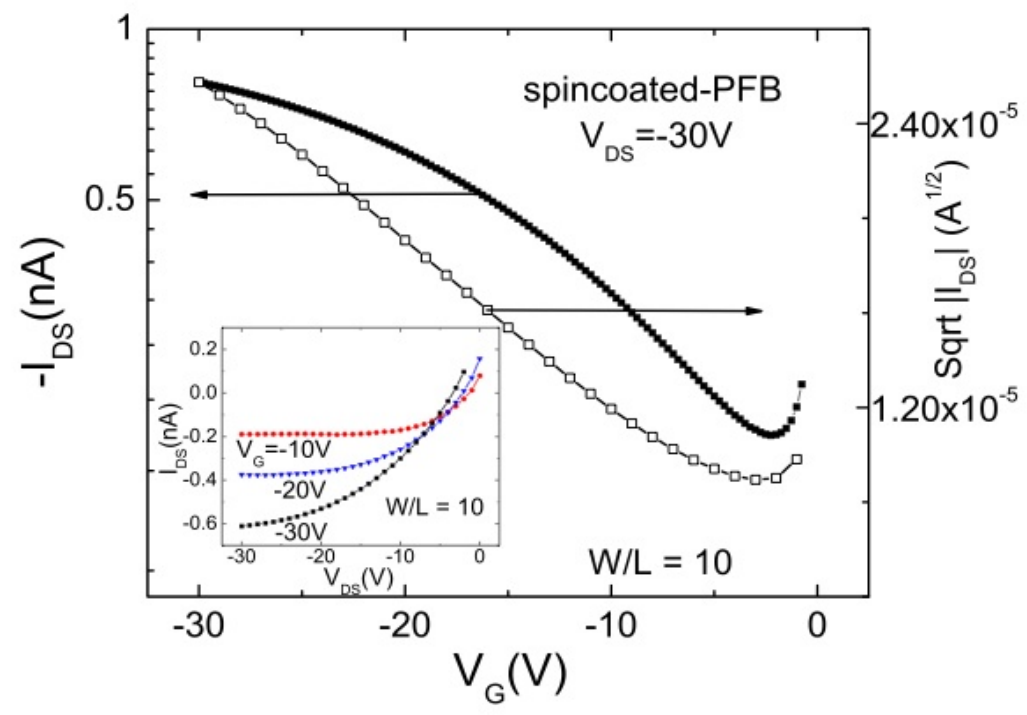

Figure 5.2 Transfer characteristics of spun-cast PFB-OFET on a non-OTS substrate at a drain-source voltage of $-30 \mathrm{~V}$. The inset shows the output characteristics.

Transistor characteristics for MAPLE-grown and spin-coated PFB-based OFETs on OTS treated $\mathrm{SiO}_{2}$ are shown in figures 5.3 and 5.4, respectively. The device structure and size were identical for the MAPLE grown and spin-coated OFETs. The hole mobility is deduced from a linear fit to the saturation region of the square root of the drain-source current as a function of the gate voltage (figures 5.1, 5.2, 5.3, and 5.4). The results are tabulated in table 5.1. We point out that due to the amorphous nature of the polymer, the hole mobilities are inherently lower compared to semicrystalline polymers such as F8T2 and $\mathrm{P} 3 \mathrm{HT}^{52,53}$.

While the OTS treated spin-coated films perform significantly better than their nonOTS treated counterpart, the OTS treated MAPLE devices show only slightly better performance than their non-OTS treated counterparts. The On/Off ratio is consistently 
better for the MAPLE grown PFB OFETs since these devices have lower off currents. Since $\mathrm{V}_{\mathrm{Th}}$ is typically independent of morphology ${ }^{54}$, the apparent positive $\mathrm{Vth}$ is attributed to the relatively high off currents in the spun-cast samples. To obtain further insight into the nature of the polymer-dielectric interface, capacitance-voltage measurements were carried out form the MIS structures.

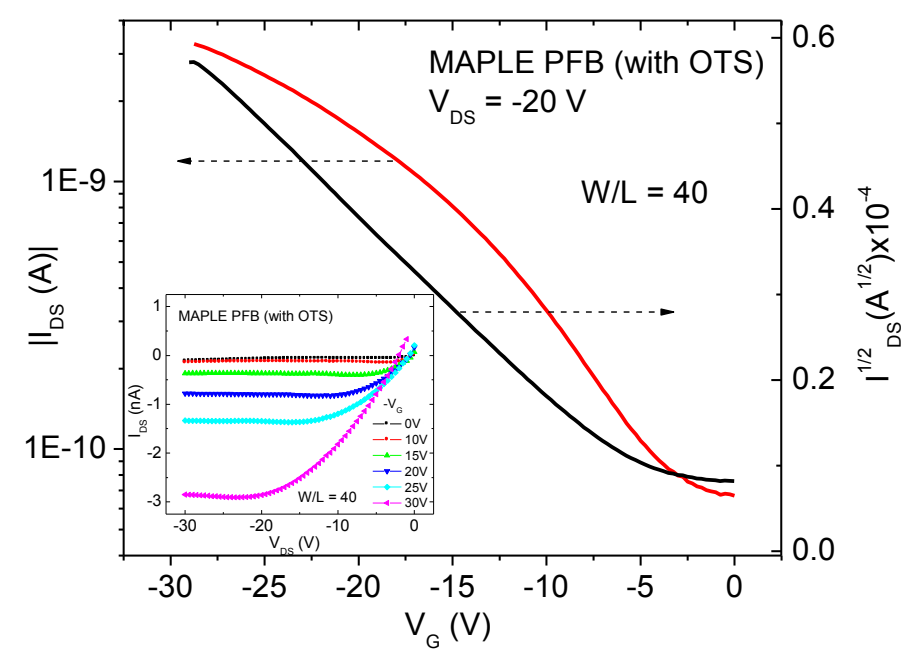

Figure 5.3. Transfer characteristics of MAPLE deposited PFB-OFET on an OTS treated substrate at a drain-source voltage of $-20 \mathrm{~V}$. The inset shows the output characteristics 


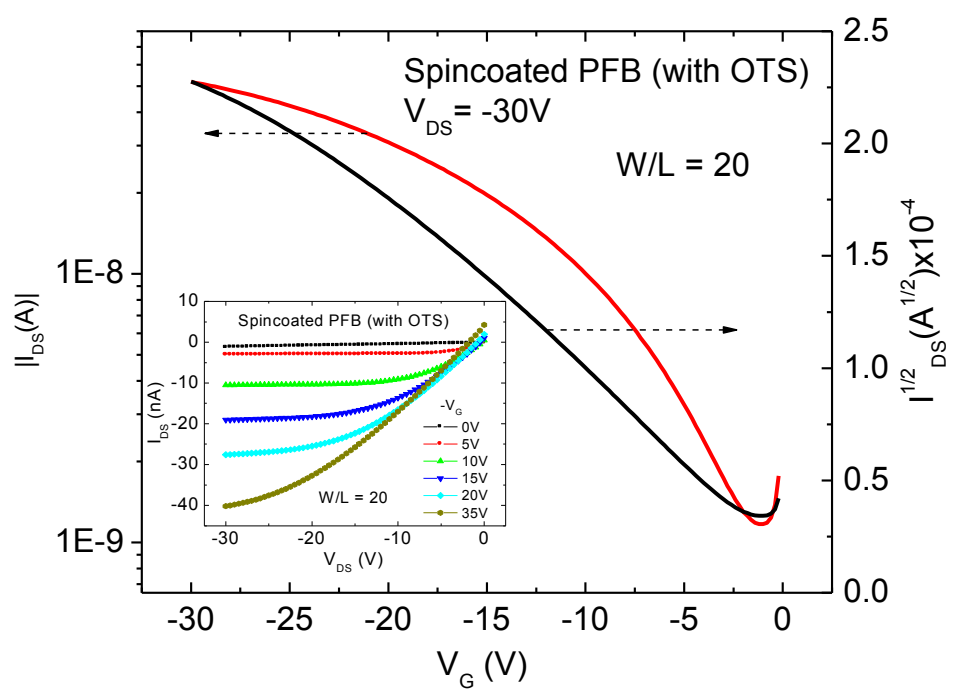

Figure 5.4. Transfer characteristics of spun-cast PFB-OFET on an OTS treated substrate at a drain-source voltage of $-30 \mathrm{~V}$. The inset shows the output characteristics

Table 5.1. OFET characteristics of MAPLE grown and spincoated films on non-OTS and OTS modified $\mathrm{SiO}_{2}$.

\begin{tabular}{lllll}
\hline & Spun-cast & Spun-cast OTS & MAPLE & MAPLE OTS \\
\hline $\begin{array}{l}\text { Mobility } \\
\left(\mathbf{c m}^{2} / \text { Vs }\right)\end{array}$ & $3 \times 10^{-6}$ & $4 \times 10^{-4}$ & $8 \times 10^{-6}$ & $2 \times 10^{-5}$ \\
$\begin{array}{l}\text { On/Off Ratio } \\
\text { Threshold }\end{array}$ & 10 & 30 & 200 & 250 \\
Voltage $\left(\mathbf{V}_{\mathbf{T h}}\right)$ & 6 & 2 & -2 & -2 \\
\hline
\end{tabular}

Figure 5.5 shows the $\mathrm{C}-\mathrm{V}$ characteristics of the spun-cast and MAPLE grown PFB diodes measured in the frequency range of 2-6 kHz. The insets show their hysteresis behaviors at a frequency of $2 \mathrm{kHz}$. Both of these samples were fabricated on OTS treated substrates. Both samples show high capacitance measured in the accumulation region at negative bias voltages and low capacitance measured in the depletion region at positive 
bias voltages. The magnitude of the hysteresis is $\sim 14.3 \mathrm{~V}$ and $24.4 \mathrm{~V}$ for the spun-cast and MAPLE grown samples, respectively. Both samples exhibit frequency dispersions in the accumulation region, with the spin-cast devices showing a slightly higher dispersion.
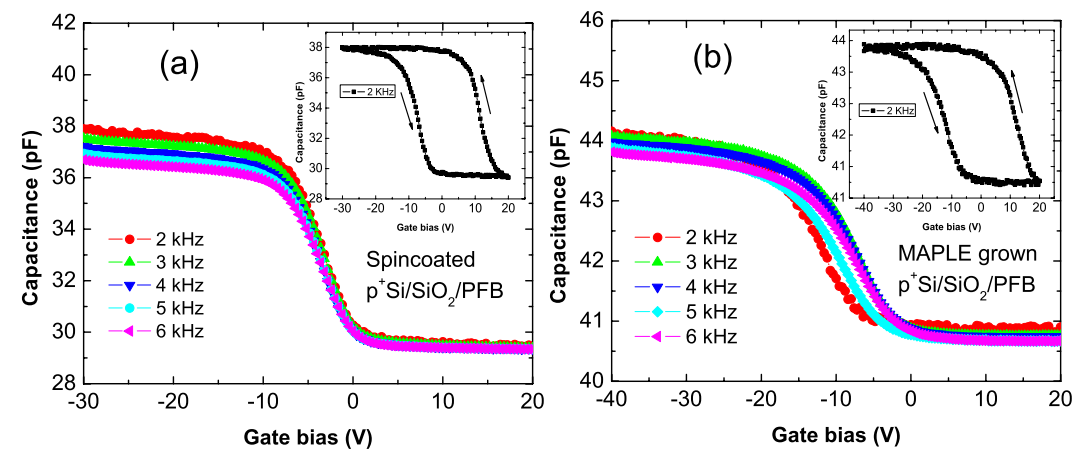

Figure 5.5. Frequency dependence of the $\mathrm{C}-\mathrm{V}$ curves for (a) spun-cast $\mathrm{p}^{++}$$\mathrm{Si} / \mathrm{SiO}_{2} / \mathrm{PFB}$ and (b) MAPLE grown $\mathrm{p}^{++}-\mathrm{Si} / \mathrm{SiO}_{2} / \mathrm{PFB}$ diodes. The insets show the hysteresis at $2 \mathrm{kHz}$. The arrows denote the direction of the sweep cycles.

The extent of the frequency dispersions in the accumulated capacitance are $\sim 3.4 \%$ and $0.6 \%$ respectively, which is considerable less than reported for some MIS structures. ${ }^{55,56}$ The observed frequency dispersions in the accumulation capacitance could possible be due to mobile charges acting as trapped charges at higher frequencies due to their nonresponse to such high frequencies. The MAPLE grown sample shows a slightly larger stretching of the $\mathrm{C}-\mathrm{V}$ curve along the voltage axis as well as frequency dispersion in the depletion region, which may be accounted for by the slightly higher interface trap density $\left(D_{i t}\right)$ values estimated in this sample. ${ }^{57}$ 
Though the spun-cast sample showed slightly lower $D_{i t}$ values, the MAPLE grown sample exhibited less accumulation capacitance and time constant dispersions. While energy loss in the MAPLE grown sample is dominated by interface trap states, energy loss in the spun-cast sample is dominated by bulk states, with some interface states contribution. Overall it is seen that MAPLE grown MIS structures perform similar to the spun-cast ones, even though the surface roughness of the former is higher. ${ }^{49}$

In conclusion, this work has shown the MAPLE technique to be a viable alternative to spin-coating in the fabrication of device-quality organic MIS diodes and FETs. In MIS structure though the spun-cast sample showed slightly lower $D_{i t}$ values, the MAPLE grown sample exhibited less accumulation capacitance and time constant dispersions. The FETs show a consistently better on/off ratio for the MAPLE grown PFB films compared to the spun-cast films. MAPLE grown films provide an added advantage of patterning the active layer with minimum surface modification requirements of the dielectric-polymer interface. This opens up potential applications of the MAPLE technique in nanostructured organic devices. 


\section{Light scattering of Studies of Pentacene}

In addition to the dielectric-semiconductor interface, the semiconductor- metal interface plays a crucial role in the performance of an OFET. There have been a few investigations of the interfaces of organic molecules with metals such as $\mathrm{Ag}, \mathrm{Al}$, and $\mathrm{Mg}$ using Raman spectroscopy. Most of these studies involved vacuum evaporated thin metal films. Depending on the choice of the metal, the Raman spectrum of organic semiconductors show an enhancement of intensities due to the SERS effect, ${ }^{58}$ changes in relative intensities due to complexes formed by weak interaction between the metal and the conjugated ring of the ligand, ${ }^{59}$ and also partial graphitization of the semiconductor film. ${ }^{60}$

The nanostructured size and shape of the metal layer on top of the organic films allows for the SERS effect. Since most p-type OFETs utilize Au contact electrodes, a question that may be raised is whether SERS, if observable, may be used as a diagnostic tool to probe the organic semiconductor-metal interface in terms of its electronic and chemical makeup, along with providing a platform to understand any degradation related mechanisms in OFET performance.

Here, we explore the interface between Au and pentacene using Raman spectroscopy by varying the thickness of the Au layer in pentacene-based OFET structures. With a near infrared (IR) excitation source, SERS from the pentacene layer is clearly seen beyond a certain thickness of Au. Raman maps across the pentacene-Au interface provide a strong visualization tool to study the impact of the metal layer on the pentacene film. This

technique further provides insights into device performance and defect-induced 
mechanisms when gated. It is seen that the SERS spectrum detects molecular degradation in high-operating voltage devices. This is contrasted with low-operating voltage devices, where no such degradation is detected. The pentacene results are also compared with crystalline graphite, where Au evaporation results in an SERS effect enhancing the features from disordered carbons.

A comparison of the experimental results with first-principles vibrational frequency calculations of pentacene clearly show that $\mathrm{Au}$ deposition results in a change in the aromaticity of the phenyl rings and introduction of disordered carbons. The Raman signature of these disorder pentacene molecules is further enhanced by SERS. In fact, it is shown that it is possible to simulate the SERS spectrum by explicitly modeling the Aupentacene interaction. The thermal evaporation of the pentacene film itself retains the aromaticity and structure of the molecule, evident from the Raman spectrum itself.

Finally, the SERS effect from pentacene films covered with evaporated Ag films is investigated. It is shown that with both $\mathrm{Au}$ and $\mathrm{Ag}$, the SERS spectrum evolves as a function of metal film thickness. In fact, the spectrum shows sub-nanometer level sensitivity to the thickness of the metal film for very thin films. Thus, a Raman scattering based metrology tool for tracking the thickness of top metal contacts on organic films is proposed.

\subsection{Observation of SERS}

Figure 4.1 shows the representative Raman spectra of a pentacene film in the 1100 $1700 \mathrm{~cm}^{-1}$ region from the channel region and through the Au pad of an OFET using the $785 \mathrm{~nm}$ laser line as the excitation source. The Au thickness of this device was $5 \mathrm{~nm}$. A 
detailed description of the Raman frequencies of pentacene is found in Ref. $[61,62,63]$. The Raman peaks in the $1140-1190 \mathrm{~cm}^{-1}$ region originate from the C-H in-plane bending motion, and the C-C aromatic stretching vibration lies in the $1340-1400 \mathrm{~cm}^{-1}$ range. The two peaks at $\sim 1160 \mathrm{~cm}^{-1}$ and $1178 \mathrm{~cm}^{-1}$ are related to the motion of the $\mathrm{H}$ atoms at the end and the side of the molecule. The $1371 \mathrm{~cm}^{-1}$ peak is seen as the strongest $\mathrm{C}-\mathrm{C}$ stretching vibration.

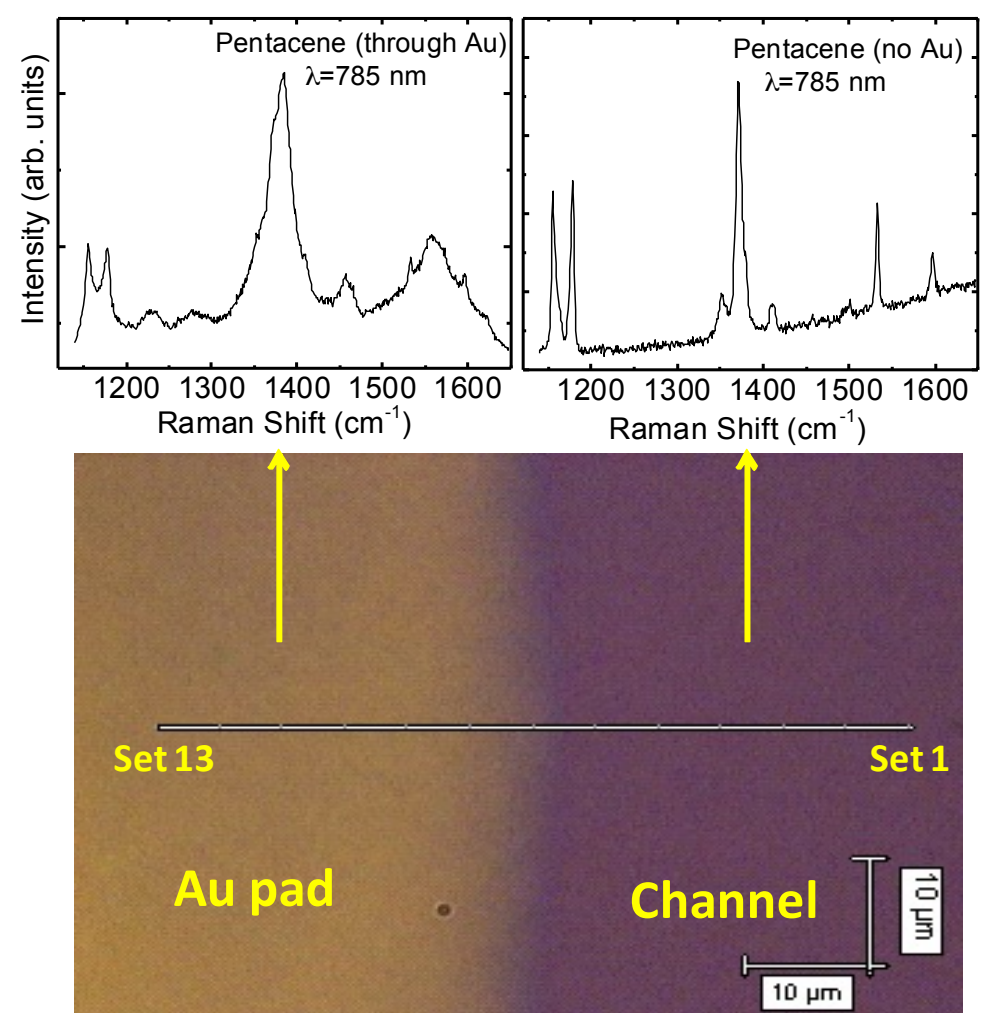

Figure 6.1. Optical image of the channel/contact region of a pentacene-based OFET. The Raman spectrum of pentacene in the $1120-1600 \mathrm{~cm}^{-1}$ is shown on top through the $\mathrm{Au}$ pad (left) and from the channel region (right).

It can quickly be seen from Figure 6.1 that the $1380 \mathrm{~cm}^{-1}$ and the $1580 \mathrm{~cm}^{-1}$ region are broadened with the appearance of new peaks when probed through the Au pad. 
Additionally, the intensity of the $1380 \mathrm{~cm}^{-1}$ peak is greatly enhanced through $\mathrm{Au}$, which is not seen here as the two spectra are normalized to the intensity of the $1380 \mathrm{~cm}^{-1}$ peak. The relative intensities of the $1160 \mathrm{~cm}^{-1}$ and $1178 \mathrm{~cm}^{-1}$ Raman peaks also change with the thickness of the Au film. To understand the role of $\mathrm{Au}$ in the Raman scattering of pentacene, we have systematically varied the thickness of the Au layer and measured the Raman spectrum from the channel-Au pad region using both the $785 \mathrm{~nm}$ and the $514 \mathrm{~nm}$ excitation sources.

The largest SERS enhancement due to Au nanoparticles/nanostructures occurs when excitation wavelengths are in the near infrared, out of resonance with the surface plasmons.$^{64}$ Comparing the Raman spectrum of pentacene through the Au pad using both the $785 \mathrm{~nm}$ and the $514 \mathrm{~nm}$ further distinguishes the SERS effect from normal Raman scattering. Figure 6.2 shows the Raman spectra of pentacene films (of same thickness) in the $1150 \mathrm{~cm}^{-1}-1600 \mathrm{~cm}^{-1}$ region for three different Au layer thicknesses measured with the $785 \mathrm{~nm}$ excitation. No changes are observed in the Raman spectrum of pentacene for Au film thickness $<1 \mathrm{~nm}$. As the Au thickness increases, new peaks at $1381 \mathrm{~cm}^{-1}$ and $1561 \mathrm{~cm}^{-1}$ emerge, as seen in Figure 6.1. For typical Au thicknesses used in devices (> 20 $\mathrm{nm})$, these two peaks broaden and dominate the Raman spectrum. Moreover, the intensity of the $1380 \mathrm{~cm}^{-1}$ region keeps increasing with the Au layer thickness and saturates at about $10 \mathrm{~nm}$. 


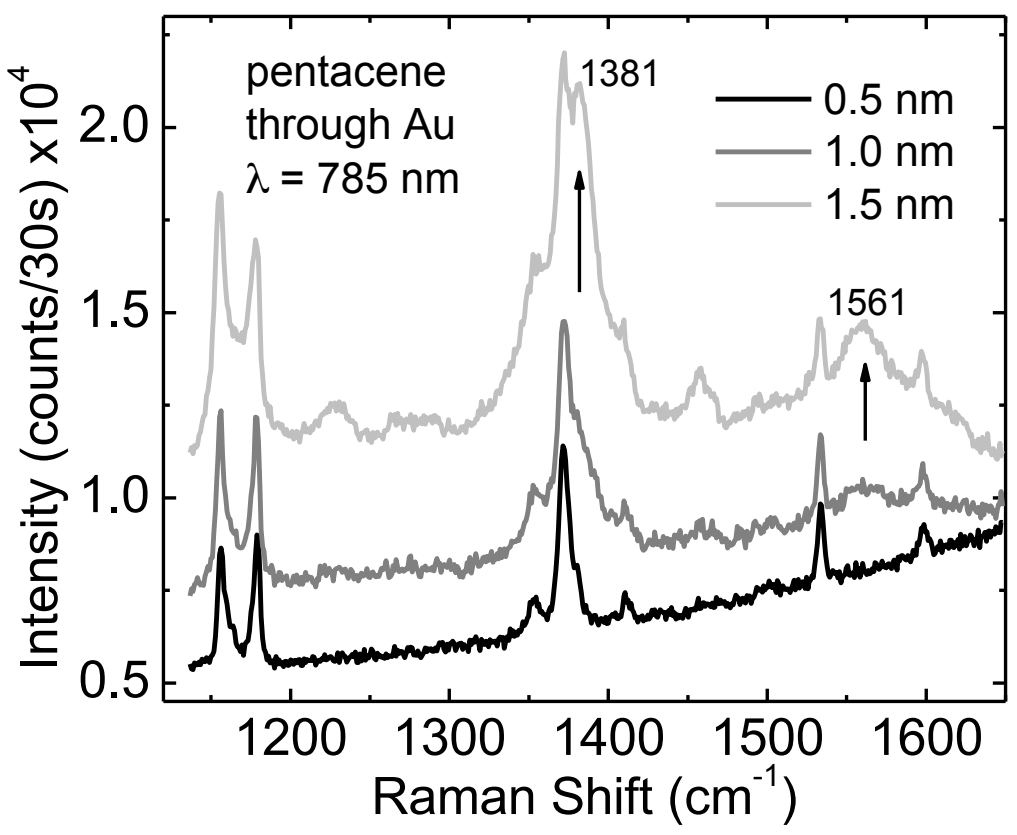

Figure 6.2. The Raman spectra of pentacene films covered with Au films of three different thicknesses.

\subsection{Confirmation of SERS effect}

To understand the origin of the new features observed in the Raman spectrum of pentacene with increasing thickness of the Au layer, several of these films were measured with the $514 \mathrm{~nm}$ laser line. Figure 6.3 (a) shows a schematic of the setup, where either the $785 \mathrm{~nm}$ or the $514 \mathrm{~nm}$ line were incident on the sample in a backscattering geometry. Figure 6.3 (b) and (c) show the Raman spectra of pentacene measured with and without the Au layer using the $785 \mathrm{~nm}$ and $514 \mathrm{~nm}$ lasers, respectively. Both sets of spectra were measured from the same region in the sample that had a $20 \mathrm{~nm}$ Au layer. The Raman spectrum of pentacene through $\mathrm{Au}$ with the $785 \mathrm{~nm}$ line is greatly enhanced compared to 
pentacene only signal, as evident from Figure 6.3 (b). The $1380 \mathrm{~cm}^{-1}$ and the $1560 \mathrm{~cm}^{-1}$ peaks dominate and completely overwhelm the other pentacene Raman peaks in this region. Qualitatively the Raman spectrum measured with the $514 \mathrm{~nm}$ line through $\mathrm{Au}$ is similar without $\mathrm{Au}$ except for the shoulders at $1390 \mathrm{~cm}^{-1}$ and $\sim 1570 \mathrm{~cm}^{-1}$, denoted by the dotted lines. The overall CCD counts through the Au layer is lower compared to the pentacene only signal due to attenuation of the green laser light. Clearly, there is no enhancement of the Raman signal when the $514 \mathrm{~nm}$ laser is used. Since this laser has an energy that is far away from that of the Au surface plasmons $(>1 \mathrm{eV})$, it will not be able to excite them. Therefore, the absence of any enhancement when using the higher energy laser confirms that the enhancement seen with the $785 \mathrm{~nm}$ source is due to excited $\mathrm{Au}$ surface plasmons and thus, is indeed an SERS effect. 

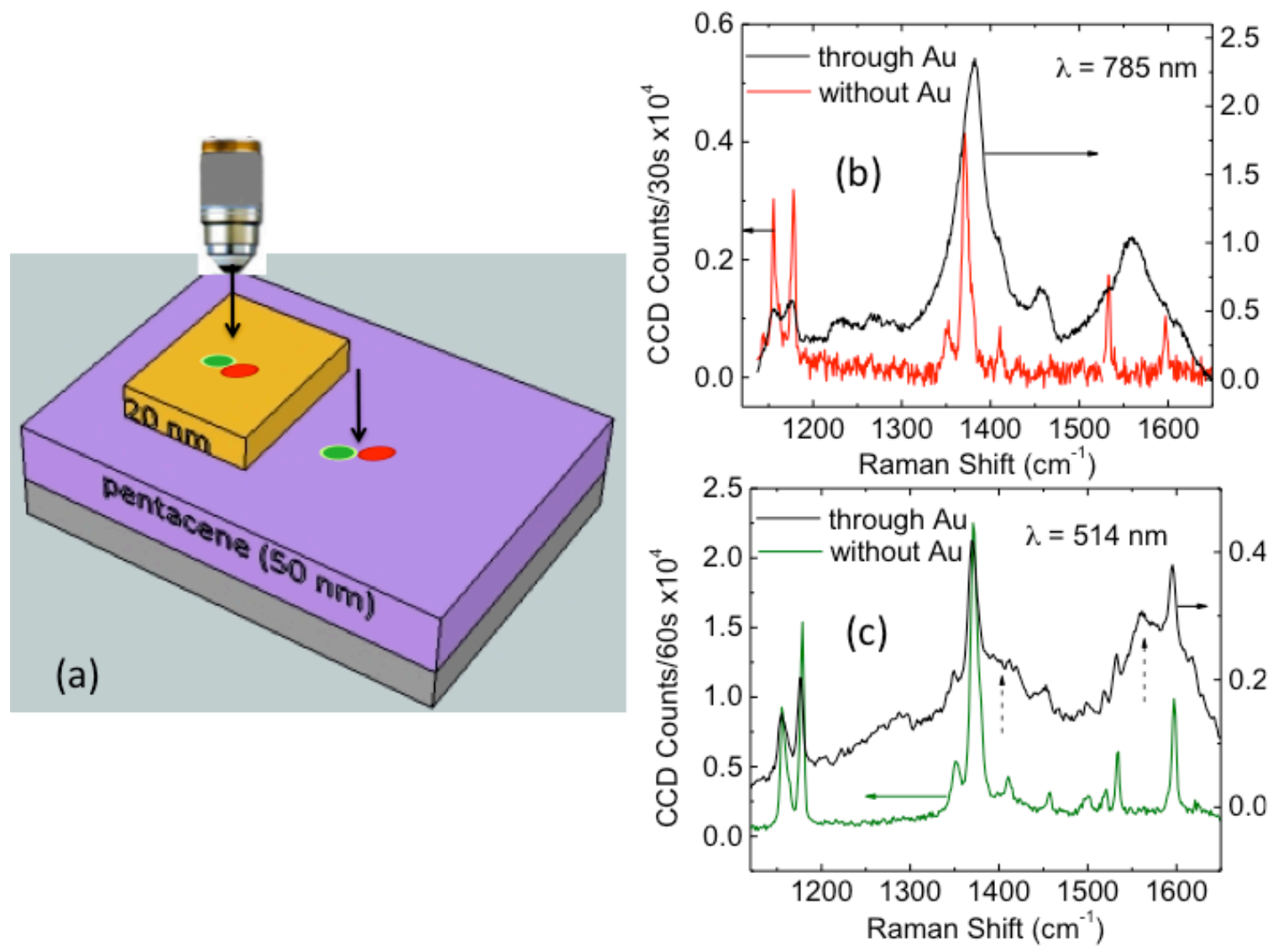

Figure 6.3. a) Schematic of the Raman setup in a backscattering geometry. (b) The Raman spectra from two regions in the sample - through a $20 \mathrm{~nm}$ thick Au layer and without any Au layer with the $785 \mathrm{~nm}$ excitation source. (c) The Raman spectra from the same regions as in (b) but measured with the $514 \mathrm{~nm}$ laser line.

As a further check we also evaporated a thin layer of $\mathrm{Au}(10 \mathrm{~nm})$ on a piece of highly oriented pyrolytic graphite (HOPG), and measured the Raman spectrum both with the $785 \mathrm{~nm}$ and the $514 \mathrm{~nm}$ excitation sources. HOPG consists of a continuous, ordered $\mathrm{sp}^{2}$ carbon network, similar to that of pentacene. With the $514 \mathrm{~nm}$ excitation, no changes are observed in the Raman spectrum of HOPG when probed through the Au film or without Au. However, the $785 \mathrm{~nm}$ excitation source shows features at $1306 \mathrm{~cm}^{-1}$ and $1540 \mathrm{~cm}^{-1}$ (Figure 6.4). The $1306 \mathrm{~cm}^{-1}$ is clearly the D-band of disordered graphite. The enhanced 
sensitivity of the Raman spectrum when using the $785 \mathrm{~nm}$ excitation source is only explainable by there being a surface enhancement at the Au-graphite interface. Thus, we see that the enhancement is not an artifact of the Au-pentacene systems, but is truly a surface effect based on the interaction of the $785 \mathrm{~nm}$ excitation source with the Au layer surface.

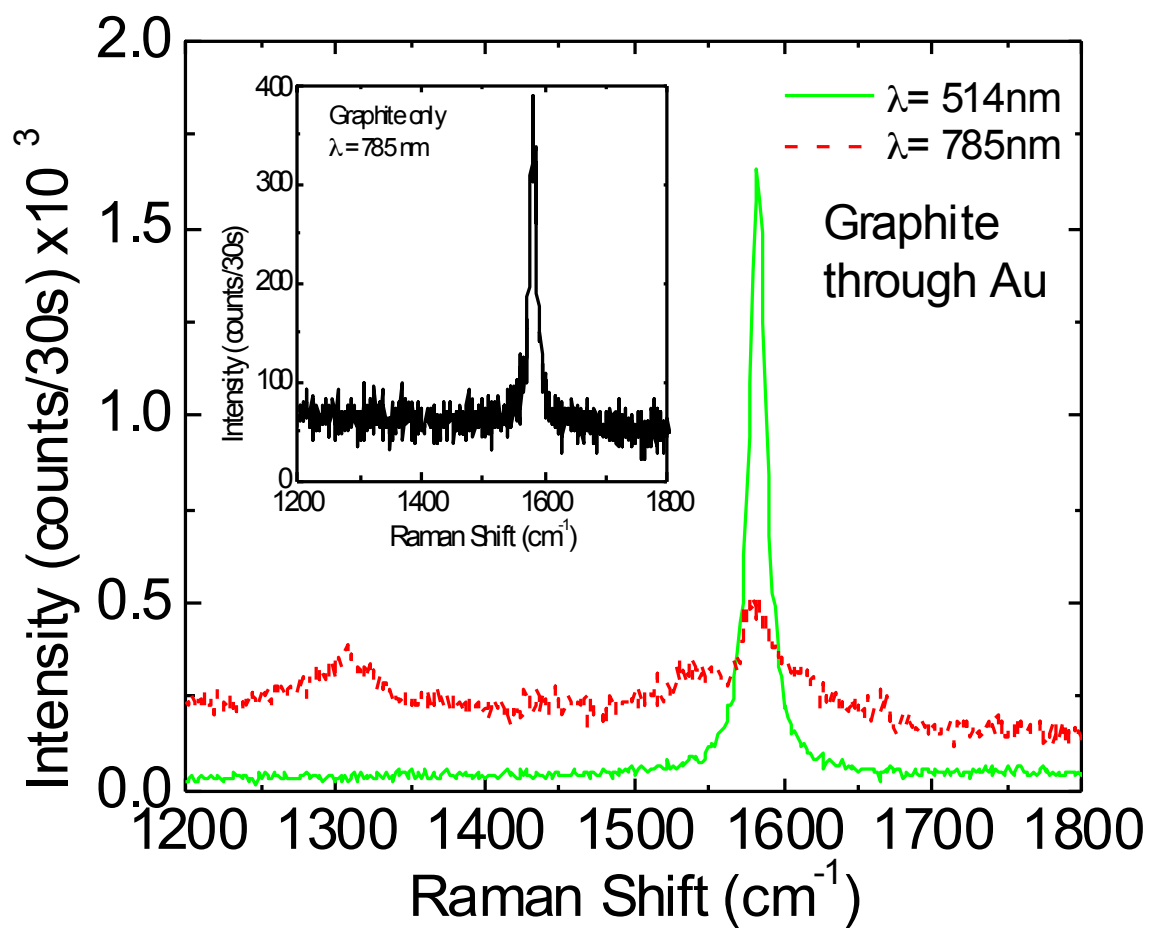

Figure 6.4. Raman spectra of graphite through a thin Au film using the $785 \mathrm{~nm}$ and $514 \mathrm{~nm}$ excitation sources. The inset shows the Raman spectrum measured with the 785 nm line without any $\mathrm{Au}$.

Another way of confirming that there is a surface plasmon based effect at the heart of the enhancement is to directly measure the absorbance of the pentacene-Au interface. Au surface plasmons are known to emit in the near infrared portion of the spectrum and absorb $\sim 540 \mathrm{~nm}$. If there are surface plasmons that are capable of creating the SERS 
effect at the interface, then they should be apparent in the absorption spectrum of the interface. A very thin layer of $\mathrm{Au}(\sim 2 \mathrm{~nm})$ was deposited onto a cleaned glass slide. As a last check, we measured the absorbance of the $\sim 2 \mathrm{~nm}$ film of $\mathrm{Au}$ by itself. Upon deposition, Au has the tendency to form nano-islands, which then merge to form the film. Thus, the $\mathrm{Au}$ film on glass will also have a similar nano-roughness, as does the $\mathrm{Au}$ surface in the Au-pentacene interface. The absorbance is shown in Figure 6.5. Here, the absorbance of the surface plasmons is seen clearly. We, therefore, have very strong, direct evidence that there are surface plasmons at the Au surface, giving yet further support to the existence of the SERS effect.

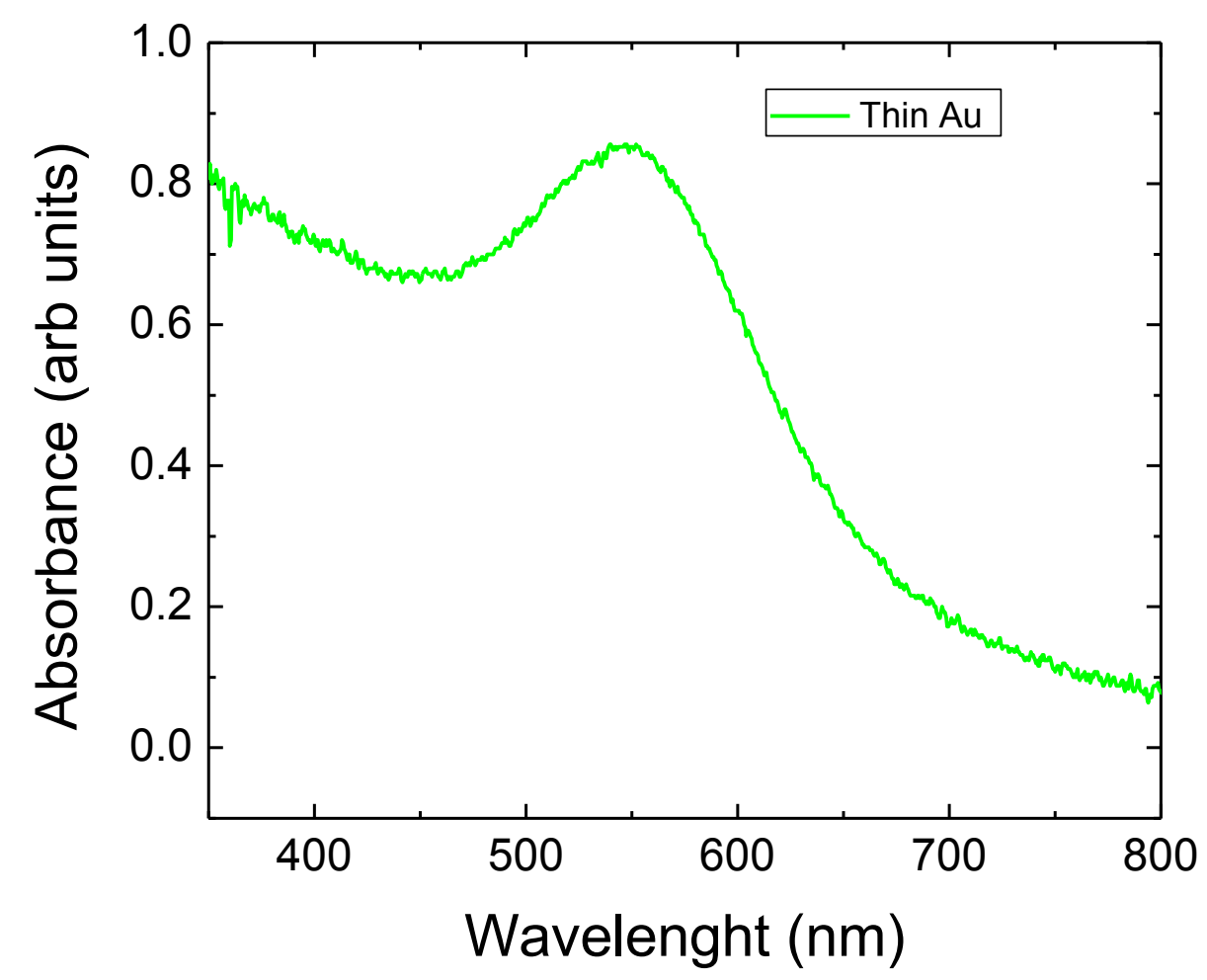

Figure 6.5. The absorbance of a very thin film of $\mathrm{Au}$. The absorbance signature of $\mathrm{Au}$ surface plasmons centered at $\sim 550 \mathrm{~nm}$ is clearly seen. 
The existence of an SERS effect at the Au-pentacene interface necessitates that there exist a nano-structured Au layer. In order to understand the nature of the nanostructured Au layer, we took scanning electron microscopy (SEM) images of the top surface of a pentacene specimen with a $2 \mathrm{~nm}$ layer of Au. As seen from Figure 6.2, the SERS effect just begins to show itself in specimens with Au thicknesses of $2 \mathrm{~nm}$, so we expected to see evidence of the nanostructure at the interface in the SEM surface image. Images were taken with a FEI Quanta 600F environmental SEM with an accelerating voltage of $20 \mathrm{kV}$. A typical image is shown in Figure 6.6. The images clearly show a surface roughness indicative of growth of Au islands. The typical diameter of the surface features is $180 \mathrm{~nm}$ $( \pm 60 \mathrm{~nm})$. Au nanostructures of this size will exhibit surface plasmons, consistent with the appearance of the SERS effect. 


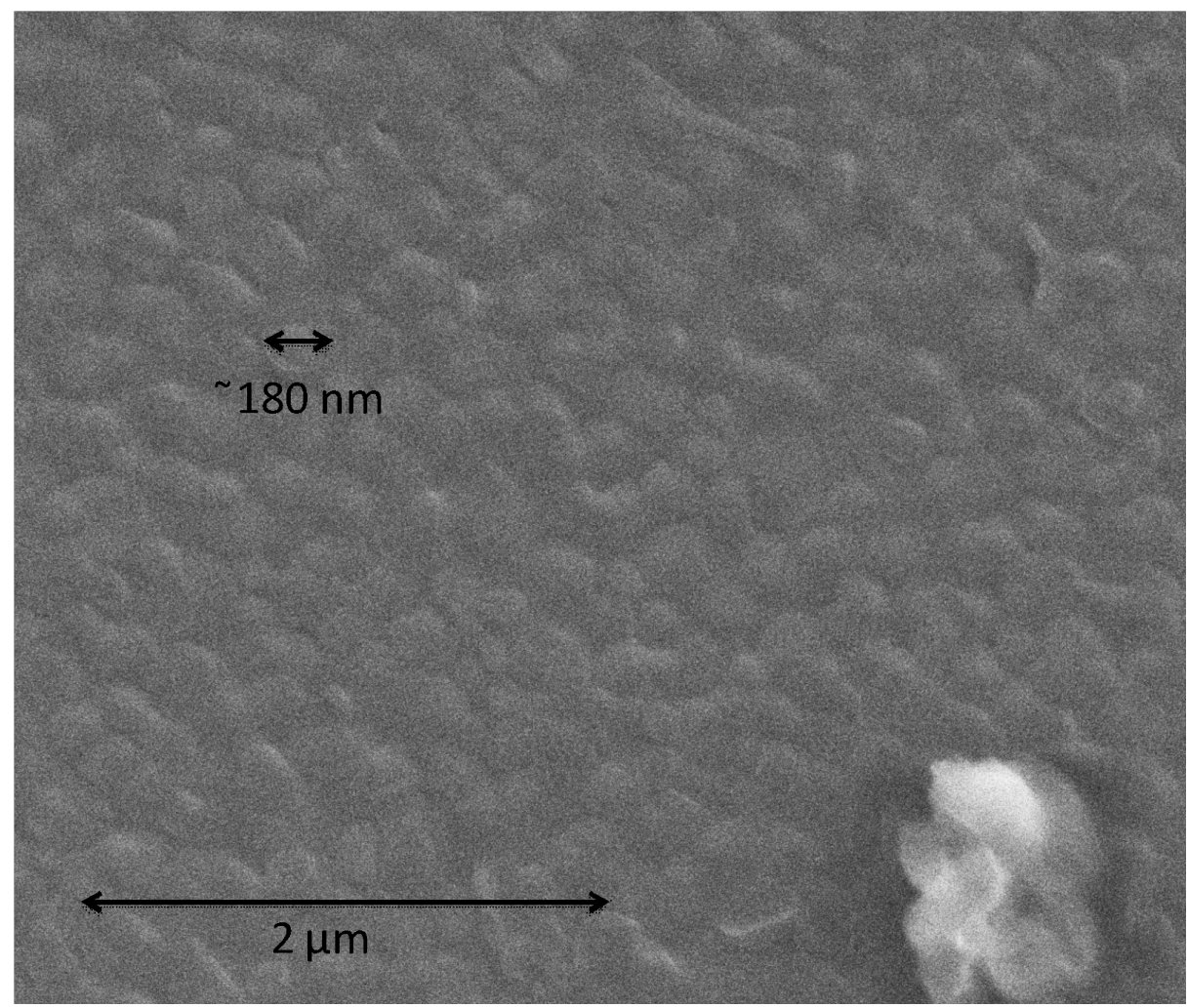

Figure 6.6. SEM image of the top surface of a $2 \mathrm{~nm}$ Au layer grown on top of a pentacene film. Nano-features consistent with the appearance of an SERS effect are clearly seen. An arrow is drawn at the upper left to highlight the characteristic size of the nano-features. The piece of dust in the lower right corner should be disregarded.

As shown in Figure 6.2, there are almost no changes in the Raman spectrum of pentacene when the Au layer thickness is less than $0.5 \mathrm{~nm}$. However, Figure 6.3 (b) and (c) suggest a permanent change due to a structural disorder of the pentacene molecule upon evaporation of thicker films of Au. A clue as to the origin of this permanent change can be found in the SERS spectrum of HOPG (Figure 6.4). Here, the enhancement picks up evidence of the D-band of disordered graphite. It is clear that the evaporation of $\mathrm{Au}$ onto the graphite creates some disorder in the graphite, which is then detectable via the 
SERS effect. This brings up the intriguing possibility that the SERS spectrum of pentacene contains a fingerprint of disorder in the pentacene film. If this is the case, then one may be able to correlate features in the SERS spectrum with molecular structure in the film.

\subsection{Theoretical Calculations of the Raman Spectrum of Pentacene}

As mentioned in section 2.4, DFT allows us to make theoretical predictions about the Raman frequencies and intensities. To understand the changes in the Raman spectrum of pentacene with $\mathrm{Au}$, we have calculated the Raman spectrum of pure and perturbed pentacene molecules by disrupting the aromaticity of the phenyl ring. As a first attempt to incorporate structural disorder in the pentacene molecule, an additional hydrogen atom was added to have one of the $\mathrm{C}$ atoms with $\mathrm{sp}^{3}$ hybridization. An addition of a hydrogen atom may not be the most realistic pathway but it allows us to introduce distortion in the phenyl rings to emulate the experimental conditions. The Raman spectrum was calculated for three different scenarios: unperturbed pentacene, pentacene with one $\mathrm{sp}^{3} \mathrm{C}$ in the outermost ring, and pentacene with one $\mathrm{sp}^{3} \mathrm{C}$ in the center ring (Figure 6.7 (a)). The three calculated spectra were weighted evenly and then averaged. 


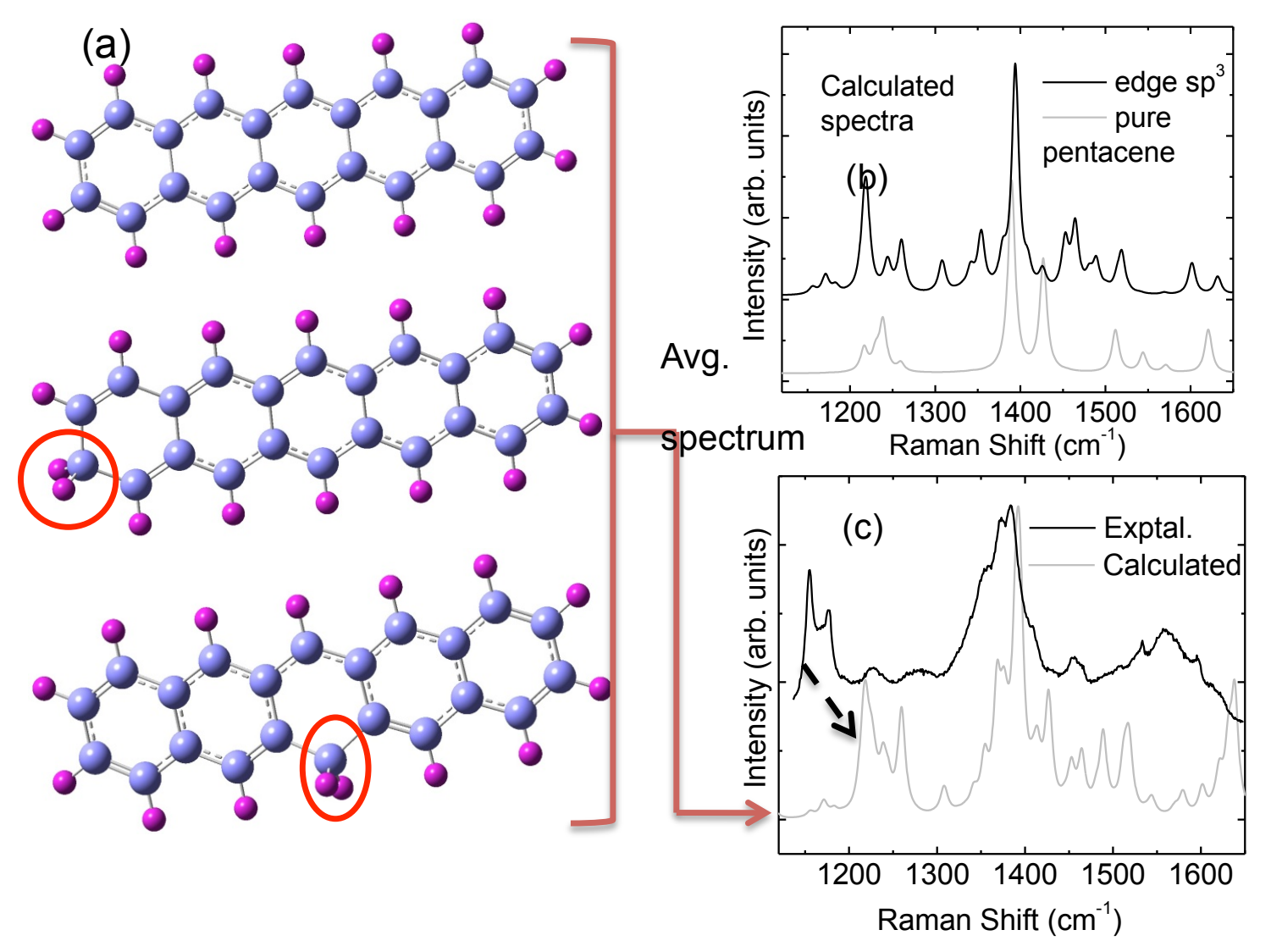

Figure 6.7. (a) Chemical structure of a pentacene molecule and pentacene with disordered $\mathrm{sp}^{2}$ rings. (b) Calculated Raman spectra of a pristine molecule and disordered molecule with an $\mathrm{sp}^{3} \mathrm{C}$ atom at the edge. (c) Experimental Raman spectrum of pentacene through a Au layer. The calculated spectrum is the average of the three spectra corresponding to the different schemes in (a). The dashed line correlates the experimental Raman peaks in the $1170 \mathrm{~cm}^{-1}$ region to the calculated spectrum.

Figure 6.7 (b) shows the calculated spectra from a pure pentacene molecule along with a pentacene molecule with a $\mathrm{sp}^{3}$ defect site in one of the edge phenyl rings. In the distorted molecule, the $1218 \mathrm{~cm}^{-1}$ peak gets enhanced and the aromatic C-C stretch 
vibration at $\sim 1390 \mathrm{~cm}^{-1}$ is slightly hardened compared to the pristine molecule. The calculated spectrum in Figure 6.7 (c) is an average of the three calculated spectra, as explained previously. This average spectrum agrees quite well with experiment - the energies are slightly different but the experimental $1170 \mathrm{~cm}^{-1}$ region is well reproduced by our calculations; similarly, the main features in the $1380 \mathrm{~cm}^{-1}$ region also corroborates with experiment.

By weighting the individual spectrum equally from the three pentacene molecules (in Figure 6.7), we do not suggest that the distorted molecules to the pristine molecule are in a 2/3 ratio. Clearly, upon Au deposition the pentacene layer is most likely to be affected at its interface with the Au. Here, where the pentacene is most disordered, the enhancement will be greatest. Therefore, by taking a $2 / 3$ ration of disorded to pristine molecules, we ensure this enhancement is reflected in our calculations. The experimental data (with the $785 \mathrm{~nm}$ laser) probes the bulk of the pentacene film. The SERS signal from the distorted molecules is much higher compared to the regular Raman intensity from pentacene. Using the $514 \mathrm{~nm}$ laser excitation source (which doesn't result in SERS), we clearly see that the pristine pentacene $\mathrm{C}-\mathrm{C}$ stretch at $1370 \mathrm{~cm}^{-1}$ is still stronger than the high-energy shoulder in Figure 6.3 (c). The increase in the $1560 \mathrm{~cm}^{-1}$ region (Figure 6.2) most likely corresponds to the G-band of $\mathrm{sp}^{2}$ carbons. ${ }^{65}$ This band appears in many disordered carbon structures - carbon fibers, glassy carbon blacks, and pregraphitic carbon. ${ }^{66}$ Overall, Au evaporation on the pentacene layer results in modifying the molecule and introducing some disorder in the $\mathrm{sp}^{2}$ carbon network. This may affect a small fraction of the pentacene molecules near the interface but since the SERS effect is strong for the disordered molecule, the experimental Raman spectrum is overwhelmed by 
the $1380 \mathrm{~cm}^{-1}$ and the $1560 \mathrm{~cm}^{-1}$ region. In other words, the SERS effect shows a sensitivity to the disorder that is otherwise undetectable.

While we can use $\mathrm{sp}^{3}$ carbons to simulate disorder in pentacene to reliably recreate the SERS spectrum, such molecular structures likely do not correspond the real structure of the pentacene in the film. Indeed, one may simply ask the question "what caused the carbon to become $\mathrm{sp}^{3}$ hybridized?" While the model provides for a quick way to simulate disorder and in the process provides critical insight, it does not give an adequate explanation of the molecular origins of the disorder.

A next attempt to simulate the SERS spectrum of pentacene was made by incorporating oxygen atoms into the structure. Pentacene, being in organic molecule dense with easily picked off $\pi$ electron, certainly can be oxidized. In fact, work has been done where the likely structures of oxidized pentacene were determined using mass spectroscopy. ${ }^{67}$ An attempt made to simulate the SERS spectrum using these structures is documented in appendix A. It is found that oxidized pentacene molecules cannot account for the observed the SERS spectrum. Thus, we look to another source for the origin of disorder in the pentacene molecule.

As the SERS effect requires the interaction of gold and pentacene as the metalsemiconductor interface, it is natural to turn to Au atoms when attempting to model the SERS spectrum. By explicitly considering the Au-pentacene interaction, we hope to find a more authentic model for the disorder in the pentacene film. In postulating how Au may interact with pentacene at the molecular level, we consider many possible configurations. Since the SERS effect may be caused both by direct bonding of the metal and the molecule or by a charge transfer effect, we consider geometries both where Au is bonded 
to pentacene molecules and where $\mathrm{Au}$ atoms are merely in close proximity to pentacene molecules. While a full simulation of the SERS effect would require incorporating the local field enhancement due to Au nanostructures at the interface, for computational simplicity we restrict ourselves to the interaction of individual $\mathrm{Au}$ atoms and pentacene molecules. Since one would expect that during the evaporation process, some Au atoms penetrate the top surface of the pentacene film and lie in between pentacene molecules, the geometries considered here are a reasonable simplification. By studying the interaction of individual $\mathrm{Au}$ atoms and pentacene molecules, we aim to understand the interface interaction at the most fundamental level.

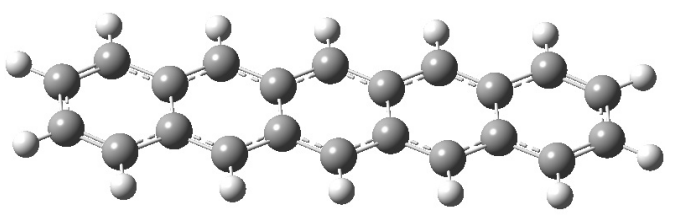

(a)

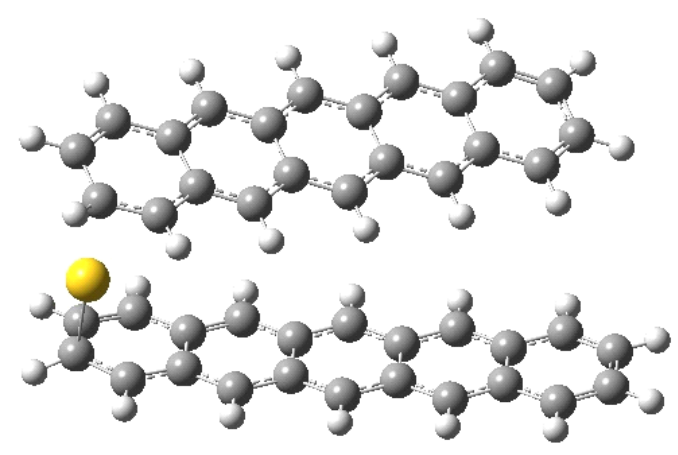

(b)

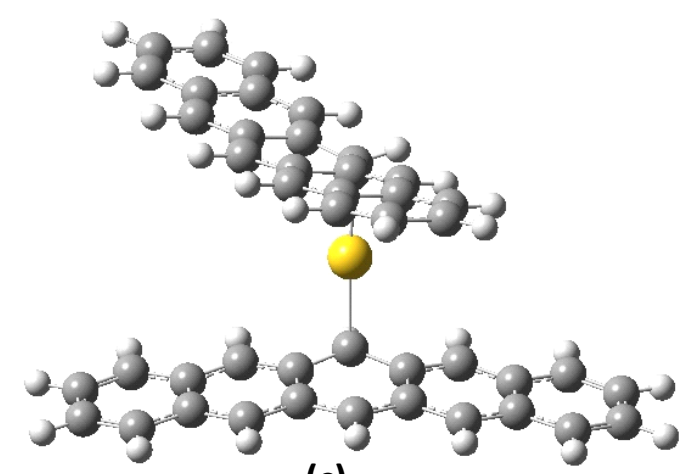

(c)

Figure 6.8. Optimized structures of (a) a pentacene molecule, (b) two pentacene molecules with a $\mathrm{Au}$ atom bonded to one of the pentacene molecules at the edge (Edge $\mathrm{Au})$, and (c) Au atom bonded to two pentacene molecules at the center (Center $\mathrm{Au})$. 
Figure 6.8 (a) shows an optimized geometry of a single pentacene molecule, and (b)(c) show two configurations of a $\mathrm{Au}$ atom and pentacene molecules. These are the minimal energy configurations obtained by DFT computations. In Figure 6.8 (b) the Au atom is $\sim 2 \AA$ away from each of the pentacene molecules over the outer rings (henceforth referred to as edge $\mathrm{Au}$ ). It is bonded to one of the molecules and not bonded to the other. In Figure $6.8(\mathrm{c})$, the Au atom is bonded to carbon atoms in the central rings and is positioned $\sim 2 \AA$ away from each pentacene molecule (henceforth referred to as center $\mathrm{Au})$. In both cases, the pentacene molecules are slightly deformed from their pristine configurations. Different geometries with the Au atom in various locations relative to the pentacene molecule were submitted for optimization, but all optimized to one of the above geometries. In both cases, the aromaticity of the pentacene molecule is disrupted by the presence of the $\mathrm{Au}$ atom. That is to say, the $\mathrm{Au}$ atom adds itself to a carbon atom resulting in a lone $\mathrm{sp}^{3}$ carbon bonded to both $\mathrm{Au}$ and hydrogen. We note that in neither case does the Au atom affect the breaking of bond in the pentacene molecule or the removal of a $\mathrm{H}$ atom. The break in the $\mathrm{sp}^{2}$ network of the pentacene molecule results in a change of the geometry of the molecule along with a change in its vibrational spectrum. 

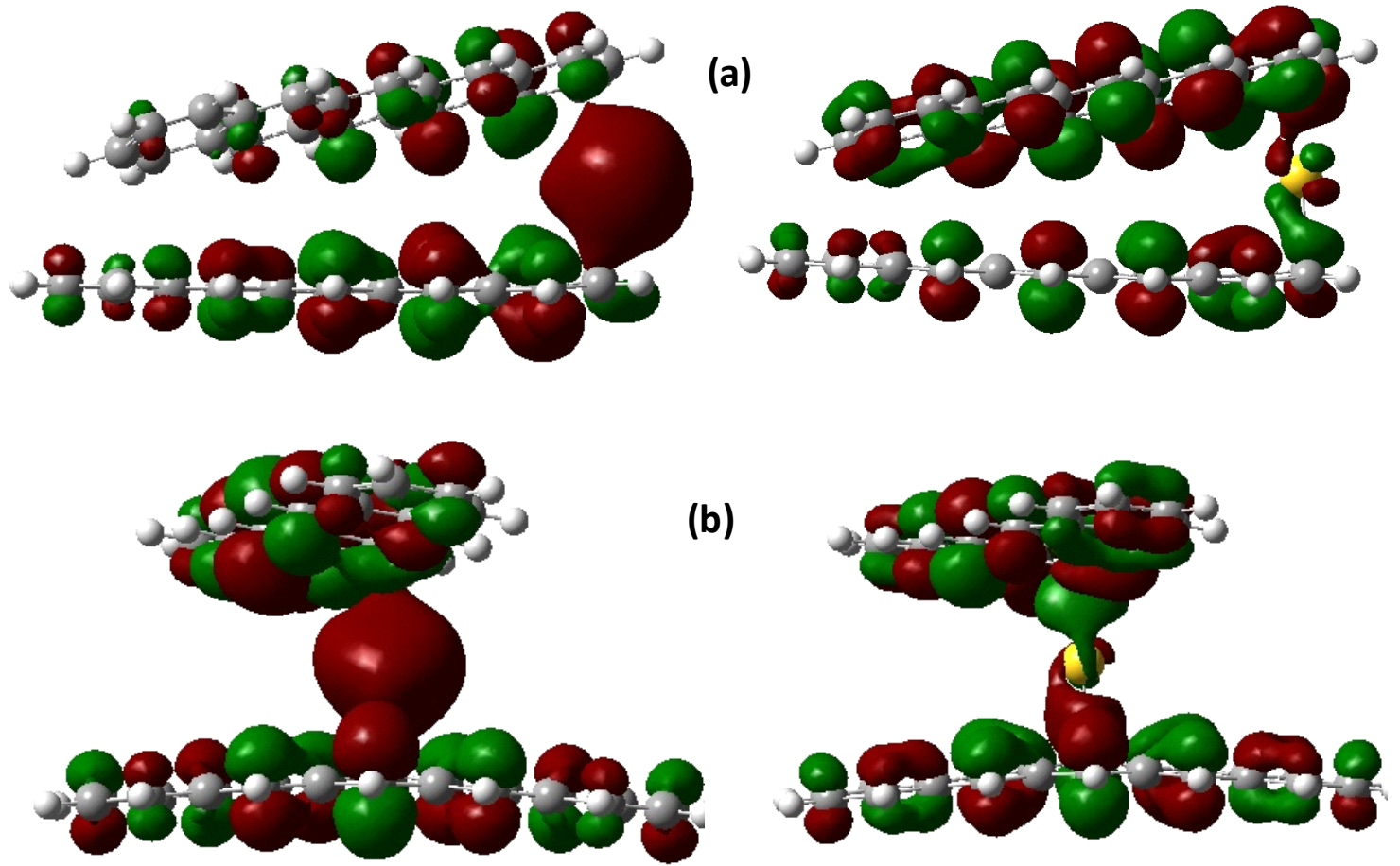

Figure 6.9. The HOMO (left) and LUMO (right) molecular orbitals of the Aupentacene systems for (a) the edge Au and (b) center Au conformation

The effect of the Au atom on the electronic structure of pentacene is clearly seen in Figure 6.9, where the HOMO (left) and LUMO (right) of the two above configurations are shown. The red and the green represent the phase of the molecular orbital. It is clear from the shape of the orbitals that there is some amount of charge transfer from the $\mathrm{Au}$ atom to the pentacene molecules, as one would expect from observing the SERS effect. The LUMO charge distribution for both Au pentacene configurations are roughly the same, which is also reflected in similar Raman scattering cross-sections. The Raman scattering cross-section is $\sim 10^{-28} \mathrm{~cm}^{2} / \mathrm{sr}$ for the Au-pentacene configurations, an order of magnitude higher than a pristine pentacene molecule. 
Since pentacene crystallizes in a herringbone pattern, ${ }^{68}$ one may ask if geometries where the two pentacene molecules are in a herringbone arrangement yield very different vibrational spectra. To answer this, we computed the electronic structure and vibrational energies of two pentacene molecules in the herringbone structure and found that the results were virtually identical to that of the single pentacene molecule. Details are provided in appendix B.This is expected since the Raman frequencies in our range of interest $\left(1000-1700 \mathrm{~cm}^{-1}\right)$ are all intra-molecular in nature - the only noticeable difference was a $\times 2$ enhancement of all the Raman peak intensities for the herringbone arrangement.

We further computed the electronic structure and vibrational energies of pentacene molecules in the herringbone structure bonded with $\mathrm{Au}$ atoms. We found, once again, that results were very similar to the results from the structures presented in Figure 6.8. The dominant intra-molecular vibrations of the Au-pentacene structure are dependent only on the nature of the Au-pentacene interaction and not on the relative orientation of the two pentacene molecules. Thus, for simplicity we use the three structures given in Figure 6.8 as representatives for the Au-pentacene interaction in crystalline pentacene films. The HOMO-LUMO energy gaps of the various Au pentacene conformations from our DFT calculations are given in Table 6.1. For comparison, the experimental HOMO-LUMO gap of pentacene is found to be in the range of $1.9 \mathrm{eV}$ to $2.2 \mathrm{eV},{ }^{69}$ where the HOMO energy is $\sim-4.9 \mathrm{eV}$ and the LUMO energy is $\sim-3.0 .^{70}$ Hybrid functionals within DFT with increasing fraction of Hartree-Fock exchange enhance the energy gap compared to local density approximation. ${ }^{71}$ We find the HOMO-LUMO energy gap of pentacene to be very close to the experimental value with the B3LYP functional. 
Table 6.1. Calculated HOMO-LUMO energy gap of pentacene and pentacene-Au conformations.

\begin{tabular}{|c|c|}
\hline Structure & HOMO-LUMO (eV) \\
\hline Pentacene & 2.29 \\
\hline Center Au & 2.11 \\
\hline Edge Au & 2.02 \\
\hline Pentacene (herringbone) & 2.00 \\
\hline $\begin{array}{c}\text { Pentacene herringbone with center } \\
\mathrm{Au}\end{array}$ & 1.84 \\
\hline Pentacene (experiment) $^{a}$ & 2.2 \\
\hline
\end{tabular}

Next, we attempt to correlate the theoretically expected changes of the vibrational spectrum of pentacene to our experimental results. The theoretically computed Raman spectra of pentacene and pentacene with $\mathrm{Au}$ are compared with the measured Raman spectra from pentacene films in both the channel and contact region of pentacene OFETs. As shown in section 6.1, when probed through the Au contact, we see a clear change in the pentacene spectrum consisting of shifts in peak position, selective enhancement of several peaks and the appearance of new peaks, all indicative of the SERS effect

As was mentioned earlier, the spectrum of oligoacenes in the $1000-1600 \mathrm{~cm}^{-1}$ region can roughly be split into two regions: the $\mathrm{C}-\mathrm{H}$ in-plane bending region at $1000-1300$ $\mathrm{cm}^{-1}$ and the C-C stretching region at $1300-1650 \mathrm{~cm}^{-1} \cdot{ }^{72}$ Since our discussion of the pentacene Raman spectrum will be limited to two clusters of peaks centered at $1180 \mathrm{~cm}^{-1}$ and $1380 \mathrm{~cm}^{-1}$ respectively, we will refer to the $1100-1250 \mathrm{~cm}^{-1}$ region as the $\mathrm{C}-\mathrm{H}$ bend region and the $1350-1450 \mathrm{~cm}^{-1}$ region as the $\mathrm{C}-\mathrm{C}$ stretch region, for convenience. The $\mathrm{C}-\mathrm{H}$ bend region of the spectrum is dominated by the presence of a peak at $1158 \mathrm{~cm}^{-1}$ corresponding to the $\mathrm{C}-\mathrm{H}$ bend of the atoms located at the end of the molecule and a peak at $1178 \mathrm{~cm}^{-1}$ corresponding to the $\mathrm{C}-\mathrm{H}$ bend of atoms located along sides of the 
molecule ${ }^{73}$ Here, we refer to these modes as outer ring $\mathrm{C}-\mathrm{H}$ bend and inner ring $\mathrm{C}-\mathrm{H}$ bend, respectively. The $\mathrm{C}-\mathrm{C}$ stretch region primarily consists of $\mathrm{C}-\mathrm{C}$ stretch vibrations localized on either the center or outer rings of molecule. These modes are referred to as either inner or outer ring breathing modes, respectively.

In order to calculate the SERS spectrum of pentacene films in the Au contact region of the OFETs, we incorporate the Au-pentacene interaction using the structures discussed in Figure 6.7. Figure 6.10 shows the Raman spectra of the two pentacene molecules with $\mathrm{Au}$ incorporated as discussed earlier. In both cases, the spectra are dramatically changed compared to the pristine molecule. The changes arise mainly due to the four following features: (1) silent Raman peaks for pristine pentacene become Raman active; (2) the intensity of some peaks are greatly enhanced relative to that of pristine pentacene; (3) energy positions of some pristine pentacene peaks are shifted; (4) most pristine pentacene peaks are split into new vibrations of unequal intensity. For (4), the splitting usually results in a doublet of vibrations where each of the peaks is localized on one of the two molecules. The location of the $\mathrm{Au}$ atom along with the nature of its interaction with pentacene (direct bond or proximity interaction) has a dramatic effect on which of the four above features the Raman spectrum most heavily depends upon. 


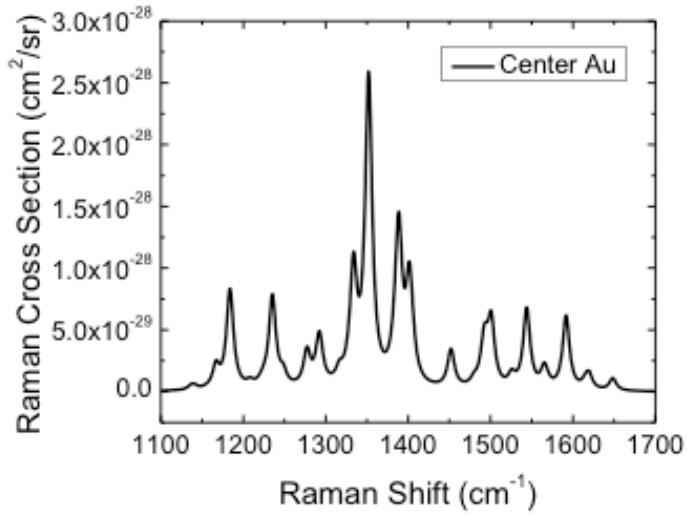

(a)

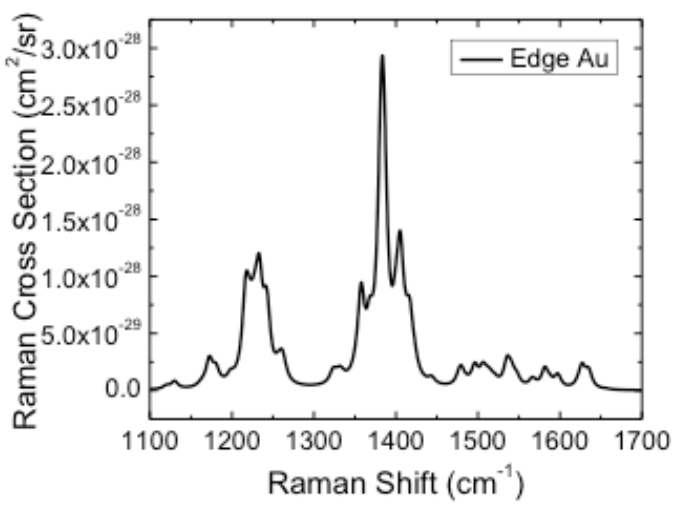

(b)

Figure 6.10. Calculated Raman spectrum of pentacene molecules with (a) center $\mathrm{Au}$ and (b) edge Au.

Table 6.2 indicates the position and nature of the vibrations in pristine pentacene and each of the two Au-disordered configurations for the calculated Raman spectra. Since we compare the frequencies of pristine pentacene to the Au-pentacene complexes, we have denoted the symmetries of the vibrations to understand the origin of the vibrations. We note that the mode symmetries correspond only to the pristine case. In the edge Au case, the two main peaks seen in the $\mathrm{C}-\mathrm{H}$ bend region of the spectrum have been broadened into a band of many peaks. The formerly dominant $\mathrm{a}_{\mathrm{g}} \mathrm{C}-\mathrm{H}$ bend at $1238 \mathrm{~cm}^{-1}$ (mode 4) is overshadowed by the strongly enhanced $b_{3 g}$ (mode 3 ) peaks, which are seen at slightly lower energies. The $\mathrm{a}_{\mathrm{g}} \mathrm{C}-\mathrm{H}$ bend at $1216 \mathrm{~cm}^{-1}$ (mode 2) has been weakened in its former location and appears as three peaks at slightly higher energies. We note that when peaks are split into doublets, the vibration of higher energy is consistently localized on the nonbonded pentacene. In the center $\mathrm{Au}$ case, the $\mathrm{C}-\mathrm{H}$ bend region is dominated by the $\mathrm{b}_{3 \mathrm{~g}}$ mode (mode 3 ) doublet at 1235 and $1236 \mathrm{~cm}^{-1}$. The vibration has been slightly shifted to 
higher energies compared to the pristine case and greatly enhanced. The two $\mathrm{a}_{\mathrm{g}}$ modes (modes 2 and 4) in this region are weakened significantly and hardly contribute. The only other significant contributor to the spectrum in this region is the appearance of the $b_{2 u} C$ $\mathrm{H}$ bend mode doublet at 1183 and $1184 \mathrm{~cm}^{-1}$. This mode was previously silent.

In the $\mathrm{C}-\mathrm{C}$ stretch region, the edge Au configuration is marked by a strong central peak centered at $1380 \mathrm{~cm}^{-1}$ with shoulders at $1360 \mathrm{~cm}^{-1}$ and $1405 \mathrm{~cm}^{-1}$. The center peak is primarily from an enhancement and splitting of the $a_{g}$ breathing mode originally seen at $1389 \mathrm{~cm}^{-1}$ (mode 8 ). The high energy shoulder is primarily due to $b_{2 u}$ breathing modes originally seen at 1400 and $1429 \mathrm{~cm}^{-1}$ (modes 9 and 11), and the low energy shoulder is due to the $b_{2 u}$ mode originally seen at $1367 \mathrm{~cm}^{-1}$ (mode 7). Both of these sets were silent in the pristine case. For the center Au case, the spectrum is dominated by the $\mathrm{b}_{3 \mathrm{~g}}$ mode (mode 6) doublet at 1352 and $1353 \mathrm{~cm}^{-1}$. This previously silent mode appears at the same energy as in the pristine system. The previously strong $a_{\mathrm{g}}$ ring breathing mode (mode 8 ) has been weakened considerably and does not contribute significantly anymore. A $b_{2 u} C$ C stretch mode (mode 7) doublet at 1388 and $1389 \mathrm{~cm}^{-1}$ provides the second strong peak in the region. This mode was also previously silent. The third peak in the region is an $\mathrm{a}_{\mathrm{g}}$ ring breathing mode (mode 10), which has been slightly enhanced, shifted to lower energies, and split. One immediately sees how the unmistakable appearance of previously silent peaks and the shifting of other peaks may begin to explain the SERS spectrum. 
Table 6.2: Calculated Raman frequencies and their strengths of pristine pentacene and pentacene molecules with an edge $\mathrm{Au}$ atom and a center $\mathrm{Au}$ atom. The symmetries correspond only to pristine pentacene. The peak positions are in $\mathrm{cm}^{-1}$.

\begin{tabular}{|c|c|c|c|c|c|c|c|}
\hline \multirow{3}{*}{ Mode } & \multirow[b]{2}{*}{ Description } & \multicolumn{2}{|l|}{ Pristine } & \multicolumn{2}{|l|}{ Edge Au } & \multicolumn{2}{|c|}{ Center Au } \\
\hline & & Position & Intensity & Position & Intensity & Position & $\begin{array}{l}\text { Intensity } \\
\end{array}$ \\
\hline & C-H Bend Region & & & & & & \\
\hline \multirow[t]{2}{*}{1} & $\mathrm{~b}_{2 \mathrm{u}}($ Edge $\mathrm{C}-\mathrm{H}$ bend $)$ & 1155 & silent & 1162 & v. weak & 1183 & med \\
\hline & & & & 1165 & v. weak & 1184 & med \\
\hline \multirow[t]{4}{*}{2} & $a_{g}$ (Outer ring $\mathrm{C}-\mathrm{H}$ & 1216 & weak & 1200 & v. weak & 1219 & v. weak \\
\hline & bend) & & & 1220 & med & 1220 & v. weak \\
\hline & & & & 1221 & weak & 1230 & v. weak \\
\hline & & & & & & 1231 & v. weak \\
\hline \multirow[t]{4}{*}{3} & $\mathrm{~b}_{3 \mathrm{~g}}$ (Inner ring twist) & 1230 & weak & 1216 & strong & 1235 & med \\
\hline & & & & 1224 & med & 1236 & med \\
\hline & & & & 1228 & med & & \\
\hline & & & & 1233 & strong & & \\
\hline \multirow[t]{2}{*}{4} & $\mathrm{a}_{\mathrm{g}}$ (Inner ring $\mathrm{C}-\mathrm{H}$ & 1238 & med & 1241 & med & 1208 & weak \\
\hline & bend) & & & 1243 & med & & \\
\hline \multirow[t]{3}{*}{5} & $\mathrm{~b}_{2 \mathrm{u}}$ (Anti-symmetric & 1265 & silent & 1261 & med & 1277 & med \\
\hline & inner ring $\mathrm{C}-\mathrm{H}$ bend) & & & 1263 & weak & 1278 & med \\
\hline & C-C Stretch Region & & & & & & \\
\hline \multirow[t]{2}{*}{6} & $b_{3 g}$ & 1353 & v. weak & 1351 & weak & 1352 & v. strong \\
\hline & & & & & & 1353 & v. strong \\
\hline \multirow[t]{2}{*}{7} & $b_{2 u}$ & 1367 & silent & 1358 & strong & 1388 & strong \\
\hline & & & & 1368 & med & 1389 & strong \\
\hline \multirow[t]{3}{*}{8} & $\mathrm{a}_{\mathrm{g}} \quad$ (Inner & 1389 & strong & 1381 & strong & 1380 & v. weak \\
\hline & breathing) & & & 1382 & v. strong & 1384 & med \\
\hline & & & & 1383 & v. strong & & \\
\hline \multirow[t]{2}{*}{9} & $\mathrm{~b}_{2 \mathrm{u}} \quad$ (anti-symmetric & 1400 & silent & 1400 & med & - & - \\
\hline & inner ring breathing) & & & 1405 & v. strong & & \\
\hline \multirow[t]{2}{*}{10} & $\mathrm{a}_{\mathrm{g}}$. (outer & 1427 & med & 1411 & med & 1401 & med \\
\hline & breathing) & & & & & 1402 & strong \\
\hline \multirow[t]{2}{*}{11} & $\mathrm{~b}_{2 \mathrm{u}} \quad$ (anti-symmetric & 1429 & silent & 1416 & med & 1405 & weak \\
\hline & outer ring breathing) & & & 1417 & med & 1405 & weak \\
\hline
\end{tabular}

One does not expect either of the two Au-pentacene configurations to alone account for the features seen in the experimental SERS spectra. In fact, the experimental spectrum should be the sum of spectra from various types of Au-induced disordered pentacene molecules along with pristine molecules spread throughout the sampling volume. The computed spectra from structures given here, however, are representative of the features one would observe in experimental SERS. To simulate this effect, we average together 
the two Au-disordered spectra with the spectrum from pristine pentacene (weighted by a factor of 10) to get a spectrum which accounts for all the various shifts, enhancements, and splits that Au may induce into the Raman signature of pentacene. As before, the averaging is just a convenient form of combining the spectra and we do not mean to imply that $1 / 3$ of the sampling volume consists of one of structures given above. In fact, the vast majority of the molecules sampled will be unaffected by the Au atoms. However, we see from the vibrational results that the total intensity of the Raman peaks in Audisordered structures is much higher than that of the pristine pentacene. We aim to combine the spectra in such a way that features from both the pristine pentacene spectrum and the Au-disordered spectra contribute to the final spectrum. This is accomplished by weighting the pristine pentacene spectrum by a factor of 10 .

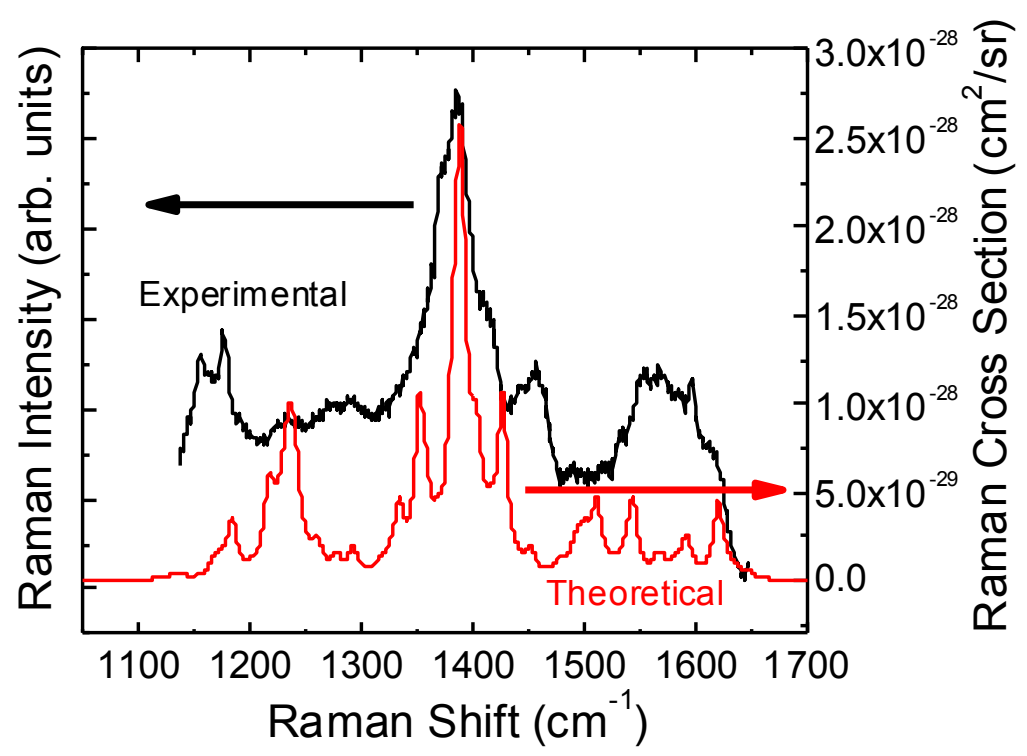

Figure 6.11. Experimental SERS spectrum of pentacene from a Au/pentacene/organic dielectric/Al OFET structure (probed through the Au contact). The calculated Raman spectrum is shown in red by averaging the two Au-pentacene configurations and the pristine pentacene, which is weighted as explained in the text. 
Figure 6.11 shows the average computed spectrum and the experimental SERS spectrum. The significant changes in the experimental data when measured through the $\mathrm{Au}$ contact is the enhancement in the $1380 \mathrm{~cm}^{-1}$ and $1560 \mathrm{~cm}^{-1}$ region (Fig. 3), corresponding to the D- and G- band of disordered $\mathrm{sp}^{2}$ carbons, respectively. ${ }^{74}$ The experimental data in Figure 6.11 is from a pentacene film on a organic dielectric based OFET structure, where the Au thickness was $\sim 30 \mathrm{~nm}$. It is expected that for thinner $\mathrm{Au}$ layers, the $1158 \mathrm{~cm}^{-1}$ peak would be more intense that the $1178 \mathrm{~cm}^{-1}$ peak. The calculated spectrum effectively recreates the features seen in the experimental data. Most prominently, the broad band in the $1380 \mathrm{~cm}^{-1}$ region is observed in the calculated spectrum. The broad features in the $1180 \mathrm{~cm}^{-1}$ and $1560 \mathrm{~cm}^{-1}$ region are also reproduced when consideration for expected discrepancies in theoretical and experimental energies is given. We note that the $\mathrm{C}-\mathrm{H}$ bend modes in the $1180 \mathrm{~cm}^{-1}$ region are strongly affected in the crystalline environment; the gas phase theoretical calculations are expected to show higher energies. It is clearly seen that disorder induced in pentacene molecules by $\mathrm{Au}$ atoms penetrating into the film can explain the unique surface-enhanced spectra seen at Au-pentacene interfaces. Although these calculations do not explicitly consider an electromagnetic enhancement of the local fields due to the SERS effect, the DFT calculations of the Raman spectrum of pentacene in the presence of Au nevertheless predict an enhanced Raman cross section. The edge Au and center Au structures show an order of magnitude higher Raman scattering cross section compared to the pristine molecule. 


\subsection{Pentacene Raman maps}

Since it is now apparent that the SERS effect exhibits a high sensitivity to disorder in the pentacene film, it reasonable to inquire if the effect can be harnessed as a diagnostic tool to study degradation and structural changes in organic film of OFETs as they are biased. It is known that OFET operation for many different polymers and molecules deteriorate upon bias stress, as usually observed as a lowering of the current, decreased mobility, and enhanced threshold voltages. ${ }^{75}$ This effect is especially pronounced in OFETs fabricated using inorganic dielectrics. On the other hand, pentacene OFETs fabricated using an organic low-k ploy(methyl methacrylate) (PMMA) gate dielectric have been shown to feature highly stable, low-voltage operation. ${ }^{76}$ These OFETs operated at very low voltages with threshold voltages $\sim-1 \mathrm{~V}$ and showed negligible hysteresis under gate bias stress. Others have used x-ray diffraction and Raman scattering to report on the origins of the improved performance of PMMA based devices. ${ }^{77}$ Other low- $k$ dielectrics such as poly (4-vinyl phenol) (PVP) based pentacene OFETs also show highly stable behavior with enhanced charge carrier mobility. As we now have two classes of pentacene devices, the $\mathrm{SiO}_{2}$ dielectric based, high-operating voltage devices and the organic dielectric based low-operating voltage devices, we can now compare the evolution of their SERS spectra as a function of bias stress to see if, indeed, there is evidence of greater degradation in the high-operating voltage devices.

To do this, we fabricated regular pentacene OFETs based both on $\mathrm{SiO}_{2}$ and PVP dieletric layers. $\mathrm{SiO}_{2}$ based devices were fabricated on untreated $\mathrm{SiO}_{2}$ surfaces using methods described in chapter 3. The Au source/drain pads of the devices shown here 
were $\sim 5 \mathrm{~nm}$ thick. Thin injecting electrodes were chosen so as to maximize the SERS sensitivity. As these OFETs were not very well optimized, the typical charge carrier mobility $(\mu)$ was in the mid $10^{-3} \mathrm{~cm}^{2} /$ Vs range. PVP based OFETs were fabricated using a similar top-contact bottom gate geometry. $8 \mathrm{wt} \%$ PVP $(\mathrm{Mw}=25,000 \mathrm{~g} / \mathrm{mol})$, purchased from Sigma-Aldrich, was dissolved in dimethyl sulfoxide (DMSO) and spincoated at $5000 \mathrm{rpm}$ on Al-coated glass. The films were then baked at $120^{\circ} \mathrm{C}$ for $1 \mathrm{~h}$ in order to achieve solvent evaporation. As with the $\mathrm{SiO}_{2}$ based devices, a $60 \mathrm{~nm}$ thick pentacene (purchased from Tokyo Chemical Industrial Co. Ltd and used without any purification) layer was thermally evaporated onto the dielectric surface at a rate of $0.2 \AA / \mathrm{s}$ at room temperature. Au top contacts of a full 30nm were evaporated to complete theses devices. Full thickness contacts were chosen for the organic dielectric devices in order to maximize device performance as opposed to maximizing SERS signal strength. The mobility of these PVP based devices were typically in the $\sim 10^{0} \mathrm{~cm}^{2} / \mathrm{Vs}$ range.

It should be emphasized that while both sets of devices (PVP and $\mathrm{SiO}_{2}$ based pentacene OFETs) were fabricated and characterized in identical environments, the vertical electric field in the low -operating voltage devices is an order of magnitude lower than that of the high-operating voltage devices. The typical operating voltage of $-3 \mathrm{~V}$ for the low-operating voltage devices corresponds to an electric field of $\sim 10^{5} \mathrm{~V} / \mathrm{cm}$. On the other hand, the operating voltage of $-40 \mathrm{~V}$ for the high-operating voltage devices corresponds to an electric field of $\sim 10^{6} \mathrm{~V} / \mathrm{cm}$. This suggests that any differences in the responses of the SERS spectra to bias stress are related to the relative field strengths.

Transfer characteristics of the pristine devices were measured immediately after fabrication. In addition, two-dimensional Raman maps of the OFETs were generated by 
measuring several individual Raman spectra in the channel region of the OFET and through the Au contact immediately adjacent to the channel region at $5 \mu \mathrm{m}$ intervals. Together, this set of measurement comprised the "pre-bias stress" results. The devices then underwent a bias stress by running them in the saturation regime $\left(\mathrm{V}_{\mathrm{G}}=-40 \mathrm{~V}\right.$ and $V_{\mathrm{D} \mathrm{S}}=-30 \mathrm{~V}$ for the $\mathrm{SiO}_{2}$ based devices and $\mathrm{V}_{\mathrm{G}}=\mathrm{V}_{\mathrm{DS}}=-3 \mathrm{~V}$ for the PVP based devices) for 20 minutes in air. Subsequently, the transfer characteristics and Raman maps of the OFETs were re-measured. This second set measurement comprised the "Post-bais stress" results. Comparing the pre-bias stress measurement with the post-bias stress measurements gave a clear picture of the evolution of the electrical and optical characteristics of the pentacene film as a function of electric field exposure.

Figure 6.12 shows the transfer characteristics of a $\mathrm{SiO}_{2}$ pentacene OFET both before and after being put under the bias stress. Here, the square root of the drain-source current is plotted to better visualize the change in threshold voltage. A 40\% drop in drain-source current was observed over the course of the bias stress. 


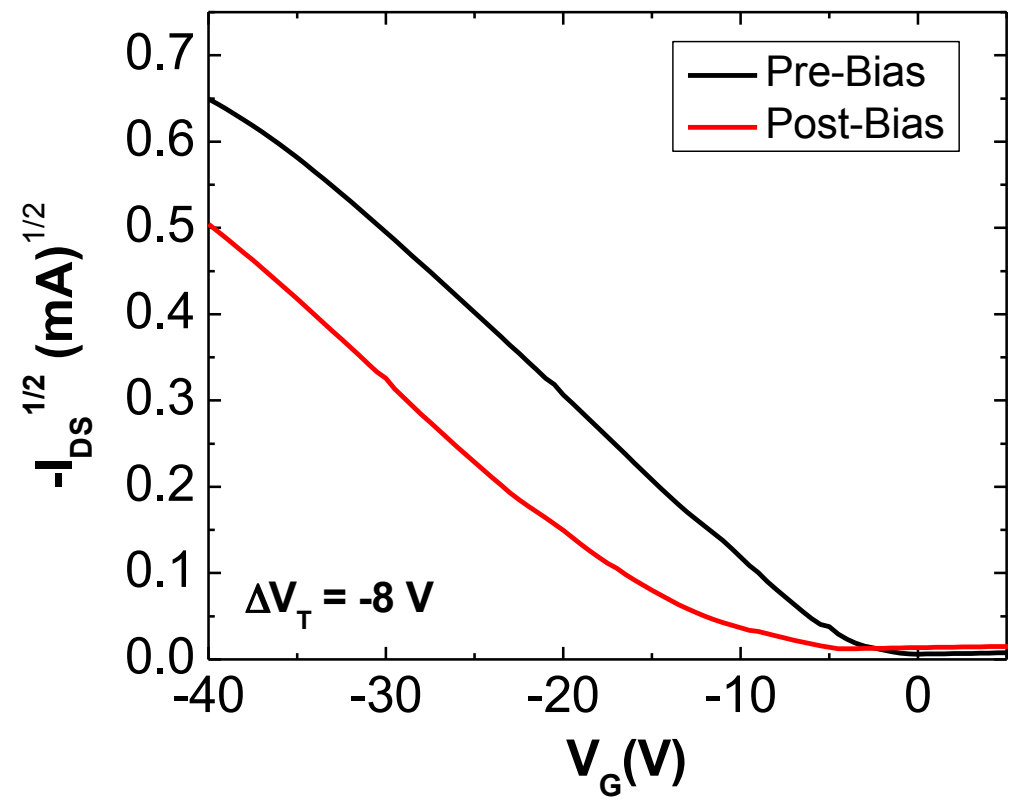

Figure 6.12. The transfer plots of a pentacene OFET before and after biasing for 20 minutes at $\mathrm{V}_{\mathrm{DS}}=-30 \mathrm{~V}$ and $\mathrm{V}_{\mathrm{G}}=-40 \mathrm{~V}$.

Device parameters such as charge carrier mobility, threshold voltage, and On/Off ratio were estimated by using the standard current-voltage characteristics of OFETs given by eq. 2.7. After biasing the device, the charge carrier mobility was found to decrease from $2.0 \times 10^{-3} \mathrm{~cm}^{2} / \mathrm{Vs}$ to $1.8 \times 10^{-3} \mathrm{~cm}^{2} / \mathrm{Vs}$. The threshold voltage increased from $-4 \mathrm{~V}$ to $-12 \mathrm{~V}$ and the On/Off ratio decreased from $\sim 10^{4}$ to $\sim 10^{3}$. It is evident that while the mobility remained relatively stable, the threshold voltage and On/Off ratio degraded considerably. Table 6.3 shows all device parameters.

Next, the transfer characteristics of a PVP based pentacene OFET were measured. Figure 6.13 shows the data for both before and after bias stress. As before, the common 
device performance parameters were estimated using the standard current-voltage characteristics. Charge carrier mobility was found to increase from $1.4 \mathrm{~cm}^{2} / \mathrm{Vs}$ to 1.8 $\mathrm{cm}^{2} / \mathrm{Vs}$. The threshold voltage remained almost unchanged, going from $-1.7 \mathrm{~V}$ to $-1.5 \mathrm{~V}$. Lastly, the On/Off ratio decreased from $\sim 10^{4}$ to $\sim 10^{3}$, similar to the previous device. The parameters are tabulated in table 4.1 . While the changes in the charge carrier mobility and On/Off ratio were similar in the two devices, the behavior of the threshold voltage was remarkably different. As expected, the $\mathrm{SiO}_{2}$ based device exhibited a strong degradation in threshold voltage while the PVP based device maintained a stable threshold voltage.

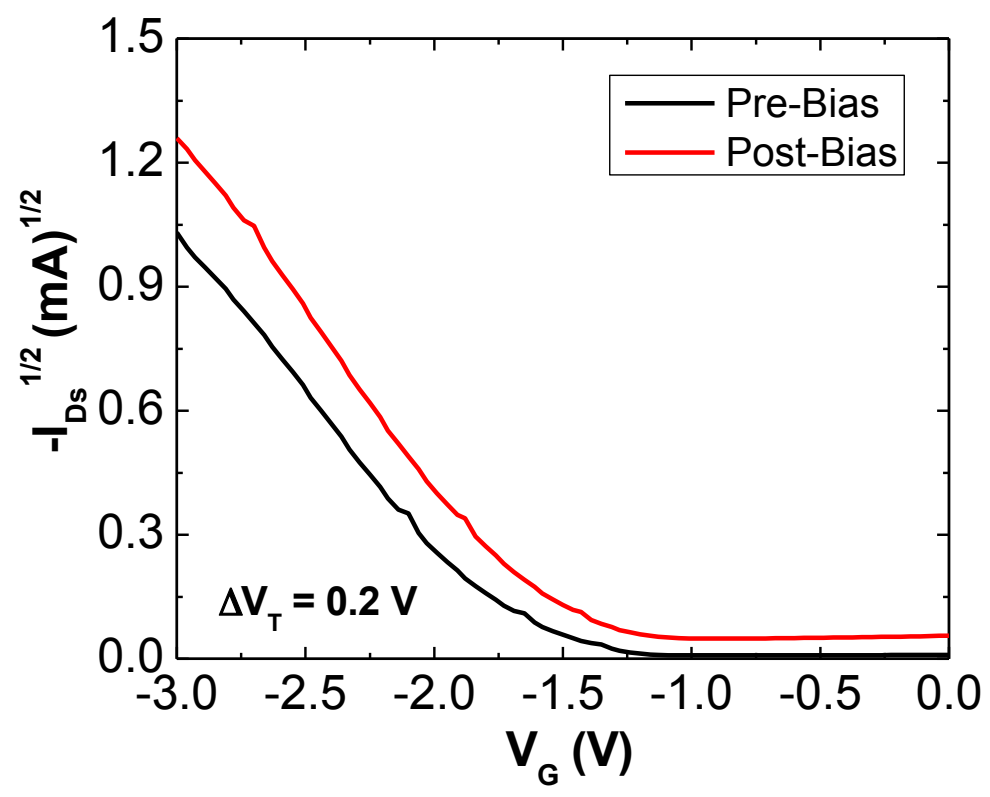

Figure 6.13. The transfer plots of a PVP based pentacene OFET before and after biasing for 20 minutes at $\mathrm{V}_{\mathrm{DS}}=-3 \mathrm{~V}$ and $\mathrm{V}_{\mathrm{G}}=-3 \mathrm{~V}$ 
Table 6.3. Device parameters for pentacene OFET before and after bias stress.

\begin{tabular}{|c|c|c|c|}
\hline & $\begin{array}{l}\text { Mobility } \\
\left(\mathrm{cm}^{2} / \mathrm{Vs}\right)\end{array}$ & $\begin{array}{l}\text { Threshold } \\
\text { Voltage } \\
\text { (V) }\end{array}$ & On/Off ratio \\
\hline \multicolumn{4}{|l|}{$\mathrm{SiO}_{2}$ based device } \\
\hline $\begin{array}{l}\text { Before bias } \\
\text { stress }\end{array}$ & $2.0 \times 10^{-3}$ & -4 & $\sim 10^{4}$ \\
\hline After bias stress & $1.8 \times 10^{-4}$ & -11 & $\sim 10^{3}$ \\
\hline \multicolumn{4}{|l|}{ PVP based device } \\
\hline $\begin{array}{l}\text { Before } \\
\text { stress }\end{array}$ & 1.4 & -1.7 & $\sim 10^{4}$ \\
\hline After bias stress & 1.8 & -1.5 & $\sim 10^{3}$ \\
\hline
\end{tabular}

As we had clearly confirmed difference in performance degradation between the highoperating voltage and low-operating voltage devices, we now attempted to correlate the degradation in OFET performance to changes in the Raman spectrum measured from the device. Each Raman linescan map consists of $\sim 13$ spectra spanning $60 \mu \mathrm{m}$; the first measurement $(0 \mu \mathrm{m})$ is taken in the channel itself, directly on pentacene, and the last measurement was on the Au pad. As shown in the optical image (Figure 6.1), the Raman spectra from the different points were collected to plot an intensity map, which provides an immediate snapshot as to how the spectrum changes across the interface. After bias stress, the Raman linescan maps were re-measured. Figure 6.14 shows the Raman maps from a $\mathrm{SiO}_{2}$ based pentacene OFET before and after bias stress using the $785 \mathrm{~nm}$ excitation source. The incident laser power and integration times were the same for both measurements. Data set 1 refers to the spectrum measured on the pentacene (see Figure 6.1) and data set 13 refers to the Raman spectrum measured well within the Au pad. All the spectra were measured in the $1100-1700 \mathrm{~cm}^{-1}$ region. It is immediately obvious that the spectrum changes as one moves from the channel region to the contact region (the contact begins at around dataset 6). This is due to the appearance of the SERS effect as 
discussed previously. A careful analysis of the Raman peaks show that there is a significant enhancement of the peak intensity $\left(1380 \mathrm{~cm}^{-1}\right.$ and $\left.1560 \mathrm{~cm}^{-1}\right)$ by noting the area under the curve, as well as an increase in line broadening.

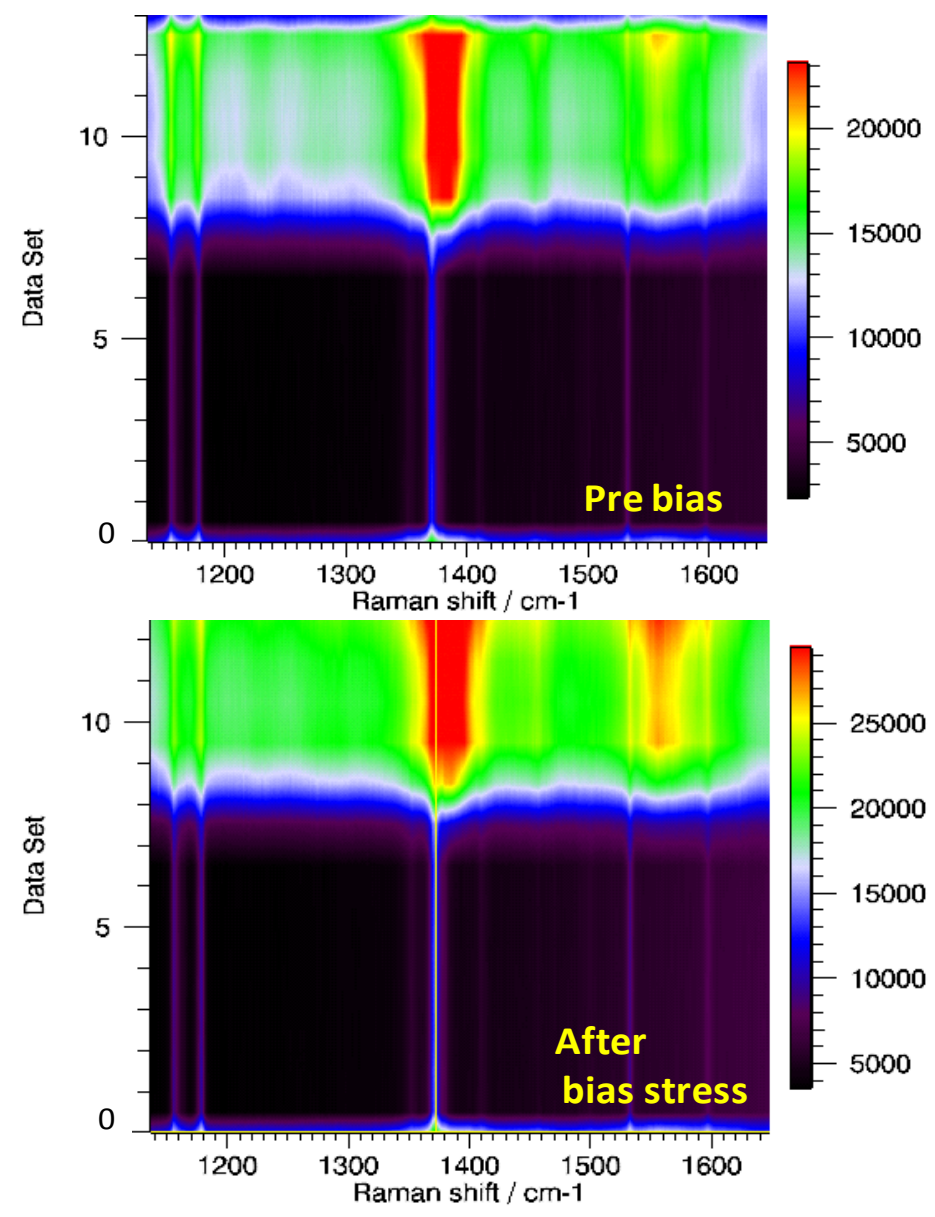

Figure 6.14. Raman linescan maps of a $\mathrm{SiO}_{2}$ pentacene OFET before after bias stress.

Data set 1 corresponds to the Raman spectrum measured in the channel region and data set 13 corresponds to the Raman spectrum well within the Au pad of one of the contacts

Our theoretical Raman spectrum calculations of pentacene indicate that an enhancement of the $1380 \mathrm{~cm}^{-1}$ region is a signature of the distortion of the molecule. By biasing the OFETs at $-40 \mathrm{~V}$, we subject them to an electric field of $\sim 10^{6} \mathrm{~V} / \mathrm{cm}$. The force 
due this field may be adequate in distorting the molecules with an increasing fraction of disordered $\mathrm{sp}^{2}$ carbons. Another scenario could relate to the actual transport through the OFET channel, which results in structural modifications to the molecule. Regardless, there is a permanent structural change in the pentacene caused by bias stress. Repeating the Raman measurement after several days yields similar Raman maps as shown in the "After bias stress" panel of Figure 6.14.

Next, we create similar Raman maps for the PVP based pentacene device. The maps are shown in Figure 6.15. Like before, the spectrum was recorded in the 1100 to 1700 $\mathrm{cm}^{-1}$ region. The laser intensities and integration times were identical for both measurements. It is apparent that the two maps are almost identical. That is to say, the Raman spectra of the pentacene film do not systematically change as a function of bias stress for the low-operating voltage OFETs, unlike high-operating $\mathrm{SiO}_{2}$ based pentacene OFETs. The slight differences in the two images arise since the maps were not measured from the exact same location. Many pairs of before/after maps were measured from several devices on different substrates and all were similar to what is reported here, assuring that the observed stability in the Raman spectra of the low-operating voltage devices is not an artifact of any one device or substrate. 


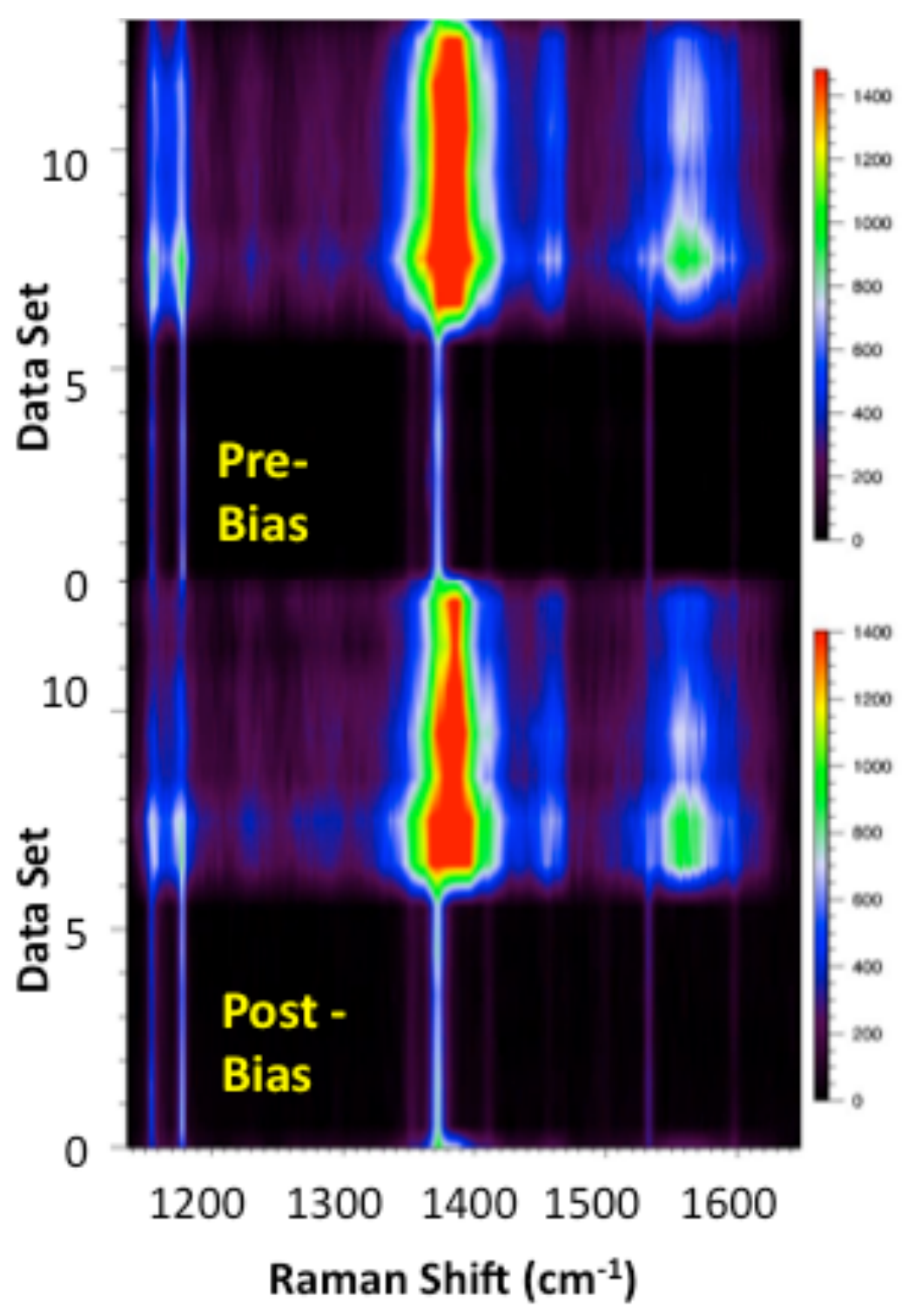

Figure 6.15. Raman linescan maps of a PVP based pentacene OFET before after bias stress. Data set 1 corresponds to the Raman spectrum measured in the channel region and data set 13 corresponds to the Raman spectrum well within the $\mathrm{Au}$ pad of one of the contacts 
To summarize this portion of the work, we demonstrate that $\mathrm{Au}$ evaporation on pentacene films induces disorder, which is enhanced by SERS. A comparison of our experimental results with theoretical DFT Raman spectrum calculations suggests the presence of disordered carbons in pentacene. This is confirmed by further DFT studies where the experimental SERS spectrum can be recreated by explicitly modeling the Aupentacene interaction at the metal-semiconductor interface. Au atoms enhance the Raman signature of disordered pentacene molecules in the film. Finally, the SERS spectrum, via its sensitivity to disorder, is harnessed to develop a diagnostic tool for studying the molecular origins of device performance degradation. An enhancement of the SERS spectrum is seen in those devices that experience degradation in performance as a function of bias stress. On the other hand, no changes in the SERS spectra are observed in devices which feature stable performance. Thus, we confirm that the SERS spectrum can be used as a visualization tool for correlating transport properties to structural changes, if any, in organic semiconductor based devices.

\subsection{SERS from pentacene with other metals}

As was seen in Figure 6.2, the SERS spectrum from the Au-pentacene interface evolves dramatically as the thickness of the Au top film increases. Furthermore, the evolution of the SERS spectrum can be quantified by taking the intensity ratio of any two peaks, as is done in Figure 6.16 (b), for example. Once the peak intensity ratio as a function of thickness for this system has been determined, one immediately sees how the SERS spectrum can be used to measure Au film thickness for subsequent devices. Thus, we have the exciting prospect of the development of an optical spectroscopy based tool 
for characterizing the top metal contact. Now, the effect has, thus far, has only been demonstrated for the Au-pentacene system, However, the SERS effect is also expected from other metals such as $\mathrm{Ag}$ and $\mathrm{Cu}^{22}$ Furthermore, we may expect, by recalling the SERS spectrum of Au on HOPG, an SERS signal from films of organic materials that contain $\mathrm{sp}^{2}$ carbons. If, indeed, a similar effect can be found when using other metals or organic films, then such a tool may be more widely applicable.

Before attempting to generalize metal film thickness sensitivity of the SERS spectrum to other systems, we first revisit the Au-pentance system. For convenience, the SERS spectrum of pentacene as a function of Au contact thickness is provided once again in Figure 6.16 (a). The Au films in these samples were evaporated slowly at $.3 \AA / s$. One immediately notices the drastic changes in the $1160 \mathrm{~cm}^{-1}$ and $1380 \mathrm{~cm}^{-1}$ regions of the spectrum as the Au layer gets thicker. In the former region, the ratio of the two peaks clearly changes as the Au contact gets thicker. In the latter, the $1380 \mathrm{~cm}^{-1}$ grows to dominate the $1371 \mathrm{~cm}^{-1}$ peak. To quantify these changes, the ratios of each pair of peaks is plotted in Figure 6.16 (b) and (c). It is seen that the intensity ratio of the $1160 \mathrm{~cm}^{-1}$ peak to the $1178 \mathrm{~cm}^{-1}$ peak increases till $\sim 3 \mathrm{~nm}$ of the Au layer with a slight drop as the Au thickness is further increased. Beyond $20 \mathrm{~nm}$ of the Au thickness, the ratio remains a constant. In the higher energy region, the intensity ratio of the $1380 \mathrm{~cm}^{-1}$ peak to the 1371 $\mathrm{cm}^{-1}$ peak increases dramatically till $\sim 2 \mathrm{~nm}$, after which it continues to grow slowly through $20 \mathrm{~nm}$. In both cases, the ratio clearly varies smoothly as a function of $\mathrm{Au}$ thickness, with greatest sensitivity for very thin (a few nanometers) Au films. It is evident that the SERS spectrum contains within it a remarkable fingerprint of the thickness of the 
Au top contact. Once the system has been calibrated, one can use the SERS spectrum to measure or monitor the thickness of evaporated Au layer.

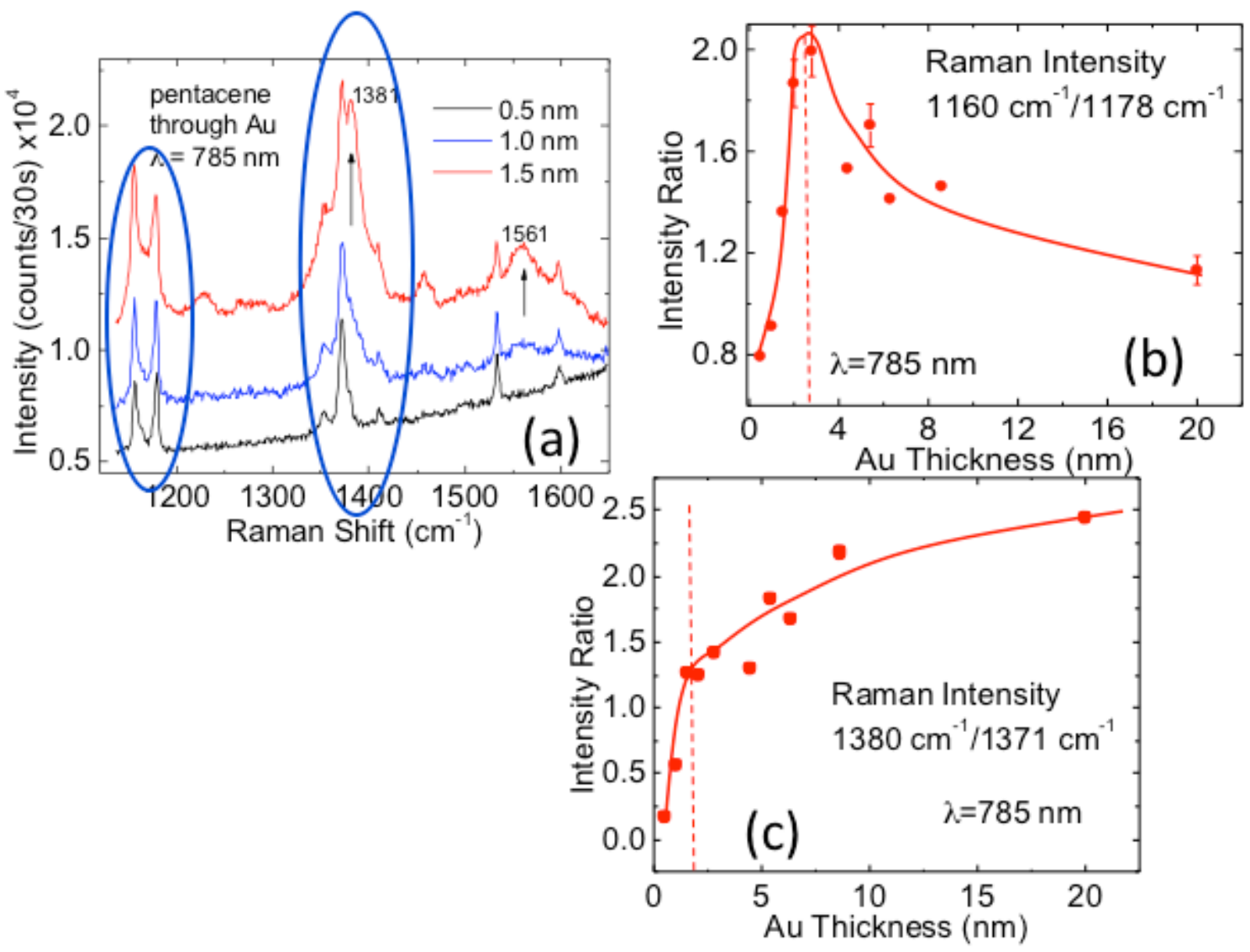

Figure 6.16. (a) The SERS spectrum of pentacene as a function of $\mathrm{Au}$ contact thickenss. (b) The ratio of the Raman intensity of the $1160 \mathrm{~cm}^{-1}$ peak to the $1178 \mathrm{~cm}^{-1}$ peak (measured with the $785 \mathrm{~nm}$ laser line) as a function of the thickness of the Au layer on top of the pentacene film. (c) The ratio of the Raman intensity of the $1380 \mathrm{~cm}^{-1}$ peak to the $1371 \mathrm{~cm}^{-1}$ peak (measured with the $785 \mathrm{~nm}$ laser line) as a function of the thickness of the Au layer on top of the pentacene film. 
Next, we attempt to generalize the previous analysis to systems with metals other than Au. Pentance films were prepared identically to before, but this time, thin layers of $\mathrm{Ag}$ were evaporated on top to complete the specimens. Once again, Ag was evaporated slowly at $.3 \AA / s$ to ensure uniform, thin films. Many sample were prepared, with $\mathrm{Ag}$ layers of various thicknesses. It should be noted that devices with Ag contacts were not prepared, as these specimens were used solely for their SERS spectra. The SERS spectra were measured using a $514 \mathrm{~nm}$ laser. Since Ag surface plasmons emit at higher energies than Au surface plasmons, a higher energy laser must be used to ensure maximum SERS sensitivity. The SERS spectra are shown in Figure 6.17. As with the Au-pentacene specimens, one clearly sees the growth of the SERS spectrum as the Ag layer thickness increases beyond $1 \mathrm{~nm}$. 


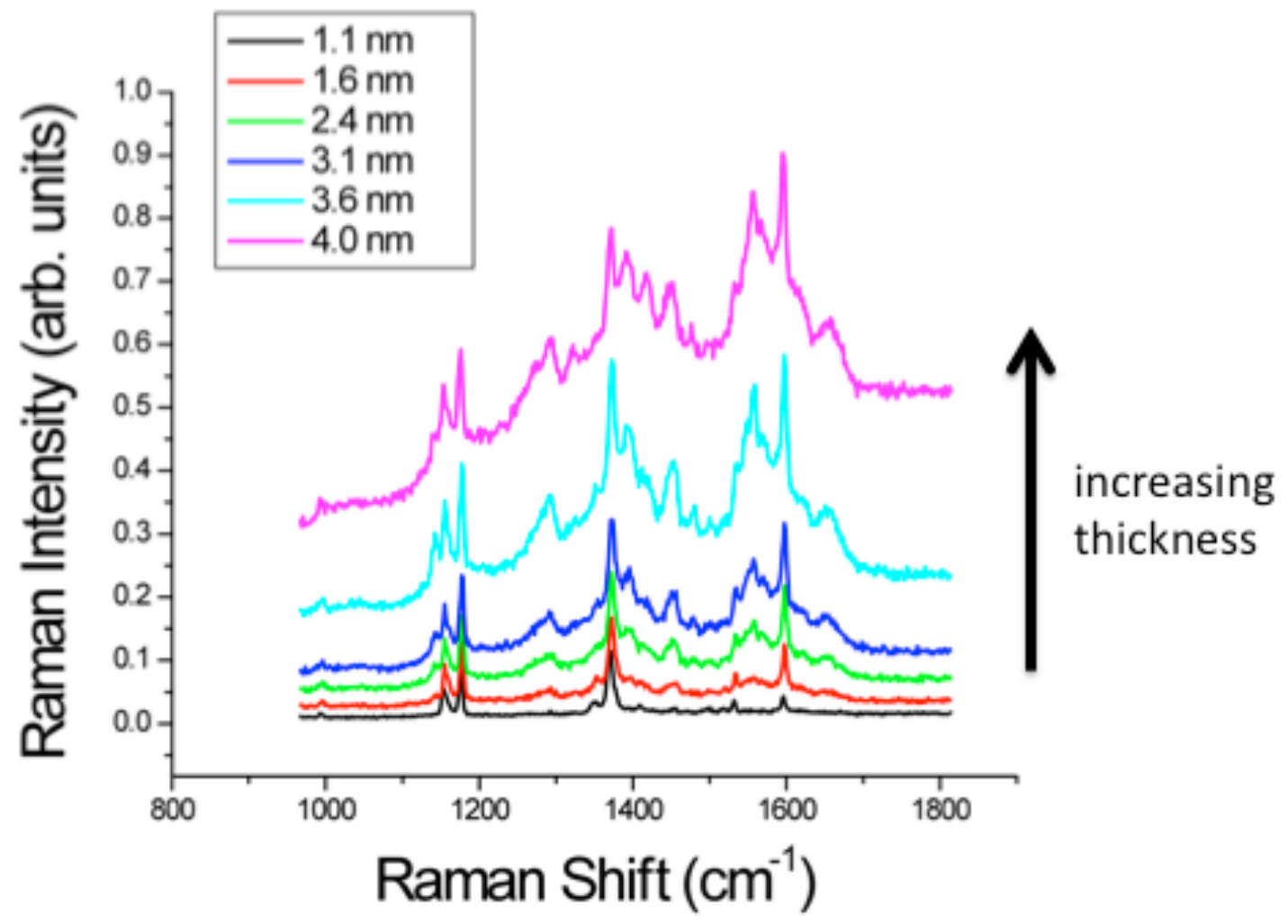

Figure 6.17. The SERS spectra from pentacene films with thin Ag layers evaporated on top.

As the Ag films get thicker and the SERS spectrum evolves, one sees the growth of features at 1400 and $1560 \mathrm{~cm}^{-1}$. While these features clearly change the pentacene spectrum, the spectrum itself keeps its most important features, even more so than it did in the Au-pentacene system. Therefore, one could once again quantify the development of the spectrum by tracking the ratio of peaks. Consequently, it is evident that the SERS features seen from the pentacene OFET geometry are not exclusively limited to the Aupentacene interface. One should expect to see a similar spectrum when depositing other metals that have optical surface plasmon frequencies. 
Thus, a tool that makes use of the correlation of the SERS spectrum to metal contact thickness may be generalized to other systems that feature other metals, such as $\mathrm{Cu}, \mathrm{Ag}$, and $\mathrm{Pt}$.

A system of particular interest may be the metal-dielectric interface formed in the back-end fabrication of modern integrated circuits. copper has become the metal of choice used to form interconnects in such circuits, owing to its high conductivity and electromigration resistance. ${ }^{78}$ Additionally, to minimize capacitive losses, low-k dielectrics are used to isolate the interconnects from each other. Several organic polymers are suitable for use as the low-k dielectric. ${ }^{79}$ To investigate if an SERS spectrum may be detected from a metal-organic dielectric system, $\mathrm{Au}$ was evaporated onto a film of poly(methyl methacrylate) (PMMA), a commonly used organic dielectric. The Raman spectrum of both the pristine PMMA film and PMMA film with the Au contact were then recorded. Sample spectra of each of the two cases are shown in Figure 6.18. It is immediately obvious that there is an SERS effect from the Au-PMMA interface. In fact, the SERS spectrum is somewhat reminicient of the pentacene SERS spectrum, which is not surprising considering how both materials consist mainly of similar carbon-carbon and carbon-hydrogen bonds. Regardless, it is evident that since an SERS spectrum is seen from such interfaces, an SERS based metrology tool for characterizing metal film thicknesses may be developed for these systems. 


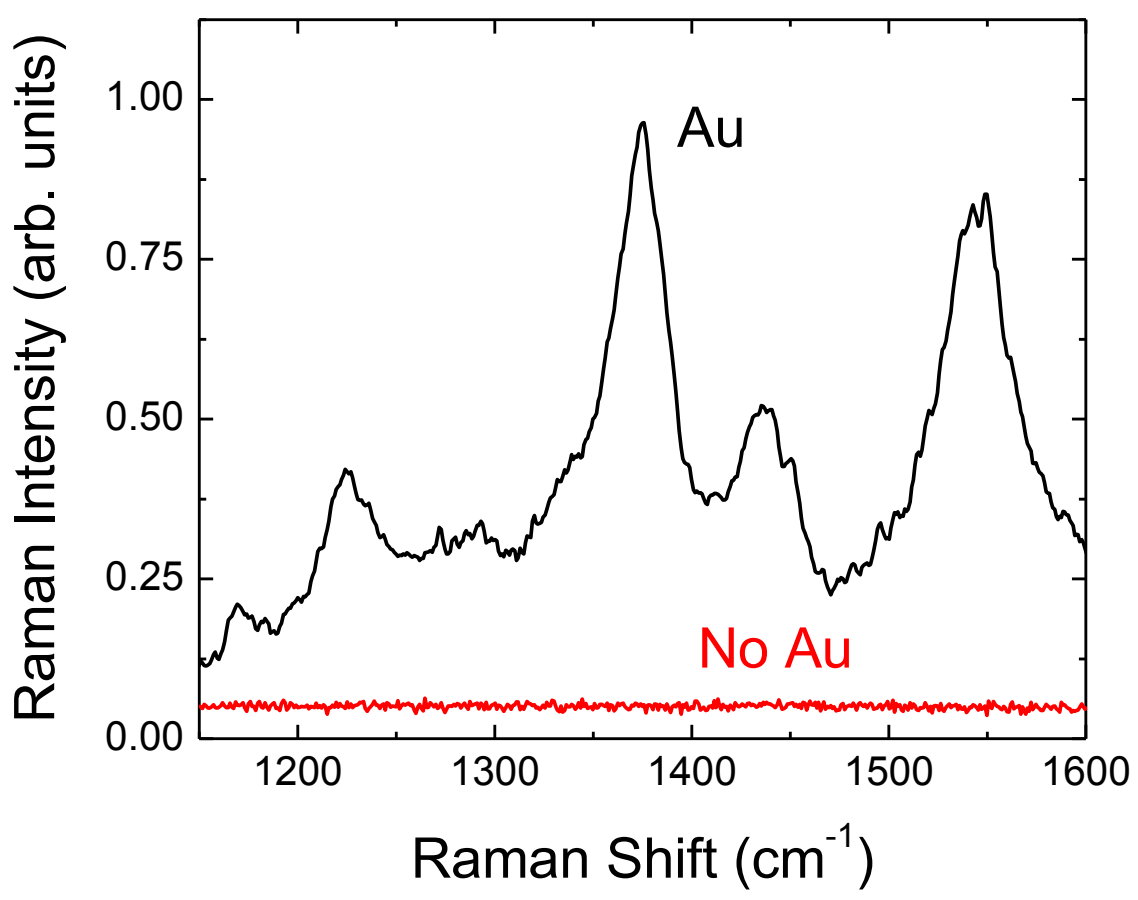

Figure 6.18. The Raman spectrum of a PMMA film taken both in its pristine form (No $\mathrm{Au}$ ) and through a $\mathrm{Au}$ top contact (Au). The SERS spectrum is clearly seen in the latter case. 


\section{OFETs based on Solution Processable Polymers}

\subsection{Diketopyrrolopyrrole Based Polymers}

The design of systems incorporating both donor and acceptor chromophores are of particular interest for applications in field-effect transistors and solar cells. Donor chromophores are defined as those that are electron rich while acceptor chromophores are defined as those that are electron deficient. While the blend of a conjugated polymer donor and a fullerene based acceptor as the active layer is typical in organic solar cells ${ }^{80}$, the large bandgap of most conjugated polymers limits device efficiency. In a landmark paper, Shockley and Queisser showed that for the solar spectrum, the maximum theoretical efficiency for a single junction solar cell occurs at $33.7 \%$ and requires a bandgap of $1.34 \mathrm{eV} \cdot{ }^{81}$ For compounds where the donor and acceptor units are combined on the same monomer, the hybridization of the high-lying HOMO on the donor and the low-lying LUMO on the acceptor results in a donor-acceptor monomer with an unusually low bandgap. ${ }^{82}$ As a result, oligomers and polymers based on donor-acceptor (D-A) moieties have a high degree of tunability of their electronic and optical properties as the relative contribution of the electron-rich and electron-poor components may be controlled.

Diketopyrrolopyrrole (DPP) containing copolymers have recently gained a lot of interest in organic optoelectronics. ${ }^{83,84}$ The DPP subunit structure is shown in Figure 7.1. The strong electron withdrawing nature of the carbonyl functional groups renders the subunit electron deficient. Thus, the DPP subunit serves well as an electron acceptor when combined as a copolymer with less electron deficient moieties. Furthermore, the 
planarity of the DPP skeleton encourages $\pi-\pi$ stacking of its co-polymers. ${ }^{85,86}$ As a result, OFETs fabricated using these polymers can exhibit ambipolar mobilities greater than 1 $\mathrm{cm}^{2} / \mathrm{Vs}^{85,87}$, and solar cells using these polymers in conjunction with fullerene based acceptors can have power conversion efficiencies greater than $5 \% .{ }^{88}$

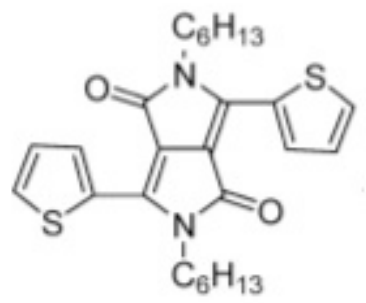

(a)

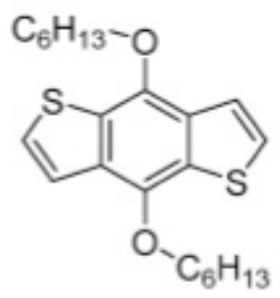

(a)

Figure 7.1. The (a) DDP and (b) BBT subunits. Note the DPP unit here has two thiophene attached to it.

To be used effectively in a D-A polymer, the DPP acceptor unit needs to be paired with a donor unit. While electron rich thiophene and thienothiophene based donor units are commonly used, benzo[1,2-b; 3,4-b] dithiophene (BBT) units have also been used. ${ }^{89,90}$ The BBT subunit is shown in Figure 7.1. The alkoxy functional groups attached to the central aromatic rings have strong electron donating effects. ${ }^{89}$ This chapter reports the application of two DPP-BBT based copolymers in organic OFETs: a phenyl based DPP-BBT and thiophene based DPP-BBT polymer. As seen in Figure 2.6, the two copolymers differ based on the linking moiety between the BBT and the DPP subunits; the phenyl based DPP polymer (PDPP) has a phenyl linking ring while the thiophene based DPP polymer (TDPP) has a thiophene linking ring. Both copolymers are made soluble with aliphatic long side chains attached to both the DPP and BBT subunits. While they 
help with solubility, the side chains do negatively affect charge carrier mobility because they cause an increase in the $\pi$-stacking distance. ${ }^{91}$

The absorption spectra show peaks at $563 \mathrm{~nm}$ and $656 \mathrm{~nm}$ for PDPP-BBT and TDPPBBT films, respectively. ${ }^{92}$ The experimental absorption spectra are shown in Figure 7.2, along with the calculated spectra. The experimental spectra clearly show two absorbance peaks, indicating the D-A nature of the polymers. The lower energy peak corresponds to the charge transfer excitation from the donor moiety to the acceptor moiety. The higher energy peak corresponds to the pi-pi* excitation. The absorption spectra were computed with time dependent DFT using the $3-21 G^{*}$ basis set and three different functionals. The absorbance predicted by the commonly used B3LYP functional shows a single peak. While it predicts the energy of the primary absorbance peak adequately, it completely misses the higher energy peak. Furthermore, although not seen in the absorbance, the B3LYP functional predicts optically silent, sub-bandgap excitations that cannot be reconciled with any experimental results. In fact, the failure of the local functionals, such as B3LYP, in predicting charge transfer energies is well documented. ${ }^{93,94}$ Indeed, if the excitations in the DPP polymers have a charge transfer nature, which would be the case for a donor-acceptor system, a functional that better incorporates long-range effects should give better results. The CAM-B3LYP functional incorporates a long-range correction to the B3LYP functional by properly incorporating the long-range exchange potential. ${ }^{95}$ It has been shown to be better at computing intramolecular charge transfer excitations. ${ }^{96}$ As shown in Figure 7.2, the absorbance predicted using the CAM-B3LYP functional does have the second, higher energy peak. While the peak is weak, an unmistakable shoulder in the main absorbance peak is seen at 4.0 and $3.3 \mathrm{eV}$ for PDPP 
and TDPP, respectively. While CAM-B3LYP does clearly show the dual absorbance peak nature that a donor-acceptor based polymer should have, it does not re-create the shape of the experimental absorbance well. To improve upon the theoretical spectra, the M06HF functional is optimized for charge transfer excitation by incorporating the full Hartree-Fock exchange. ${ }^{97}$ Here, the absorbance predicted using the M06HF functional has two distinct peaks, and does a remarkably good job re-creating the shape of the absorbances. Of course, it tends to over-predict energies, but that is the expected cost when using large amount of Hartree-Fock exchange. ${ }^{98}$ Overall, the better performance of functionals optimized for charge transfer excitations gives strong theoretical support that the DPP polymers behave as donor-acceptor systems.

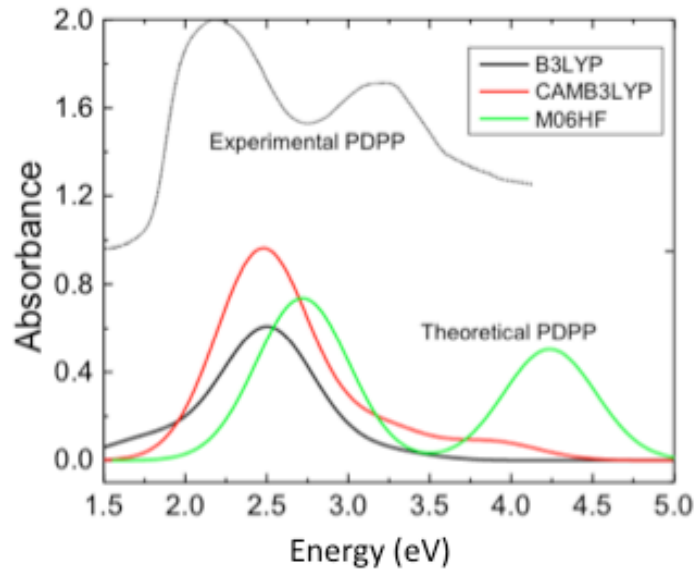

(a)

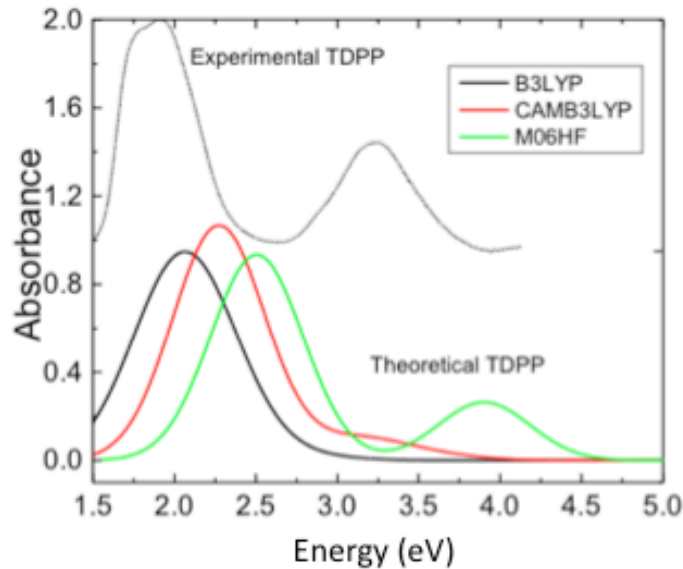

(b)

Figure 7.2. The experimental and theoretical absorbances of (a) PDPP and (b) TDPP.

The transistor characteristics of the DPP copolymers correlate with the morphology of the films and capacitance-voltage characteristics of their corresponding MIS diode structures. Both copolymers exhibit p-type transistor characteristics with hole mobilities 
in the range $10^{-3}-10^{-4} \mathrm{~cm}^{2} / \mathrm{Vs}$ for spun-cast films depending upon the molecular weight. No degradation in device performance was observed, even after operating the device in air.

A total of three DPP-based copolymer samples were used: one PDPP sample (molecular weight $9700 \mathrm{~g} / \mathrm{mol}$ ) and two TDPP samples, a lower molecular weight version (sample A, molecular weight $3510 \mathrm{~g} / \mathrm{mol}$ ) and a higher molecular weight version (sample $\mathrm{B}$, molecular weight $5080 \mathrm{~g} / \mathrm{mol}$ ). Solutions of the samples were prepared by dissolving the polymer in Chloroform at a concentration of $5 \mathrm{mg} / \mathrm{ml}$ and filtering the solution through a $.22 \mu \mathrm{m}$ PTFE filter. The solutions were spun-cast on to OTS treated $\mathrm{Si} / \mathrm{SiO}_{2}$ substrates at $2000 \mathrm{rpm}$ to give films $\sim 20 \mathrm{~nm}$ thick. Attempts were made to use solutions of higher concentration (up to $\sim 10 \mathrm{mg} / \mathrm{ml}$ ), but the DPP materials were found to be only marginally soluble at those concentrations. Films spun-cast from these more concentrated solutions had precipitates and were generally found to be of lower quality. Attempts were also made to dissolve the DPP materials in dichlorobenzene. It has been shown in other OS systems that using higher boiling point solvents yields better device performance. ${ }^{99}$ However, the DPP copolymers were not sufficiently soluble in dichlorobenzene.

The current-voltage characteristics of solution processed PDPP-BBT and TDPP-BBT (sample A) OFETs on OTS treated $\mathrm{Si} / \mathrm{SiO}_{2}$ substrates are shown in Figure 7.3 and Figure 7.4. Both the PDPP-BBT and the TDPP-BBT devices are p-type and show typical fieldeffect transistor behavior such as good modulation of drain-source current by gate voltage and drain-source current saturation at high gate voltages. There is almost no hysteresis in the output characteristics of the OFETs as shown in Figure 7.3(a). The leakage current was at least three-orders of magnitude smaller than the measured currents. The current- 
voltage characteristics of the TDPP-BBT OFETs shown here have a $W / L$ ratio of 10 and the PDPP-BBT devices shown here have a $W / L$ ratio of 20 . The OFET characteristics were also measured from the higher molecular TDPP-BBT sample as shown in Figure 7.5. The transistor characteristics were measured from at least 20 devices with different $W / L$ ratios; almost all devices show similar characteristics.

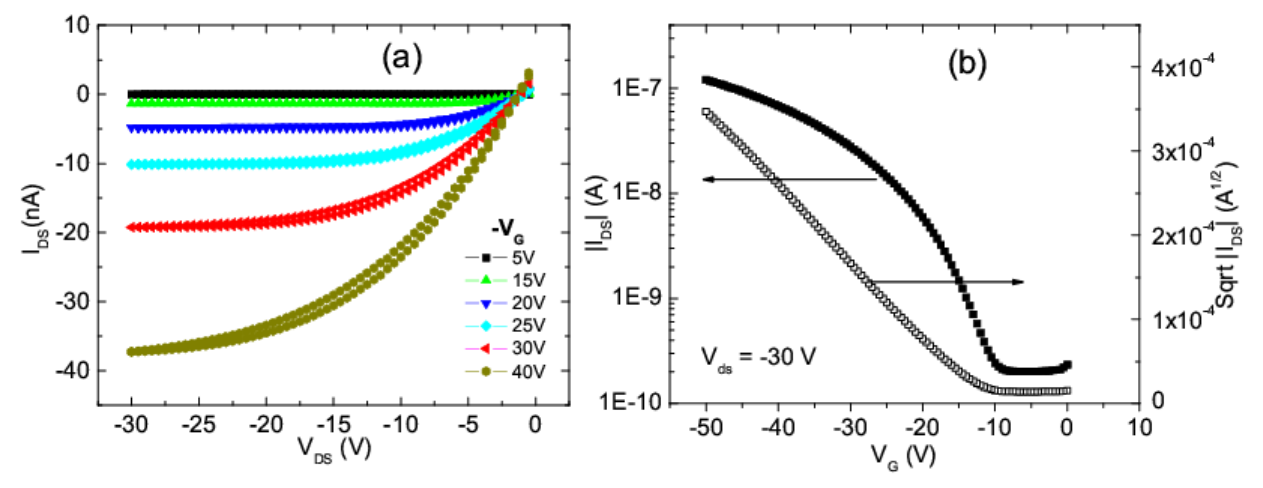

Figure 7.3. (a) Output and (b) transfer characteristics of PDPP-BBT OFET
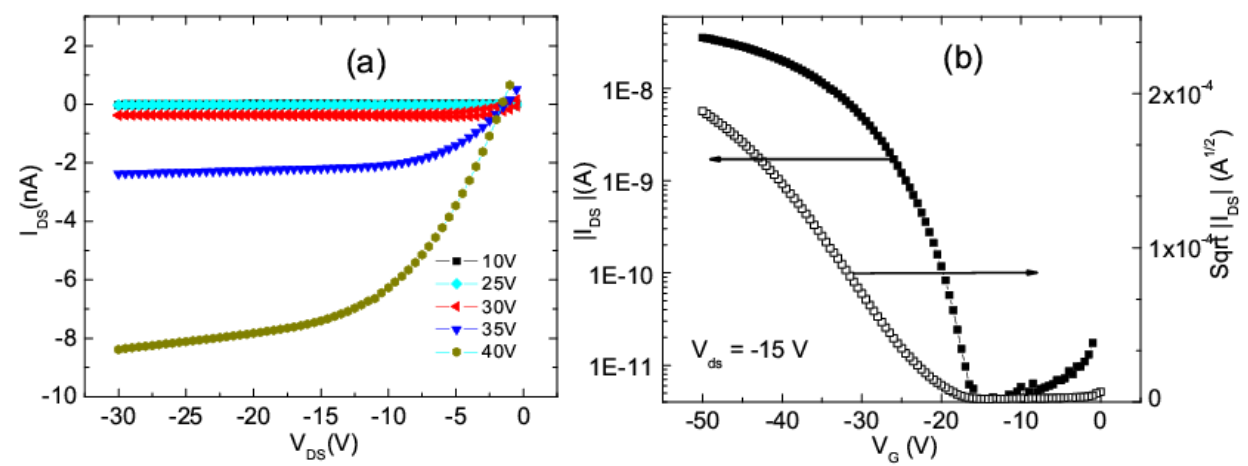

Figure 7.4. (a) Output and (b) transfer characteristics of TDPP-BBT (sample A) OFET. 


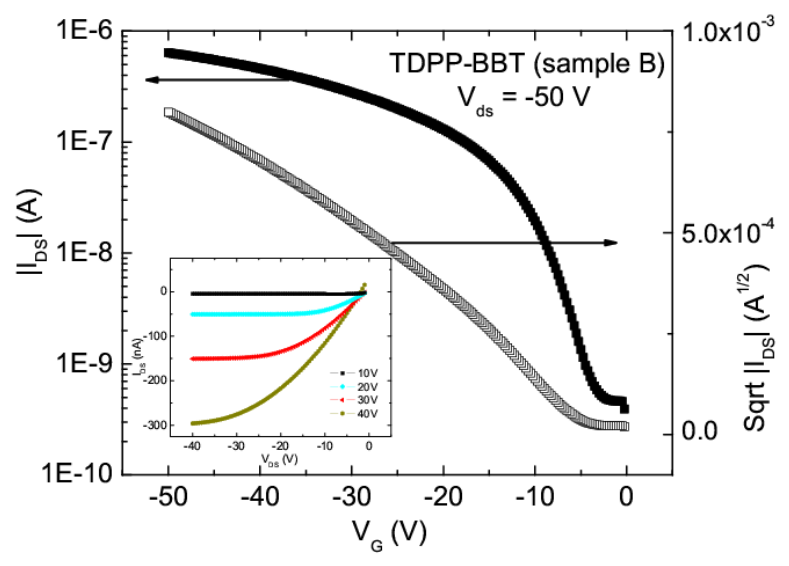

Figure 7.5. Transfer characteristics of TDPP-BBT (sample B) OFET. The inset shows the output characteristics from the same device

Device parameters such as charge carrier mobility, On/Off current ratio, and threshold voltage were estimated using the standard saturation regime current-voltage characteristics of field-effect transistors (eq. 2.7). The square root of the drain-source current as a function of the gate voltage is plotted in Figure 7.3 (b) and Figure 7.4 (b). A linear fit was applied to the saturation region to obtain the slope and from Eq. 2.8. the hole mobilities were found to be $1 \times 10^{-3} \mathrm{~cm}^{2} / \mathrm{Vs}$ for PDPP-BBT and $3 \times 10^{-4} \mathrm{~cm}^{2} / \mathrm{Vs}$ for TDPP-BBT (sample A). The On/Off ratios for both OFETs were $\sim 10^{3}$ for all samples. Sample B shows a considerably higher mobility of $7 \times 10^{-3} \mathrm{~cm}^{2} / \mathrm{Vs}$ as determined from the transfer characteristics of Figure 7.5. The results are tabulated in table 7.1. Threshold voltages were determined by extrapolating the linear fit to zero drain-source current. PDPP-BBT was found to have a threshold voltage of -11V, TDPP-BBT (sample A) has a threshold voltage of $-15 \mathrm{~V}$, and TDPP-BBT (sample B) was found to have a threshold voltage of $-5 \mathrm{~V}$. The deviation of threshold voltages from being zero indicates that mobile holes accumulate in the PDPP-BBT/SiO 2 interface at a lower (in magnitude) gate voltage 
than they do at the TDPP-BBT/SiO 2 (sample B) interface. This result is consistent with the MIS characteristics, which show a slightly higher density of interface traps and the accumulation region occurring at a higher (in magnitude) gate voltage in TDPP-BBT.

We attribute the lower mobility in TDPP-BBT (sample A) to the actual film morphology due to its lower molecular weight compared to PDPP-BBT. In regioregular poly-(3-hexylthiophene) the OFET charge carrier mobilities increase by more than three orders of magnitude when the molecular weight increases by three orders of magnitude. ${ }^{100}$ Our results point to the same trend. When the molecular weight of TDPPBBT is increased by a factor of $\sim$ three, the charge carrier mobility is found to increase by an order of magnitude, as seen in Figure 7.5.

Table 7.1. Electrical characteristic parameters for DPP copolymer based OFETs.

\begin{tabular}{lcccc}
\hline & $\begin{array}{c}\text { Molecular } \\
\text { Weight } \\
(\mathbf{g} / \mathbf{m o l})\end{array}$ & $\begin{array}{c}\text { Mobility } \\
\left(\mathbf{c m}^{2} / \mathbf{V s}\right)\end{array}$ & $\begin{array}{c}\text { Threshold } \\
\text { Voltage } \\
(\mathbf{V})\end{array}$ & $\begin{array}{c}\text { On/Off } \\
\text { ratio }\end{array}$ \\
\hline PDPP-BBT & 9700 & $1 \times 10^{-3}$ & -11 & $\sim 10^{3}$ \\
$\begin{array}{l}\text { TDPP-BBT } \\
\text { (sample A) }\end{array}$ & 3510 & $4 \times 10^{-4}$ & -15 & $\sim 10^{3}$ \\
$\begin{array}{l}\text { TDPP-BBT } \\
\text { (sample B) }\end{array}$ & 5080 & $7 \times 10^{-3}$ & -5 & $\sim 10^{3}$ \\
\hline
\end{tabular}

Since these diketopyrrolopyrrole-based polymers have been shown to be promising candidates for the fabrication of OFETs, it is natural to ask if a Raman scattering based tool, similar to the one developed for pentacene OFETs, may be applied to transistors fabricated using these materials. In searching for a similar SERS effect, we will need to once again use a $785 \mathrm{~nm}$ near infrared laser. As discussed in chapter 6, this wavelength will allow for the excitation of surface plasmons at the Au-semiconductor interface. 
However, since, the TDPP polymer has a non-zero absorbance at $785 \mathrm{~nm}(1.57 \mathrm{eV})$, as seen in Figure 7.2, it tends to burn when irradiated with a laser at this wavelength at power densities necessary for Raman spectroscopy. Therefore, we limit our Raman discussion to the PDPP polymer, and use its results as representative for the DPP polymer class.

An intensity profile of a Raman linescan of a PDPP-BBT OFET structure is shown in Figure 7.6. An individual Raman spectrum of PDPP-BBT is shown in Figure 7.6 (c). PDPP-BBT has a number of Raman modes in the $1100-1700 \mathrm{~cm}^{-1}$ region. A recent experimental and theoretical work on pyridyl substituted DPP clarifies the origin of many of the peaks that we observe. ${ }^{101}$ The $1600 \mathrm{~cm}^{-1}$ Raman peak is from the intra-ring C-C stretch motion (ring breathing) of the phenyl group. This is observed in all phenyl-based conjugated system. ${ }^{102}$ The $1550 \mathrm{~cm}^{-1}$ and $1364 \mathrm{~cm}^{-1}$ peaks originate from the $\mathrm{C}=\mathrm{C}$ and $\mathrm{C}$ C stretch of the central butadiene moiety, respectively. ${ }^{101}$ The $1200 \mathrm{~cm}^{-1}$ Raman frequency is from a C-H bending motion of the phenyl ring ${ }^{103}$, which is not observed in TDPP-BBT.

We note that there is no SERS effect observed in this system. The Raman spectra measured from both the channel and contact regions of the PDPP OFET have the same shape with no evidence of selective peak enhancements or new peaks being formed. As we have previously shown the SERS effect in the pentacene-based system to be indicative of disorder in the semiconductor layer caused by gold evaporation, we believe the lack of an SERS effect in the PDPP-based system shows that evaporated gold does not create the same disorder in PDPP semiconductor. 


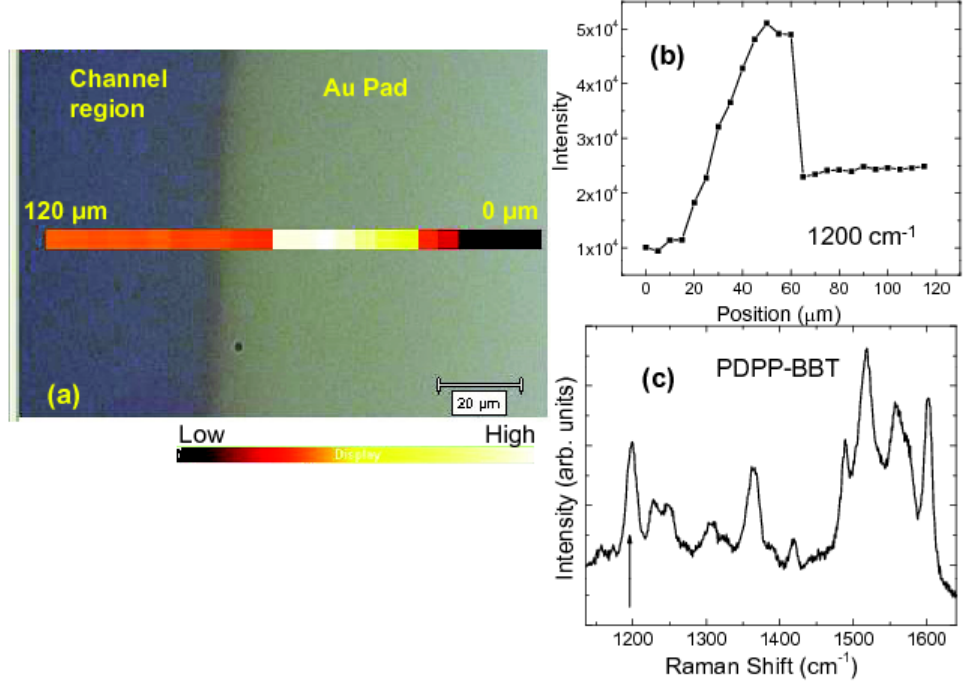

Figure 7.6. (a) Optical image of the channel/contact region in PDBB-BBT OFET. (b) Intensity profile of the $1200 \mathrm{~cm}^{-1}$ Raman peak, corresponding to the color image in (a).(c) Raman spectrum of PDPP-BBT.

Figure 7.7 shows the intensity profile of the Raman spectra in the $1150-1600 \mathrm{~cm}^{-1}$ range before and after biasing a PDPP-BBT OFET. Data sets 0 and 24 correspond to 0 $\mu \mathrm{m}$ and $120 \mu \mathrm{m}$ positions, respectively. Intensity enhancement of the Raman peaks is seen close to the edge of the Au contact and the channel region. There is almost no change observed in the Raman maps before and after biasing, indicating no induced structural changes in the polymer layer upon the application of the electric field. Raman measurements were performed on more than 10 devices, and they all showed a similar behavior. Furthermore, repeated electrical measurements in ambient conditions over a few weeks showed no degradation in device performance. 

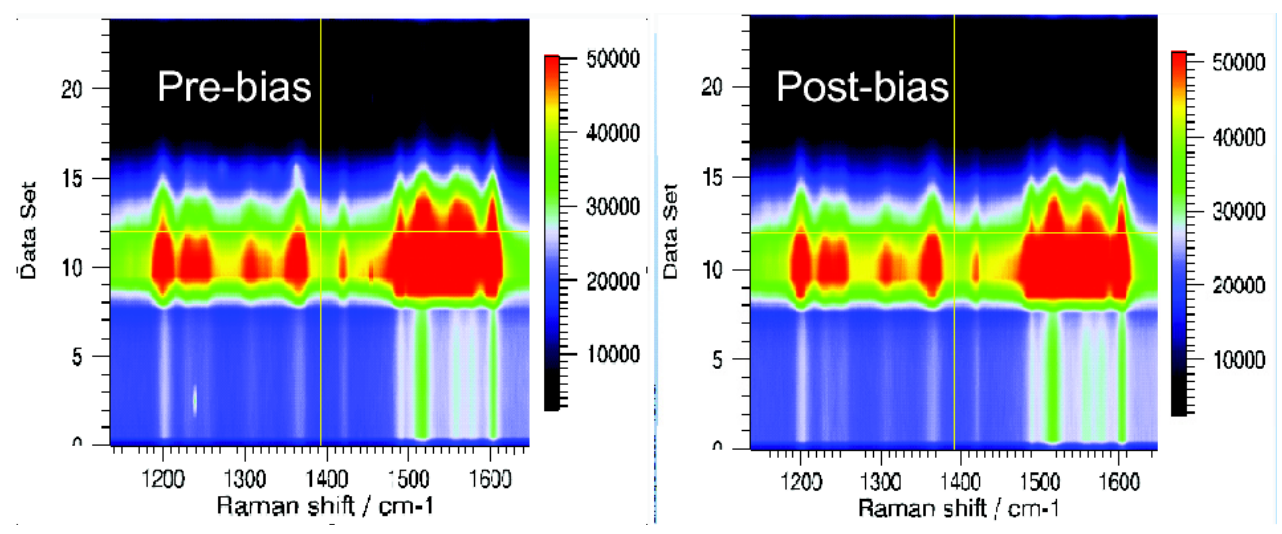

Figure 7.7. Raman maps of PDPP-BBT along the channel/contact region before and after biasing the OFET.

We compare the Raman maps of PDPP-BBT with $\mathrm{SiO}_{2}$ based pentacene OFETs (Figure 6.14), which were also measured before and after biasing. Comparing the two Raman maps in Figure 6.14, one clearly sees changes in the intensity profiles. These changes have been discussed in section 6.4. As mentioned before, these changes are irreversible. Unlike PDPP-BBT, pentacene OFETs show a reduction in mobilities after a few weeks when the devices have been operated many times.

In summary, diketopyrrolopyrrole-based copolymers, PDPP-BBT and TDPPBBT, containing donor-acceptor structural units are promising candidates for solutionprocessed p-channel OFETs. The transistor characteristics show a good modulation of drain-source current by gate voltage with almost no hysteresis in the current-voltage output characteristics. Charge carrier mobilities $\sim 10^{-3} \mathrm{~cm}^{2} / \mathrm{Vs}$ in spin-coated PDPPBBT/TDPP-BBT OFETs show that these DPP-based copolymers are excellent candidates for application in organic electronics. 


\subsection{Polythiophene Based Polymers}

While solution processable donor-acceptor systems, such as those based on the DPP moiety, are promising candidates for organic devices, a much greater amount of work has been done with simpler homopolymer systems. Specifically, the compound poly(3hexylthiophene) (Figure 7.8) has been used extensively in both OFETs and OPVs. Note that the $\mathrm{P} 3 \mathrm{HT}$ monomer is nothing more than thiophene rings with solubilizing alkyl side chains. Due to its exceptional planarity, P3HT molecules have a tendency to stack via $\pi$ $\pi$ interactions. ${ }^{104}$ This stacking leads to films with high degrees of molecular order. As a result, P3HT based OFETs can have mobilities of $0.1 \mathrm{~cm}^{2} \mathrm{~V}^{-1} \mathrm{~s}^{-1} .{ }^{15}$ In fact, when fabricated with Pt electrodes and organic dielectric films, a mobility of $0.4 \mathrm{~cm}^{2} \mathrm{~V}^{-1} \mathrm{~s}^{-1}$ has been reported. ${ }^{105}$ Furthermore, P3HT, along with the fullerene-based [6-6]-phenyl $\mathrm{C}_{61}{ }^{-}$ butryric acid methyl ester (PCBM), is commonly used in bulk heterojunction organic solar cells with efficiencies in the $4-5 \%$ range. ${ }^{106,107}$

As promising as a system $\mathrm{P} 3 \mathrm{HT}$ is, it is substantially limited by its susceptibility to oxidation. It is well known that $\mathrm{P} 3 \mathrm{HT}$ is very susceptible to oxidative doping, and thus P3HT-based devices show poor performance under ambient conditions. ${ }^{15}$ P3HT is an exceptionally electron rich polymer as the solubilizing alkyl side chains effectively donate electron density to the already electron-rich planar thiophene rings. This high electron density is what leads to the low ionization potential of P3HT, and the resultant susceptibility to oxidation. In fact, it has been shown that the oxidative destruction of P3HT is initiated via the oxidation of the alkyl side chains, and that unsubstituted polythiophenes are not susceptible to similar oxidation. ${ }^{108}$ Polythiophenes substituted with electron withdrawing alkoxy side chains are also resistant to oxidation, further 
confirming the alkyl side chain culpability in the incompatibility of P3HT with oxygen. $^{109}$

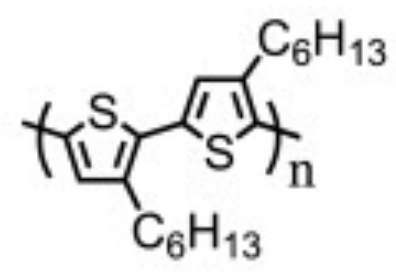

rr-P3HT

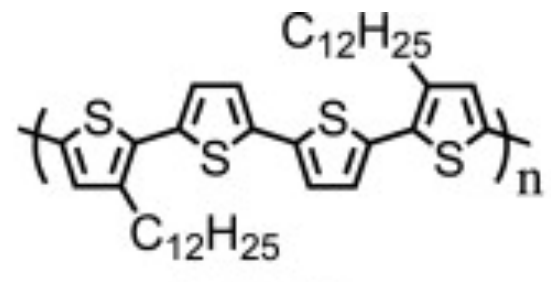

PQT-12

Figure 7.8. The structures of $\mathrm{P} 3 \mathrm{HT}$ and $\mathrm{PQT}$.

Since the solubilizing alkyl side chains of P3HT are responsible for its limitations in ambient conditions, it is reasonable to ask if one can modify them without sacrificing the conduction ability of the thiophene backbone. In order to do this, the delocalization of charge in the polymer must be reduced via a reduction in the conjugation length. ${ }^{15}$ In fact, this has been accomplished in the synthesis of poly-(3,3-dialkyl-quaterthiophene) (PQT), a thiophene based polymer that has solubilizing alkyl side chains on only some of its thiophene rings. ${ }^{110}$ The structure of PQT is given in Figure 7.8. The presence of unsubstituted thiophene allows PQT more rotational freedom, thus cutting down on $\pi$ conjugation and lower electron density. While some amount of planarity is sacrificed, PQT maintains is ability to $\pi$ stack, and thus yields OFETs with mobilities up to $0.14 \mathrm{~cm}^{2}$ $\mathrm{V}^{-1} \mathrm{~S}^{-1}$ in ambient conditions. ${ }^{111}$

Thus, we can use the PQT system to study the optical and electrical characteristics of polythiophene based OFETs without being limited with incompatibility of P3HT with ambient conditions. While extensive work has been done in the electrical characterization of polythiophene based OFETs and OPVs, there has also been work done in the optical 
characterization of such devices. For example, Raman scattering has been used to determine the degree of ordered vs. disordered P3HT in P3HT/PCBM films. ${ }^{12}$ It is evident that polythiophene is a promising solution processable alternative to pentacene for a similar optical characterization techniques as used in chapters 6 and 7. Since PQT and P3HT differ only in the nature of the alkyl side chains, the Raman spectra of the two will be more or less identical; at least as far as the intra-ring vibrations are concerned

PQT films were prepared for OFETs on $\mathrm{Si}^{++} / \mathrm{SiO}_{2}$ substrates. The procedure for cleaning and treating the substrates is given in chapter 3. PQT (American Dye Source) was dissolved in Dichlorobenzene (ReagentPlus 99\%, Sigma Aldrich) at a concentration of $10 \mathrm{mg} / \mathrm{ml}$. The solution was found to be a cloudy, brick red color, indicating incomplete solubility of the PQT in the solvent. The solution was heated to $100^{\circ} \mathrm{C}$ where it turned to a bright candy-red color. As the solution was allowed to cool, it became cloudy again, further confirming that the PQT is completely soluble at this concentration only at elevated temperatures. Clearly, the solution would have to be heated during use to ensure adequate solubility of the PQT. After re-heating the solution, it was quickly filtered through a $.22 \mu \mathrm{m}$ Teflon filter. While the solution was still warm, it was spun-cast at $3000 \mathrm{rpm}$ by dropping $\sim .3 \mathrm{ml}$ of solution onto both untreated and OTS treated substrates. A purple colored film was seen to form. Attempts were also made to spin-coat at other speeds ranging from $1500 \mathrm{rpm}$ to $5000 \mathrm{rpm}$. It was found that films were not formed at lower speeds, and that very thin films were formed at higher speeds. It should be noted that dichlorobenzene has much higher boiling point than the chloroform $\left(180^{\circ} \mathrm{C}\right.$ vs. $62^{\circ} \mathrm{C}$ ) that was used to spin-coat the DPP materials discussed earlier, and as such, is 
more difficult to spin-coat with. $3000 \mathrm{rpm}$ was found to be good balance between the higher and lower spin speeds.

After spin-coating, the films were annealed at $140^{\circ} \mathrm{C}$ for 30 minutes. PQT undergoes crystalline-to-liquid crystalline and liquid crystalline-to-isotropic phase transitions at $\sim 120^{\circ} \mathrm{C}$ and $\sim 140^{\circ} \mathrm{C}$, respectively. ${ }^{111}$ When annealed at $120^{\circ} \mathrm{C}$ to $140^{\circ} \mathrm{C}$, PQT films have been shown to have excellent $\pi-\pi$ packing. ${ }^{113}$ After annealing, the films were almost completely transparent, with a greenish tint, confirming that the molecular ordering, and thus, electronic structure of the film had changed.

Top contacts of Au were evaporated onto the films to complete OFET fabrication. $\sim 30$ $\mathrm{nm}$ of $\mathrm{Au}$ was thermally evaporated at a rate of $\sim 3 \AA / \mathrm{s}$. The transfer and output characteristics of a device fabricated on an untreated substrate are shown in Figure 7.9 and Figure 7.10, respectively. The output curves show good modulation with a clear saturation regime, and the transfer curve show clear switching behavior with a low threshold voltage, all characteristics of a decent transistor. Common device parameters were estimated using eq. 2.7. Charge carrier mobility was found to be $3.70 \times 10^{-4} \mathrm{~cm}^{2} / \mathrm{Vs}$, the On/OFF ratio was $2.5 \times 10^{2}$, and the threshold voltage was $2.0 \mathrm{~V}$. These initial unoptimized devices were fabricated on untreated substrates (i.e. no SAM layer) to confirm that this system would yield usable devices. Later, devices were fabricated on OTS treated substrates to maximize performance. 
Table 7.2. Electrical characteristic parameters for PQT OFETs.

\begin{tabular}{cccc}
\hline & $\begin{array}{c}\text { Mobility } \\
\left(\mathbf{c m}^{2} / \mathbf{V s}\right)\end{array}$ & $\begin{array}{c}\text { Threshold Voltage } \\
(\mathbf{V})\end{array}$ & On/Off ratio \\
\hline $\begin{array}{c}\text { PQT } \\
\text { without } \\
\text { OTS }\end{array}$ & $3.70 \times 10^{-4}$ & -2.0 & $\sim 10^{2}$ \\
$\begin{array}{c}\text { PQT } \\
\text { with OTS }\end{array}$ & $2.80 \times 10^{-3}$ & & $\sim 10^{3}$ \\
\hline
\end{tabular}

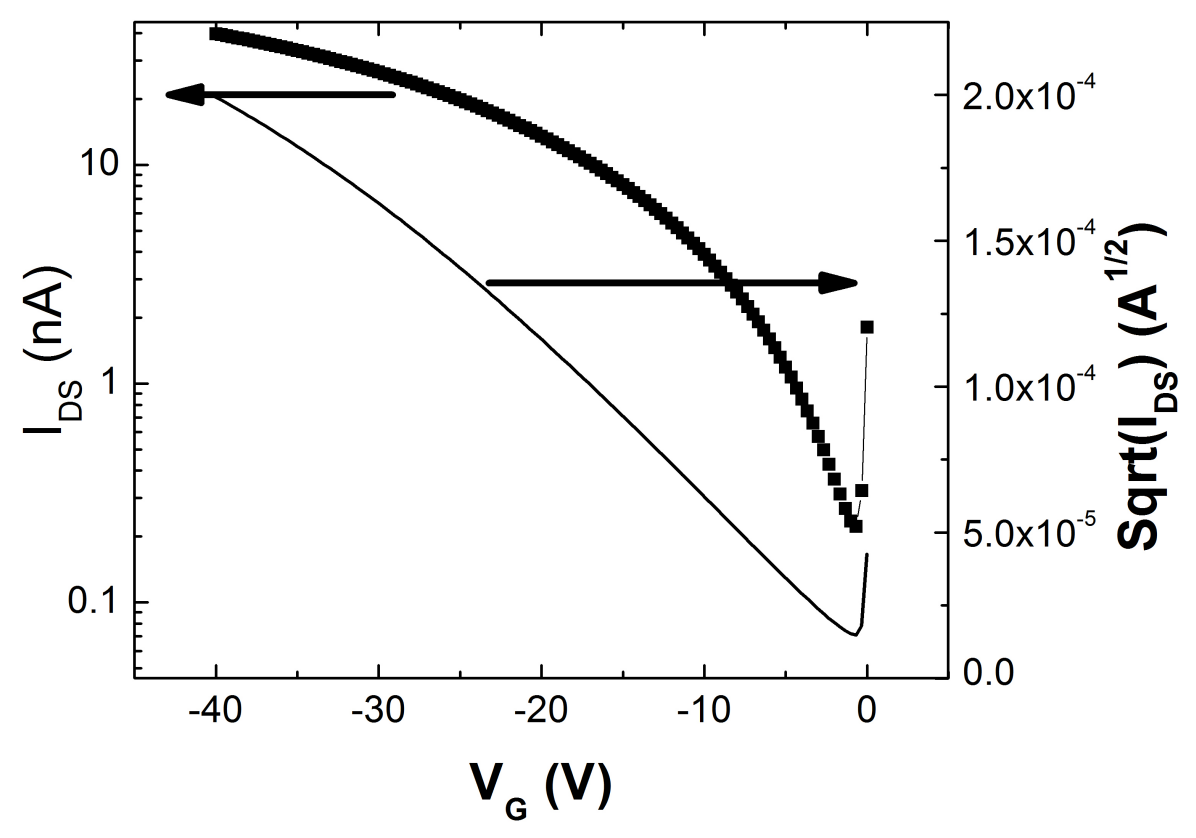

Figure 7.9. The transfer characteristics of an PQT OFET 


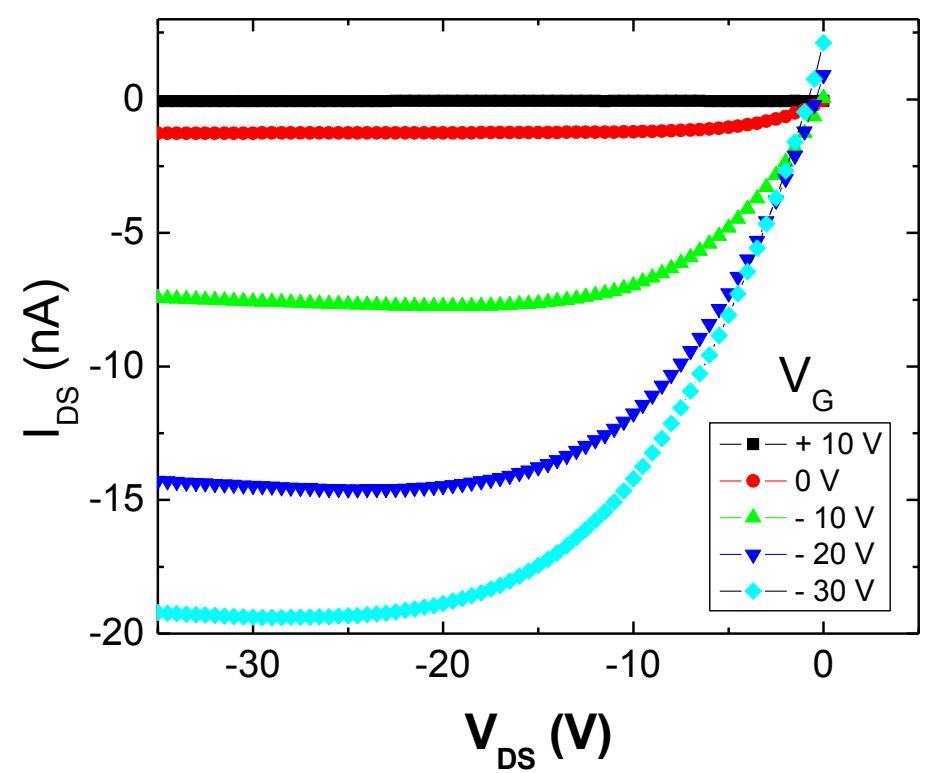

Figure 7.10. The output characteristics of an PQT OFET

The Au top contact in these PQT OFETs creates a metal-semiconductor interface similar to the Au-pentacene interface through which an SERS spectrum was observed (as discussed in chapters 6 and 7. The Raman spectrum of PQT was measured in both the channel and contact regions, using identical laser intensities and exposure times. The two spectra are plotted in Figure 7.11. It is immediately apparent that there is a strong enhancement of the Raman intensities when the spectrum is measured through the $\mathrm{Au}$ contact. The different relative enhancements of different peaks, combined with the appearance of new peaks point to an SERS effect, as was seen in the Au-pentacene system. In fact, several of the most distinguishing features of the former spectrum are seen here in the PQT spectrum as well. As both pentacene and PQT are highly conjugated, aromatic ring based systems, this is perhaps not surprising. 


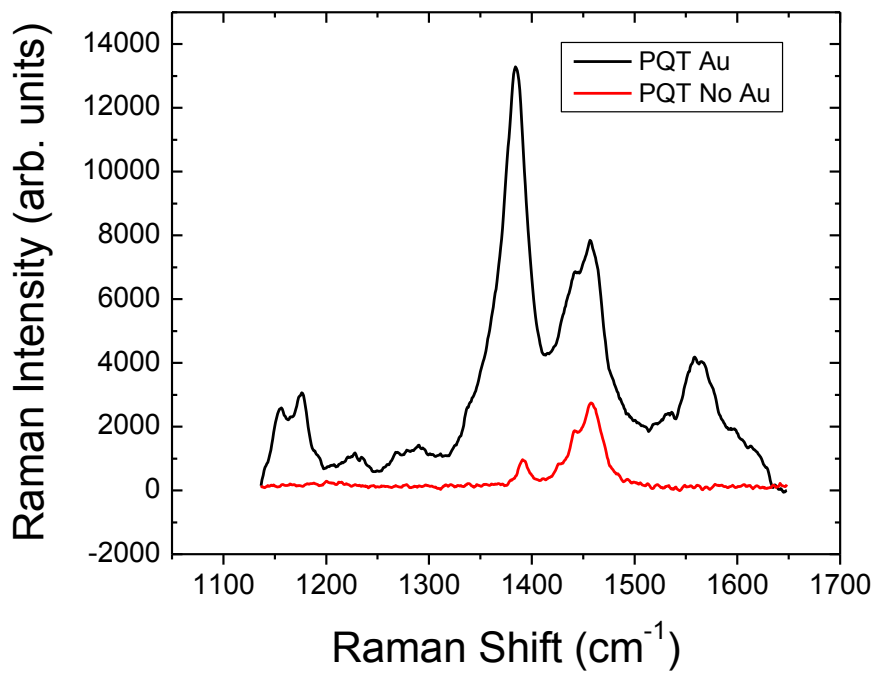

Figure 7.11. The Raman spectrum of PQT as measure from the channel (PQT no $\mathrm{Au}$ ) and contact $(\mathrm{PQT} \mathrm{Au})$ regions of a PQT OFET. It is apparent that the spectrum from the contact region exhibits the characteristics of SERS

The primary features of the pristine PQT spectrum are the two peaks at $1450 \mathrm{~cm}^{-1}$ and $1380 \mathrm{~cm}^{-1}$. The two peaks correspond to $\mathrm{C}=\mathrm{C}$ stretch mode and the $\mathrm{C}-\mathrm{C}$ intra-ring stretch mode, respectively. ${ }^{112}$ The higher energy peak splits into two peaks, the relative intensities of which are determined by the degree of order in the film. These two features are still seen in the SERS spectrum, but several other peaks join them. A new broad peak in the $1560^{-1} \mathrm{~cm}$ region is seen, along with a pair of peak in the 1160 to $1180 \mathrm{~cm}^{-1}$ region. Furthermore, the $1380 \mathrm{~cm}^{-1}$ is strongly enhanced relative to the $1450 \mathrm{~cm}^{-1}$ peak. In order to gain further insight into the nature of these new peaks, the Raman spectrum of polythiophene was simulated using DFT. The method used is detailed in chapter 3 . 
The calciulated Raman spectrum of pristine polythiophene is shown in Figure 7.12. The structure used to simulate the spectrum is shown in Figure 7.13. It should be noted that this structure is a generic polythiophene and thus, can be used for both PQT and P3HT. Others have used a similar simplification. ${ }^{112}$ The dominant peak at $1460 \mathrm{~cm}^{-1}$ corresponds to the experimental peak at $1450 \mathrm{~cm}^{-1}$. Other theoretical peaks at 1550,1100 , and $1400 \mathrm{~cm}^{-1}$ offer clues as to the origins of the peaks in the SERS spectrum. The peak at $1560 \mathrm{~cm}^{-1}$ is due to an anti-symmetric $\mathrm{C}=\mathrm{C}$ stretch. The peaks at $1100 \mathrm{~cm}^{-1}$ are caused by $\mathrm{C}-\mathrm{H}$ bend modes. Lastly, the peak at $1400 \mathrm{~cm}^{-1}$ corresponds to an intra-ring $\mathrm{C}-\mathrm{C}$ stretch, as expected. It is clear that the peaks seen in the SERS spectrum all correspond to theoretically predicted Raman active vibrations. The SERS spectrum is showing sensitivity to vibrational modes in the polythiophene that are undetectable via regular Raman spectroscopy. However, the shape of the simulated pristine PQT spectrum by itself clearly cannot account for the shape of the SERS spectrum.

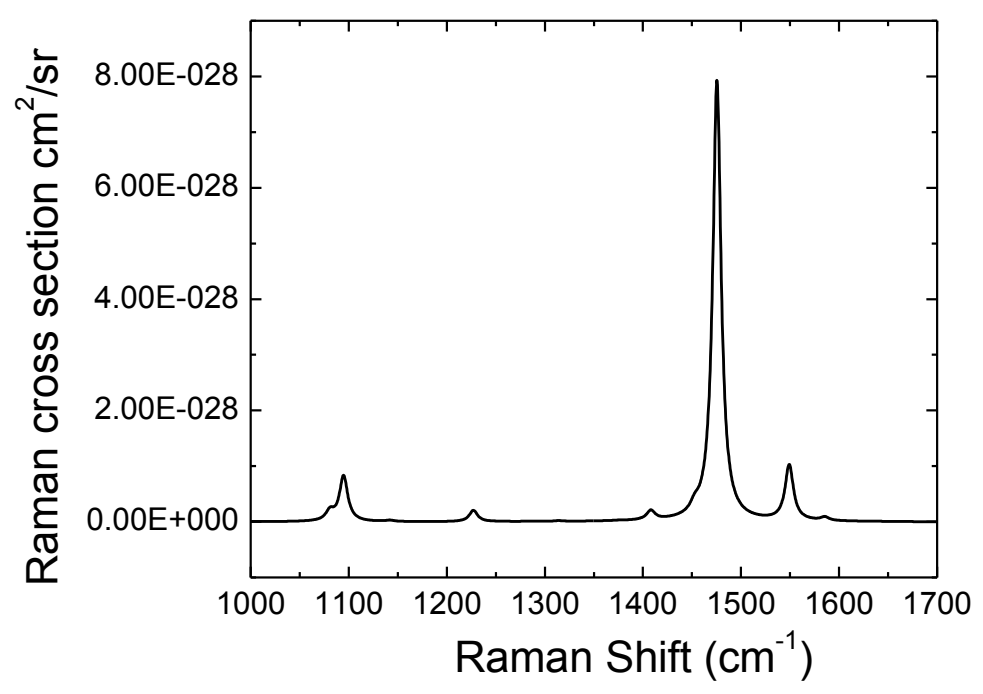

Figure 7.12. The calculated Raman spectrum of pristine PQT. 


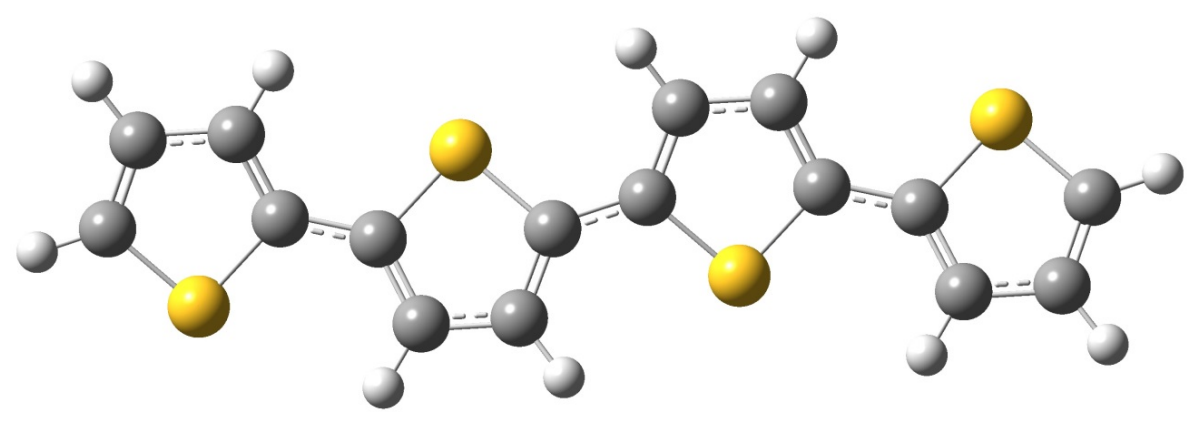

Figure 7.13. Structure used to simulate the Raman spectrum of PQT. This structure is generic for all polythiophenes.

As we did with the Pentacene SERS spectrum, we now try to simulate the SERS by incorporating disordered PQT molecular structures into the DFT calculations. Since the SERS spectrum is detected at the Au-PQT interface where Au molecules may penetrate into the PQT film, we use simple Au-polythiophene structures where lone Au molecules are placed in close proximity to polythiophene monomer units. Two such structures are shown in Figure 7.14. These are the minimal energy configurations of each of the cases where $\mathrm{Au}$ is bonded to polythiophene and $\mathrm{Au}$ is just placed in proximity to polythiophene. The Raman spectra of these structures are shown in Figure 7.15. It is immediately obvious that the addition of Au to the polythiophene monomers, especially in the case where the $\mathrm{Au}$ is bonded to polythiophene, re-creates the enhancement of peaks seen in the SERS spectrum. Specifically, the C-H bend and C-C intra-ring stretch modes are strongly enhanced. 


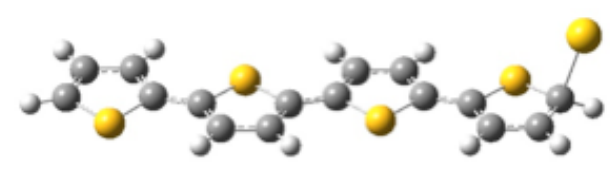

(a)

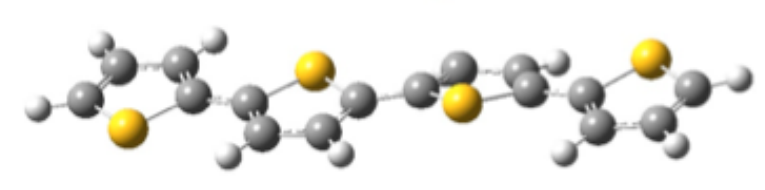

(b)

Figure 7.14. The structures of Au-Polythiophene disordered complexes. (a) a polythiophene subunit with a $\mathrm{Au}$ atom attached at its end (edge $\mathrm{Au}$ ) and (b) a polythiophene subunit with a $\mathrm{Au}$ atom placed at its center (center $\mathrm{Au}$ ). In both cases, the Au atom is $\sim 2 \AA$ from the polythiophene molecule.

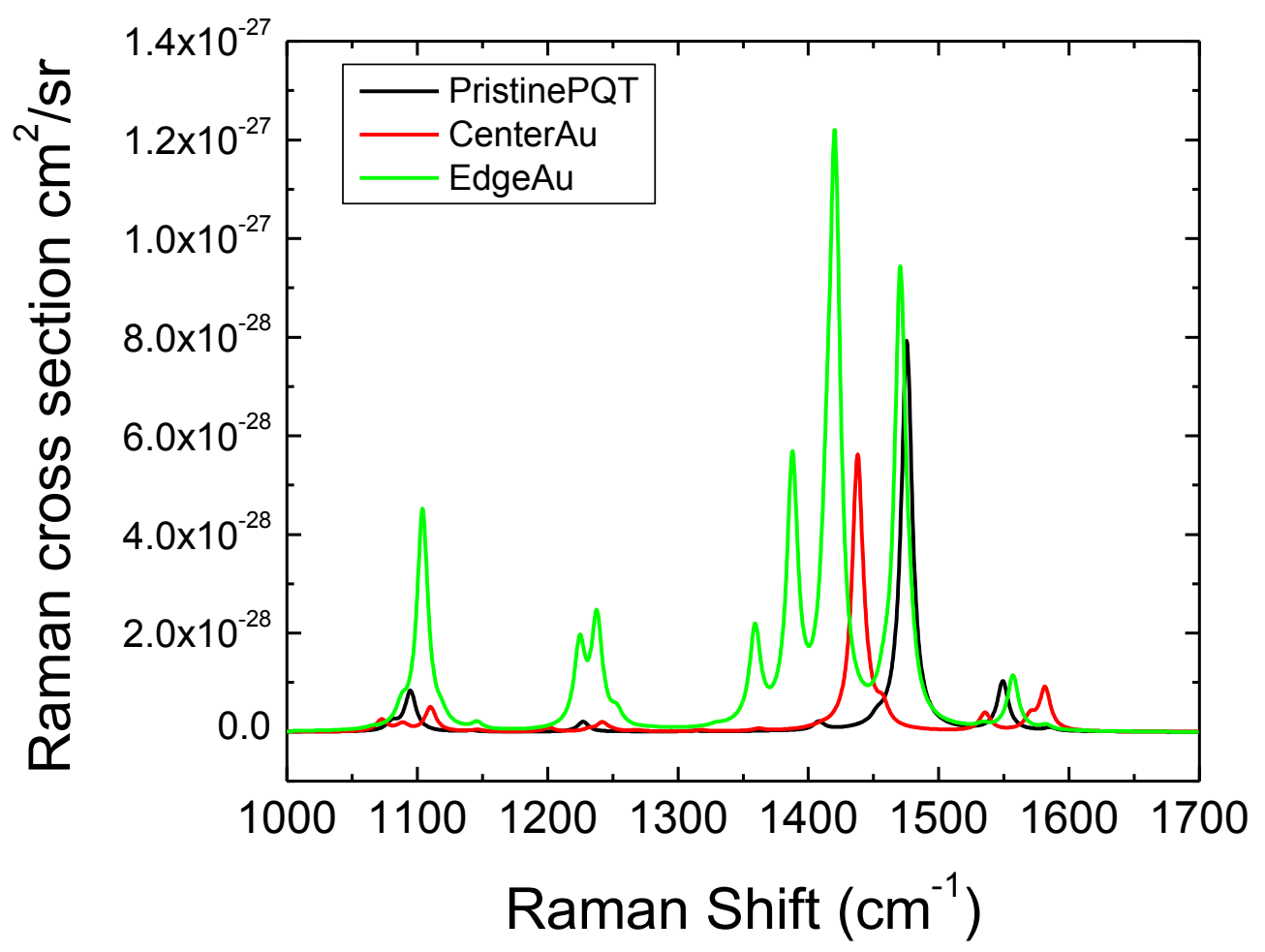

Figure 7.15. The Raman spectra of the PQT-Au structures given in Figure 7.14 
As with Pentacene, the SERS spectrum of PQT is shown to have a high sensitivity to disorder within the PQT film. This disorder is undetectable with regular Raman spectroscopy. Since, in the pentacene OFET system, we were able to demonstrate how this disorder correlates with device performance, it is logical to investigate if a similar analysis can be done here. We note that while both pentacene and PQT are highly conjugated organic systems, they are distinct in several ways. Specifically, while one is a small molecule that must be thermally evaporated in order to form a film, the other is polymer that must be spun-cast. Furthermore, the polythiophene system is susceptible of oxidative degradation in ways the pentacene system isn't. One may ask if the SERS based diagnostic tool developed in chapter 7 may be applied to this system. A preliminary attempt to do this is discussed in appendix C.

In conclusion, The Raman spectrum of a PQT film was found to exhibit the SERS effect when probed in the contact region of an OFET. The SERS spectrum was shown, via DFT computations, to have sensitivity to Au-induced disorder within the film. The OFETs themselves showed a remarkable degradation in performance over time when run in ambient conditions. This is on account of the well-known susceptibility of polythiophene polymers to oxidative degradation. While PQT is designed to minimize this weakness, the PQT polymer likely has some inherent susceptibility. 


\section{Summary and Future Work}

This work was a study of interfaces in OFETs. OFETs have application in the development of low cost and flexible electronic circuits, among other uses, and this work contributes to a better understanding of the nature of the interfaces in such devices. The work may be split into two sections that deal with the two critical interfaces. The first part looks at MAPLE as a method for improved device performance based on a better semiconductor-dielectric interface. In the second section, which constitutes the bulk of the work, we combine Raman spectroscopy with electrical characterization to take advantage of the metal-semiconductor interface to build an SERS based diagnostic tool to characterize disorder in semiconductor films and its effect on device performance.

First, we demonstrated that MAPLE is a viable method for forming organic polymer semiconductor films in the fabrication of OFETs. By taking advantage of the layer-bylayer growth that MAPLE provides, we were able to optimize the semiconductordielectric interface. MAPLE also partially eliminates the need for dielectric surface treatments prior to deposition. OFETs fabricated using MAPLE showed better On/Off rations than those fabricated using spin-coating. As MAPLE has been shown to be a promising candidate for device fabrication, further work is needed in applying the technique to other organic semiconductor systems.

Second, by combining Raman spectroscopy with electrical characterization methods, we created a diagnostic tool for studying the molecular origins of OFET performance degradation. We found that the Raman spectrum of pentacene measured through a $\mathrm{Au}$ contact was dramatically different than the spectrum of pristine pentacene. The spectrum 
featured an enhanced signal with selectively enhanced and shifted peaks. All these features pointed to a surface enhancement of the Raman spectrum by the Au top contact. By carefully examining the growth of this enhanced spectrum as a function of Au contact thickness and taking SEM images of the Au contact surface, we correlated the spectrum with the development of surface roughness at the Au-pentacene interface. We finally confirmed, beyond doubt, that the enhancement was an SERS effect created by the interplay of the near infrared excitation source with the Au surface plasmons by noting that a similar effect could not be seen when the excitation source was changed.

After noting that the SERS spectrum had a signature that was similar to that of disordered graphite, we attempted to simulate the spectrum by using DFT to calculate the Raman spectra of disordered pentacene molecules. A first attempt of incorporating disorder via a simple $\mathrm{sp}^{3}$ carbon breaks in the pentacene structure proved promising. Features seen in the SERS spectrum were re-created by such $\mathrm{sp}^{3}$-disordered structures. This was followed by a more complete treatment of the molecular origins of the disorder where Au-pentacene complexes were used to simulate the SERS spectrum. Peaks seen in the SERS spectrum were accounted for by specific vibrations in the Au-pentacene complexes, further cementing the idea that the SERS spectrum was selectively enhancing the fingerprint of disorder in the pentacene film.

We then harnessed the SERS spectrum to track disorder in the semiconductor film as a function of bias stress. We found that in those devices where there was performance degradation, it was accompanied by an enhancement of the SERS spectrum. On the other hand, in those devices where there was no performance degradation, there was no concurrent enhancement of the SERS spectrum. Thus, the SERS spectrum showed that 
the performance degradation was, at least in part, caused by molecular degradation in the pentacene film.

Next, the SERS spectrum of Pentacene was also shown to be strongly dependent the thickness of the Au layer. A tool that takes advantage of this to measure the thickness of the Au layer via the SERS spectrum was proposed. This SERS effect was also seen with other metals and organic materials, suggesting that such a tool may have wider applications.

Next, we attempted to apply the opto-electronic tool described above to two polymer semiconductor systems. First, two DPP based polymers were used to in the fabrication of OFETs for the first time. The interface properties of PDPP-BBT OFETs were further probed using Raman scattering. Raman linescans across the channel-contact region from devices before and after biasing showed almost no changes indicating that there were no structural changes upon the application of an electric field. Furthermore, the metalpolymer contact seemed to have no impact on the vibrational spectra, unlike pentacene based devices. Second, preliminary attempts were made to simulate the SERS spectrum seen in polythiophene based OFETs. As with pentacene, the SERS spectrum here was shown to detect signs of disorder in the semiconductor film

Since the SERS effect has been seen in multiple systems, it follows that further work is required to see if the SERS based diagnostic tool can be applied more broadly to the field of organic devices. Along with similar experiments with different organic materials, one might also attempt to track the growth of the SERS spectrum in-situ during bias stress. Doing so may give further insight into the origins of molecular disorder in the film. Finally, it is reasonable to expect that the plasmonic states that create the SERS 
effect may also have a direct effect on charge injection or charge conduction at the metalsemiconductor interface. One may attempt to combine laser excitation along with electrical characterization of OFETs to see if the plasmonic effect can be isolated. Measuring and understanding the effect are prerequisites to the final goal of being able to control and use the effect in such devices. 


\section{Appendix A. Effect of oxygen on the Raman spectrum of pentacene}

In order to observe the influence of oxygen on the Raman spectrum of pentacene, we exposed a film of pentacene to a peroxide treatment. A pentacene film grown on a silicon substrate was suspended over a $\sim 10 \mathrm{ml}$ of $30 \% \mathrm{H}_{2} \mathrm{O}_{2}$ in a loosely closed container. A small amount of catalytic silver powder was added to the peroxide to initiate decomposition in order to create an environment with an elevated oxygen concentration. The experimental Raman spectra (normalized to the $1370 \mathrm{~cm}^{-1}$ peak) of pentacene are shown in Fig. A1 (a). The relative intensity of the $1597 \mathrm{~cm}^{-1}$ Raman peak increases for the film exposed to the oxidizing environment, shown by the dotted line. We have further calculated the Raman spectrum of a pentacene molecule with two O-H groups attached to the central ring. Previous work has shown that this molecule is an expected oxidation product of pentacene. ${ }^{114}$ The Raman activity of the $1300-1400 \mathrm{~cm}^{-1}$ region for the hydroxyl pentacene decreases as seen in Fig. A1 (b); the Raman activity of the $1620 \mathrm{~cm}^{-1}$ Raman peak (similar to the experimental $1597 \mathrm{~cm}^{-1}$ peak), however, increases. The calculated spectrum corroborates our experiments where an enhancement of the $\mathrm{C}-\mathrm{C}$ stretch motion at $1597 \mathrm{~cm}^{-1}$ is associated with uptake of oxygen/OH by the molecule.

We point out upon bias stress measurements (which were conducted in air), the Raman spectra do not show an increase in intensity of the $1597 \mathrm{~cm}^{-1}$ peak compared to the pre-bias spectrum. The optical studies indicate more of a distortion of the molecule, which is seen as a signature of disordered $\mathrm{sp}^{2}$ carbons. [One cannot of course rule out the influence of oxygen/water on transport, especially the change in threshold shifts, which is 
related to the trap states. Our optical studies, however, do indicate a structural change of the pentacene molecules.]
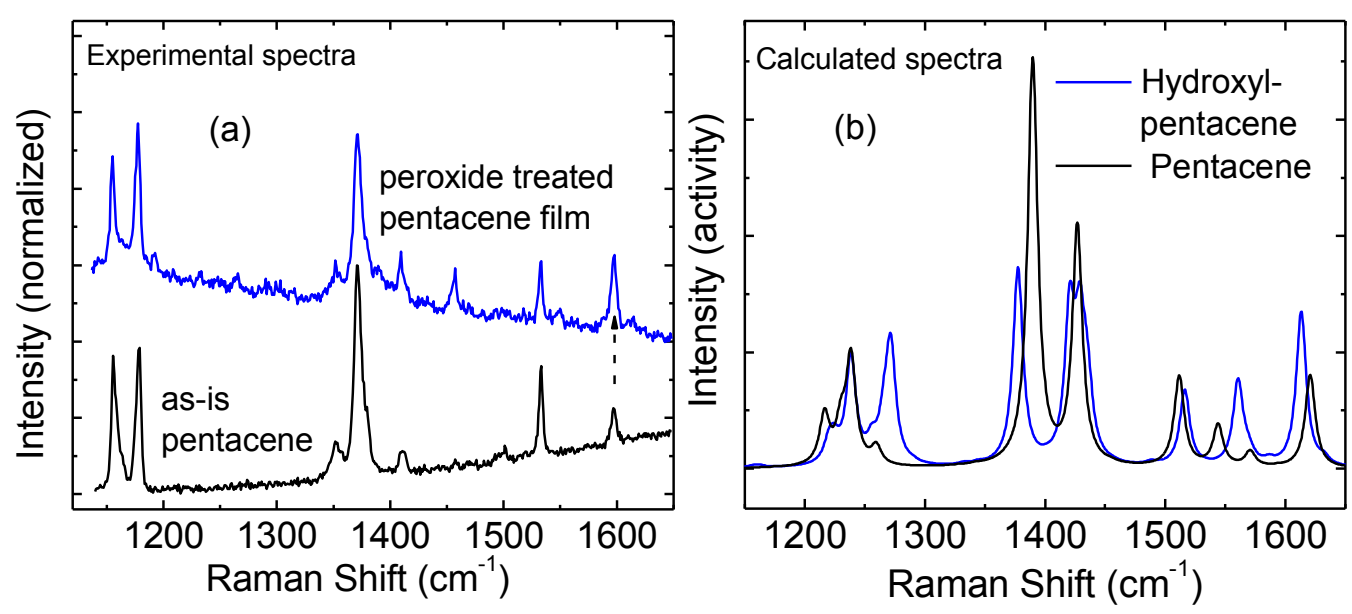

Figure S2. (a) Experimental Raman spectra of as-is pentacene film and a peroxidetreated pentacene film. (b) Calculated Raman spectra of a pristine pentacene molecule and one with two O-H groups attached to the central ring. 


\section{Appendix B. DFT calculations of Herringbone pentacene structures}

The Au-pentacene complexes discussed in chapter 6 were found to reliably recreate the SERS spectrum of pentacene. These structures featured Au atoms placed in close proximity to two pentacene molecules, simulating the interaction of Au atoms, which had penetrated into a pentacene film, with pentacene atoms. However, since pentacene crystalizes in the herringbone pattern, one may ask if simulating the Au-pentacene interaction with the pentacene molecules in this configuration give different results. To investigate this, the Raman spectrum of pristine pentacene and pentacene with Au atoms were computed and compared to the results discussed in chapter six. It was found that the Raman spectra of such structures are more or less identical to the spectra found in chapter six. In fact the Raman spectrum of pristine pentacene in the herringbone pattern was found to be exactly identical that of a single molecule of pentacene, at least in the energy range discussed in this report. Therefore, it is entirely reasonable to use simplified structures to compute Raman frequencies and intensities.

The herringbone Au-pentacene structure used is shown in figure B1. An Au atom has been inserted near the center of the structure. The Raman spectrum of the structure is provided in figure B2. The Raman spectrum of this Au-pentacene complex is very similar to the spectra of the pentacene-Au complexes used in the earlier discussion. 


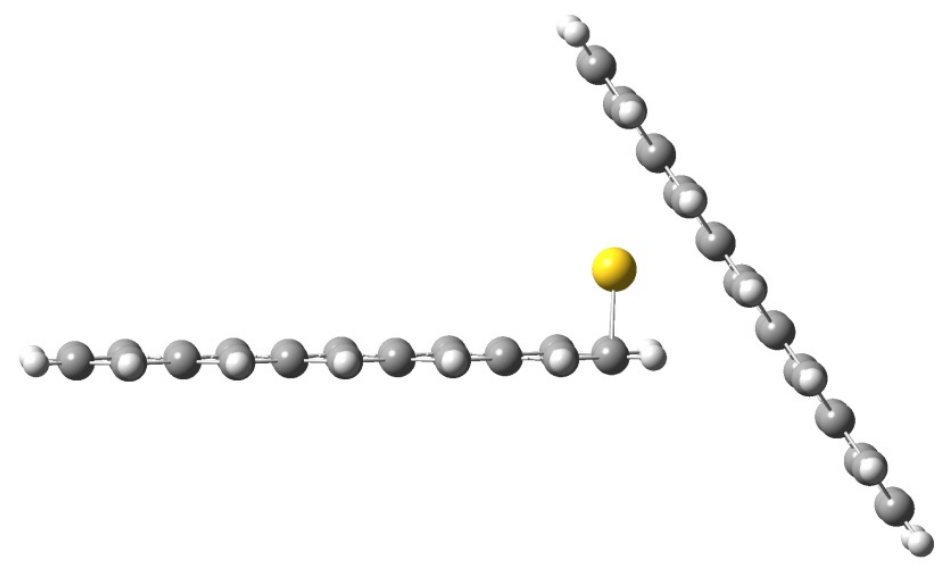

Figure B1. An Au atom combined with two pentacene molecules in the herringbone basis pattern.

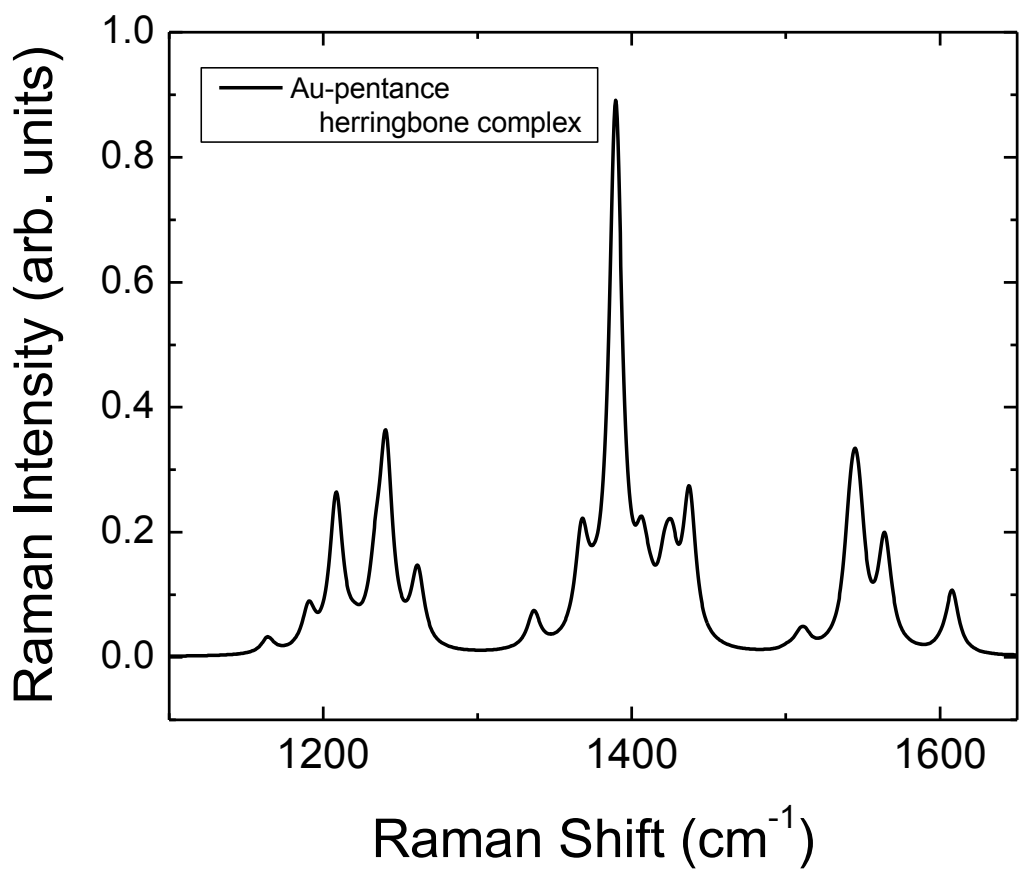

Figure B2. The Raman spectrum of the structure shown in figure B2. 


\section{Appendix C. Applying SERS based diagnostic tool to PQT OFETs}

In chapter 7, it was shown that the SERS spectrum of the semiconductor film in pentacene OFETs detected disorder associated with bias-stress induced degraded performance. Here, we attempt to do a similar analysis with PQT based OFETs. The transfer characteristics of a representative pristine PQT OFET fabricated on an OTS treated substrate are shown in Figure $\mathrm{C} 1$, both in its pristine and biased states. The characteristics were measured in ambient conditions with $\mathrm{V}_{\mathrm{DS}}=-40 \mathrm{~V}$. Device parameters such as charge carrier mobility, threshold voltage, and On/Off ratio were estimated by using the standard current-voltage characteristics of OFETs given by eq. 2.7. The charge carrier mobility of the pristine device was $3.70 \times 10^{-3} \mathrm{~cm}^{2} / \mathrm{Vs}$, the threshold voltage was $1.8 \mathrm{~V}$, and the On/Off ratio was $10^{3}$. The device was then left on in the saturation regime, by setting $\mathrm{V}_{\mathrm{DS}}=\mathrm{V}_{\mathrm{G}}=-40 \mathrm{~V}$, for 20 minutes under ambient conditions. During this time, the drain current of the OFET dropped by $\sim 95 \%$. This remarkable drop in performance is in stark contrast to that of pentacene based devices, where a much smaller deterioration is observed. The transfer characteristics were then re-measured. While the On/Off ratio was found to remain unchanged at $10^{3}$, the charge carrier mobility was found to decrease to $2.80 \times 10^{-3} \mathrm{~cm}^{2} / \mathrm{Vs}$. However, most remarkably, the threshold voltage degraded to $-18.5 \mathrm{~V}$. The device parameters for both the pristine and the biased devices are tabulated in table C.1. 


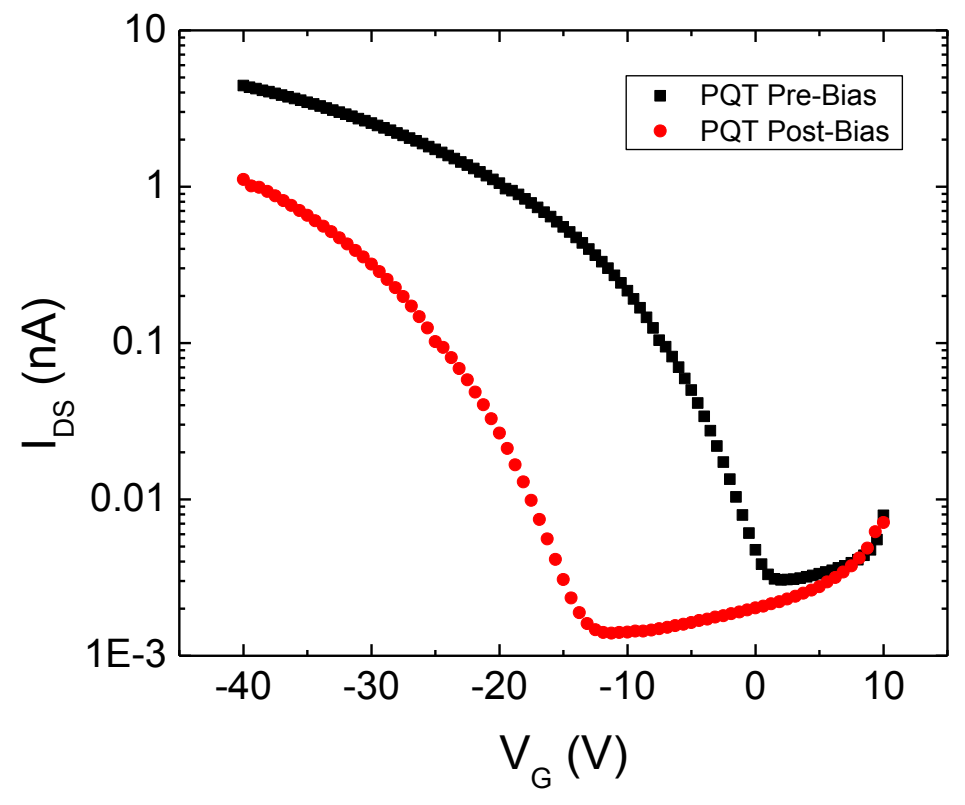

Figure C1. The transfer characteristics of a PQT OFET, both before (pre-bias) and after (post-bias) subjecting the device to a bias stress by leaving it on in the saturation regime for 20 minutes.

Table C.1. Device parameters for a PQT OFET, both before and after bias stress.

\begin{tabular}{lccc}
\hline & $\begin{array}{c}\text { Mobility } \\
\left(\mathbf{c m}^{2} / \mathbf{V s}\right)\end{array}$ & $\begin{array}{c}\text { Threshold } \\
\text { Voltage } \\
(\mathbf{V})\end{array}$ & On/Off ratio \\
\hline Before bias stress & $3.7 \times 10^{-3}$ & -1.8 & $\sim 10^{3}$ \\
After bias stress & $2.8 \times 10^{-4}$ & -18.5 & $\sim 10^{3}$ \\
\hline
\end{tabular}

At this point, it should be emphasized that while PQT is more resistant to oxidative degradation than other polythiophene polymers, it may still have some inherent susceptibility. Perhaps for PQT devices, a large portion of the bias stress induced performance degradation is caused by oxidative degradation rather than electric-field induced degradation, as was found to be the case for pentacene devices. To further 
explore this, the transfer characteristics of the device were re-measured after 3 days. The device was found to be non-functional at this point. Furthermore, other devices that had not been exposed to the same bias stress were also found to be non-functional. This indicates that the precipitous drop in performance in PQT OFETs is, in large part, due to effects distinct from electric field induced degradation.

To test this hypothesis, a Raman map of the PQT OFET taken after the bias stress was compared to one measured before. Each Raman linescan map consisted of $\sim 13$ spectra spanning $60 \mu \mathrm{m}$; the first measurement $(0 \mu \mathrm{m})$ was taken in the channel itself, directly on pentacene, and the last measurement was on the Au pad. The two maps are shown in Figure $\mathrm{C} 2$. Each of the two maps is dominated by the $\mathrm{C}=\mathrm{C}$ stretch peak at $1450 \mathrm{~cm}^{-1}$ and C-C intra-ring stretch peak $1380 \mathrm{~cm}^{-1}$. The intensity of these two peaks is greatest at the edge of the contact region of the OFET. Here, the Au contact is thinnest due to a shadowing effect from the thermal evaporation mask. As was shown in chapter 5, the SERS effect is strongest for thin Au overlayers, so this is expected. Regardless, it is apparent that the SERS signal is stronger from the biased device. The intensity of both the $1450 \mathrm{~cm}^{-1}$ and $1380 \mathrm{~cm}^{-1}$ peaks are greater. This is indicative of greater disorder in the PQT film. As was the case with pentacene-based devices, this indicates that there is electric field induced disorder introduced into the PQT film. However, to fully understand these degradation effects, the bias stress must be done in an inert atmosphere in order to isolate electric field based effect from oxidation based effects. 


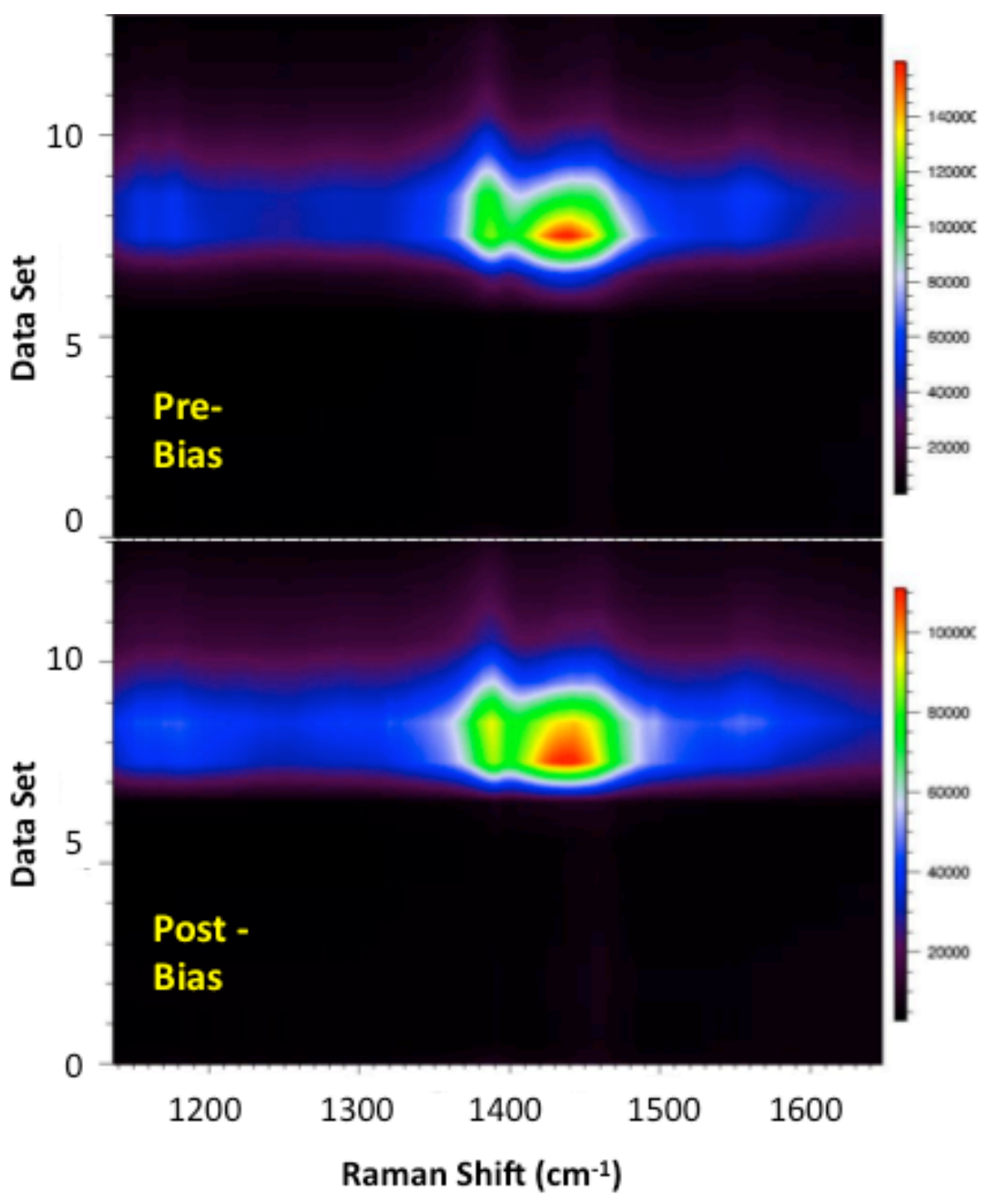

Figure C2. The Raman maps of a PQT OFET, both before (pre-bias) and after (postbias) subjecting the device to a bias stress by leaving it on in the saturation regime for 20 minutes. 


\section{REFERENCES}

1 C. K. Chiang, C. R. Fincher, Y. W. Park, A. J. Heeger, H. Shirakawa, E. J. Louis, S. C. Gau, A. G. MacDiarmid, Phys. Rev. Lett. 39, 1098 (1977).

2 H. Sirringhaus Nature Mater. 2, 641 (2003).

3 V. Coropceanu, J. Cornil, D. A. da Silva Filho, Y. Oliver, R. Silbey, J. L. Bredas, Chem. Rev. 107, 926 (2007)

4 G. Horowitz, Adv. Mater. 10, 365 (1998).

5 M. W. Wu, E. M. Conwell, Chem. Phys. Lett. 266, 363 (1997)

6 G. Horowitz, M. E. Hajiaoui, R. Hajiaoui, J. Apl. Phys. 87, 4456 (2000)

7 A. J. Heeger, S. Kivelson, J. R. Schrieffer, W. P. Su,Rev. Mod. Phys. 60, 781 (1988).

8 A. W. Hofmann, Proc. Royal Soc. 8, 1 (1855).

9 P. Parisse, S. Picozzi, M. Passacantando, L. Ottaviano, Thin Sol. Films 515, 8316 (2007).

10 S. C. B. Mannsfeld, A. Virkar, C. Reese, M. T. Toney, Z. Bao, Adv, Mat. 21, 2294 (2009)

11 H. Yang, T. J. Shin, M. M. Ling, K. Cho, C. Y. Ryu, Z. Bao, J. Am. Chem. Soc. 127, 11542 (2005).

12 D. Knipp, R. A. Street, A. Volkel, J. Ho, J. Appl. Phys. 93, 347 (2003).

13 D. J. Gundlach, Y. Y. Lin, T. N. Jackson, S. F. Nelson, D. G. Schlom, IEEE elec. Dev. Lett. 18, 87 (1997).

14 L. Zhang, S. M. Fakhouri, F. Liu, J. C. Timmuos, N. A. Ran, A. L. Briseno, J. Mater. Chem. 21, 1329 (2011).

15 J. Zaumseil, H. Sirringhaus, 2007, Chem. Rev. 107, 1296 (2007).

16 J. D. Jackson, “Classical Electrodynamics”, Wiley, New York (1998).

17 C. V. Raman, Ind. J. Phys. 2, 387 (1928).

18 A. Singha, P. Dhar, A. Roy, Am. J. Phys. 73, 224 (2005). 
19 J. D. Lorentzen, S. Guha, J. Menéndez, P. Giannozzi, S. Baroni, Chem. Phys, Lett. 270, 129 (1997).

20 M. Moskovits, Rev. Mod. Phys. 57, 783 (1985).

21 K. Kneip, H. Kneipp, I. Itzkan, R. R. Dasari, M. S. Feld, J. Phys.: Condens. Matter. 14, R597 (2002).

22 D. S. Wang and M. Kerker, Phys. Rev. B. 24, 1777 (1981).

23 K. Kneip, H. Kneipp, I. Itzkan, R. R. Dasari, M. S. Feld, Chem. Rev. 99, 2957 (1991).

24 W. Kohn, Rev. Mod. Phys. 71, 1253 (1999).

25 A. P. Sutton, Electronic Structures of Materials; Oxford Press: Oxford: 1993.

26 K. Burke, The ABCs of DFT, http://dft.uci.edu/doc/g1.pdf.

27 GAUSSIAN09, Gaussian, Inc., Pittsburgh, PA, 2009.

28 A. D. Becke, J. Chem. Phys. 98, 5648 (1993).

29 J. S. Binkley, J. A. Pople, W. j. Hehre, J. Am. Chem. Soc, 102, 939 (1980).

30 P J. Hay and W. R. Wadt, J. Chem. Phys. 82, 299 (1985).

31 P J. Hay and W. R. Wadt, J. Chem. Phys. 82, 270 (1984)

32 Y. Yang, M. N. Weaver, and K. M. Merz Jr., J. Phys. Chem. A 113, 9843 (2009).

33 J. Veres, S. Ogier, G. Lloyd, Chem. Mater. 16, 4543 (2004).

34 J. H. W. Smith and I. G. Hill, J. Appl. Phys. 101, 044503 (2007).

35 F. Todescate, R. Capelli, F. Dinelli, M. Muriga, N. Camaioni, M. Yang, R. Bozio, M. Muccini, J. Phys. Chem. B 112, 10130 (2008).

36 L. L. Chua, J. Zamseil, J. F. Chang, E. C. W. Ou, P. K. H. Ho, H. Sirringhaus, R. H. Friend, Nature 434, 194 (2005).

37 S. C. Kim, S. H. Kim, J. H. Lee, M. K.Kim, T. Zyung, Syn. Met. 148, 75 (2005).

38 M .E. McGovern, K. M. R. Kallury, M. Thompson, Langmuir 10, 3607 (1994).

39 P. Silberzan, L. Leger, D. Ausserre, J. J Benattar, Langmuir 7, 1647 (1991).

40 D. L. Angst, G. W. Simmons, Langmuir 7, 2236 (1991). 
41 C. P. Tripp, M. L. Hair, Langmuir 8, 1120 (1992).

42 T. Leitner, G. Friedbacher, T. Vallant, H. Brunner, U. Mayer, H. Hoffman, Mikrochimica Acta 133, 331 (2001).

43 J. B. Brzoska, I. B. Azouz, F. Rondelez, Langmuir 10, 4367 (1994).

44 K. Stowe, Introduction to Statistical Mechanics and Thermodynamics, John Wiley \& Sons, New York (1984).

45 D. R. Lide (Ed.), CRC Handbook of Chemistry and Physics, 84th Ed., CRC Press, Boca Raton, Fl (2003).

46 B. Toftmann, K. Rodrigo, J. Schou, and R. Pedrys, Appl. Surf. Sci. 247, 211 (2005).

47 A. Piqué, R. A. Miquel, D. B. Chrisey, D. Leonhardt, T. E. MsIna, B. J. Spargo, J. H. Callahan, R. W. Vachet, R. Chung, M. A. Bucaro, Thin Sol. Films, 355, 536 (1999).

48 N. B. Ukah, D. Adil, J. Granstrom, R. K. Gupta, K. Ghosh, S. Guha, Organic Elect. 12, 1580 (2011).

49 R.K. Gupta, K. Ghosh, P.K. Kahol, J. Yoon, S. Guha, Appl. Surf. Sci. 254, 7069 (2008).

50 T. Tunno, A.P. Caricato, M.E. Caruso, A. Luches, M. Martino, F. Romano, D. Valerini, and M. Anni, App. Surf. Sci. 253, 6461 (2007).

51 A. Gutierrez-Llorente, G. Horowitz, R. Perez-Casero, J. Perriere, J. L. Fave, A. Yassar, C. Sant, Org. Elect. 5, 29 (2004).

52 A. Di Carlo, F. Piacenza, A. Bolognesi, B. Stadlober, and H. Maresch, Appl. Phys. Lett. 86, 263501 (2005).

53 C. Wang, L. H. Jimison, L. Goris, I. McCulloch, M. Heeney, A. Ziegler, and A. Salleo, Adv. Mat. 22, 697 (2010)

54 K. P. Pernstich, S. Haas, D. Oberhoff, C. Goldmann, D. J. Gundlach, B. Batlohh, A.N. Rashid, and G.Schitter, J. Appl. Phys. 96, 6431 (2004)

55 S. Grecu, M. Bronner, A. Optiz, W. Brutting, Synth. Met. 146, 359 (2004).

56 H. Hasegawa, K. E. Forward, H. Hartmagel, Appl. Phys. Lett. 26, 567 (1975).

57 D. Adil, N. B. Ukah, R. K. Gupta, K. Ghosh, S. Guha, Synthetic Metals 160, 2501 (2010). 
58 G. Salvan, Y. Sakurai, A. Y. Kobitski, R. Scholz, S. Astilean, T. U. Kampen, D. R. T. Zahn, H. Ishii, K. Seki, Appl. Surf. Sci. 190, 371 (2002)

R. J. Davis, J. E.Pemberton, J. Phys. Chem. C 112, 4364 (2008)

60

R. J. Davis, J. E.Pemberton, J.Am. Chem Soc. 113, 10009 (2009); J. Phys. Chem. A 113, 4397 (2009)

61 H. L. Cheng, Y-S. Mai, W. Y. Chou, L. R. Chang, and X. W. Liang. Adv. Funct. Mater. 17, 3639 (2007)

62 H. L. Cheng, W. Y. Chou, C. W. Kuo, Y. W. Wang, Y. S. Mai, F. C. Tang, and S. W. Chu, Adv. Funct. Mater. 18, 285 (2008).

Y. Yamakita, J. Kimura, and K. Ohno, J. Chem. Phys. 126, 064904 (2007).

64 K. Kneipp, W. Y. Wang, H. Kneipp, L. T. Perlman, I. Itzkan, R. R. Dasari, M. S. Feld, Phys. Rev. Lett. 78, 1667 (1997).

M. S. Dresselhaus, G. Dresselhaus, Adv. Phys. 30, 1309 (1981).

66 M. S. Dresselhaus, M. A. Pimenta, P. C. Eklund, G. Dresselhaus, Raman Scattering in Materials Science, Weber, W. H.; Merlin, R. (Eds) 2000, pp. 314.

67 A. Vollmer, H. Weiss, P. Rentenberger, I. Salzmann, J. P. Rabe, N. Koch, Surf. Science 600, 4004 (2006).

J. E. Northrup, M. L .Tiago, S. G. Louie, Phys. Rev. B 66, 121404 (2002).

69 N. J. Watkins and Y. Gao, J. Appl. Phys. 94, 1289 (2003).

70 S. Yoo, B. Domercq, and B. Kippelen, Appl. Phys. Lett. 85, 5427 (2004).

71 P. M. Sanchez, A. J. Cohen, W. Yang, Phys. Rev. Lett. 100, 146401 (2008).

72 N. Abasbegovic, N. Vokutic, L. Colombo, J. Chem. Phys. 41, 2575 (1964).

73 I. Stenger, A. Frigout, D Tondlier, B. Geoffroy, R. Ossikovski, Y. Bonnassieux, Appl. Phys. Lett. 94, 133301 (2009).

74 M. S. Dresselhaus, M. A. Pimenta, P. C. Eklund, G. Dresselhaus, Raman Scattering in Materials Science; W. H. Weber, R. Merlin (Eds.); Springer Series in Materials Science 42; Springer: New York, 2000; 314.

75 H. Sirringhaus, Adv. Mater. 21, 1 (2009).

76 N. B. Ukah, J. Granstrom, R. R. Sanganna Gari, G. M. King, S. Guha, Appl. Phys. Lett. 99, 243302 (2011) 
77 H. L. Cheng, W. S. Mai, W. Y. Chou, and L. R. Chang, Appl. Phys. Lett. 90, 171926 (2007).

78 R. Rosenberg, D. C. Edelstein, C. K. Hu, K. P. Rodbell, Annu. Rev. Mater. Sci. 30, 229 (2000).

79 M. Morgen, E. T. Ryan, J. H. Zhao, C. Hu, T. Cho, P. S. Ho, Annu. Rev. Mater. Sci. 30, 645 (2000).

80 C. J. Brabec, S. Gowrisanker, J. J. M. Halls, D. Laird, S. Jia, S. P. Williams, Adv. Mat. 22, 3839 (2010).

81 W. Shockley, H. J. Queisser, J. Appl. Phys. 32, 510, (1961).

82 H. A. M. Van Mullekom, J. A. J. M. Vekemans, E. E. Havinga, E. W. Meijer, Mat. Sci. and Engin. R 32, 1 (2001).

83 K. Zhang, B. Tieke, Macromolecules, 41, 7287 (2008).

84 M. Tantiwiwiat, A. Tamayo, N. Luu, X. D. Dang, T. Q. Nguyen, J. Phys. Chem. C 112, 17402 (2008).

85 H. Bronstein, Z. Chen, R. S. Ashraf, W. Zhang, J. Du, J. R. Durrant, P. S. Tuladhar, K. Song, S. E. Watkins, Y. Geerts, M. M. Wienk, R. A. J. Janssen, T. Anthopoulos, H. Sirringhaus, M. Heeney, I. McColloch, J. Am. Chem. Soc. 133, 3272 (2011).

86 Y. Li, P. Singh, P. Sonar, Adv. Mat. 22, 4862 (2010).

87 Z. Chen, M. J. Lee, R. S. Ashraf, Y. Gu, S. Albert-Seifried, M. M. Nielsen, B. Schroeder, T. D. Anthopoulos, M. Heeney, I. McColloch, H. Sirringhaus, Adv. Mat. 24, 647 (2012).

88 F. Liu, Y. Gu, C. Wang, W. Zhao, D. Chen, A. L. Briseno, T. P. Russel, Adv. Mat. 24, 3947 (2012).

89 J. Hou, H. Y. Chen, S. Zhang, R. I. Chen, Y. Yang, Y. Wu, G. Li, J. Am. Chem. Soc. 131, 15586 (2009).

90 J. Yuan, X. Huang, F. Zhang, J. Lu, Z. Zhai, C. Di, Z. Jiang, W. Ma, J. Mat. Chem. 22, 22734, (2012).

91 C. Piliego, T. W. Holcombe, J. D. Douglas, C. H. Woo, P. M. Beaujuge, J. M. J. Frechet, J. Am. Chem. Soc., 132, 7595 (2010).

92 C. Kanimozhi, P. Balraju, G. D. Sharma, S. Patil, J. Phys. Chem. B. 114, 3095 (2010); ibid. J. Phys. Chem. C 114, 3287 (2010).

93 A. Dreuw, J. L. Weisman, M. Head-Gordon, J. Chem. Phys. 119, 2943 (2003). 
D. J. Tozer, J. Chem. Phys. 119, 12697, (2003).

95 T. Yanai, D. P. Tew, N. C. Handy, Chem. Phys. Lett. 393, 51 (2004).

96 M. J. G. Peach, T. Helgaker, P. Salek, T. W. Keal, O. B. Lutnaes, D. J. Tozer, N. C. Handy, Phys Chem. Chem. Phys., 8, 558 (2006).

97 Y. Zhao, D. G. Truhlar, J. Phys. Chem A Lett. 110, 13126 (2006).

98 M. P. Marder, “Consendsed Matter Physics”, Wiley, Hoboken, New Jersey (2010).

99 J. F. Chang, B. Sun, D. W. Breiby, M. M. Nielsen, T. I. Solling, M. Giles, I. McCulloch, H. Sirringhaus, 16, 4772 (2004).

100 Kline, R. J.; McGehee, M. D.; Kadnikova, E. N.; Liu, J.; Fréchet, J. M. J. Adv. Mater. 15, 1519 (2003).

101 S. Lunak, J. Vynuchal, P. Horackova, B. Frumarova, Z. Zak, J. Kuceri, O. Salyak, J. Mol. Struct. 39, 983 (2010)

102 C. Volz, M. Arif, S. Guha, J. Chem. Phys. 126, 064905 (2007).

103 S. Guha, W. Graupner, R. Resel, M. Chandrasekhar, H. R. Chandrasekhar, R. Glaser, G. Leising, Phys. Rev. Lett. 82, 3625 (1999).

104 T. Yamamoto, D. Komarudin, M. Arai, B. L. Lee. H. Suganuma, N. Asakawa, Y. Inoue, K. Kubota, S. Sasaki, T. Fukuda, H. Matsuda, J. Am. Chem. Soc. 120, 2047 (1998).

105 K. J. Baeg, D. Khim, D. Y. Kim, J. B. Koo, I. K. You, W. S. Choi, Y. Y. Noh, Thin Sol. Films 518, 4024 (2010).

106 G. Li, V. Shrotriya, J. Huang, Y. Yao, T. Moriarty, K. Emery, Y. Yang, Nature Mater. 4, 864 (2005).

107 M. Reyes-Reyes, K. Kim, D. L. Carrol, Appl. Phys. Lett. 87, 083506 (2005).

108 M. Manceau, A. Rivaton, J. L. Gardette, S. Guillerez, N. Lemaitre, Polym. Degrad. Stab. 94, 898 (2009).

109 Y. Aoyama, T. Yamanari, N. Koumura, H. Tachikawa, M. Nagai, Y. Yoshida, Polym. Degrad. Stab. 98, 899 (2013)

110 B. S. Ong, Y. Wu, P. Liu, S. Gardner, J. Am. Chem. Soc. 1261, 3378 (2004)

111 B. S. Ong, Y. Wu, P. Liu, Proc. of the IEEE 93, 1412 (2005). 
112 W. C. Tsoi, D. T. James, J. S. Kim, P. G. Nicholson, C. E. Murphy, D. D. C. Bradley, J. Nelson, J. S. Kim, J. Am. Chem. Soc. 133, 9834 (2011).

113 B. S. Ong, Y. Wu, Y. Li. P. Liu, H. Pan, Chem Eur. J. 14, 4766, (2008).

114 F. D. Angelis, M. Gaspari, A. Procopio, G. Cuda, E. D. Fabrizio, Chem. Phys. Lett. 193, 468 (2009). 


\section{Publications}

- D. Adil and S. Guha, "Surface-enhanced Raman spectroscopic studies of the Aupentacene interface: a combined experimental and theoretical investigation" J. Chem. Phys. 139, 044715 (2013).

- D. Adil and S. Guha, "Surface-enhanced Raman spectroscopic studies of metalsemiconductor interfaces in organic field effect transistors" J. Phys. Chem. C 116, 12779 (2012).

- N. B. Ukah, D. Adil, R. K. Gupta, K. Ghosh, and S. Guha, "Matrix-assistedpulsed-laser evaporated polymer films in all organic field effect transistors and metal-insulator-semiconductor diodes" Organic Electronics 12, 1580 (2011).

- S. Guha, N. B. Ukah, D. Adil, J. Granstrom, R. K. Gupta, and K. Ghosh, "MAPLE-deposited polymer films for improved organic devices" Applied Physics A 105, 547 (2011)

- D. Adil, C. Kanimohzi, N. B. Ukah, K. Paudel, S. Patil and S. Guha, "Electrical and optical properties of diketopyrrolopyrrole-based copolymer interfaces in thin film devices" ACS Applied Materials and Interfaces 3, 1463 (2011)

- D. Adil, N. B. Ukah, R. K. Gupta, K. Ghosh, and S. Guha "Ineterface-controlled pulsed-laser deposited polymer films in organic devices" Synthetic Metals 160, 2501 (2010) 


\section{Vita}

Danish Adil was born on Janurary $6^{\text {th }}, 1984$ in Chicago, Il. He graduated with a Bachelor's degree in Physics from the Univeristy of Missouri - St. Louis in December 2007. He joined the department of Physics and Astronomy at the University of MissouriColumbia as a graduate student in August 2008. He immediately began working with Dr. Suchi Guha in her research lab. Danish will be graduating with his PhD degree in Physics in the fall of 2013. He will then be moving to Portland, OR, along with his wife, Nida and daughter, Daniya, where he will join Intel Corporation as an engineer. 